\title{
STABILITY OF TRAVELLING WAVE SOLUTIONS OF DIFFUSIVE PREDATOR-PREY SYSTEMS
}

\author{
R. GARDNER AND C. K. R. T. JONES
}

\begin{abstract}
The stability of travelling wave solutions of singularly perturbed, diffusive predator-prey systems is proved by showing that the linearized operator about such a solution has no unstable spectrum and that the translation eigenvalue at $\lambda=0$ is simple. The proof illustrates the application of some recently developed geometric and topological methods for counting eigenvalues.
\end{abstract}

\section{INTRODUCTION}

This paper is concerned with the stability of travelling wave solutions of singularly perturbed systems of reaction-diffusion equations which model predatorprey interactions in mathematical ecology. The waves in question admit a single transition layer outside of which they are slowly varying. In brief, we show that such solutions are stable. This is accomplished by showing that the spectrum of the linearized operator $L$ about the wave intersects a half-plane $\operatorname{Re} \lambda \geq \beta$, for some $\beta<0$, only at $\lambda=0$ and, furthermore, that the translation eigenvalue at $\lambda=0$ is simple. It is well known that linearized stability implies nonlinear stability for this class of equations (see Henry $[\mathrm{H}]$ ).

In a companion paper by Alexander, Gardner, and Jones (see [AGJ]) some new tools for analyzing the spectrum of $L$ were introduced in a general setting. The theory in [AGJ] introduces a certain complex vector bundle $\mathscr{E}$ over a real 2 -sphere which contains information about the number of eigenvalues of $L$ inside a given simple, closed curve $K \subset \mathbb{C}$ not intersecting the spectrum itself. In particular, the main theorem in [AGJ] equates the number of eigenvalues of $L$ inside $K$ (counting multiplicity) with a certain topological invariant, $c_{1}(\mathscr{E})$ of $\mathscr{E}$ called the first Chern number of $\mathscr{E}$. The construction of $\mathscr{E}$ and the characterization of its first Chern number use only elementary aspects of the theory of vector bundles and their characteristic classes and should be readily accessible to analysts and applied mathematicians. The main point is that solutions of the linearized equations which are candidates for eigenfunctions, namely those that

Received by the editors November 21, 1988 and, in revised form, August 14, 1989.

1980 Mathematics Subject Classification (1985 Revision). Primary 35K55.

The first author's research was partially supported by NSF grants DMS-8320485 and DMS8802468 .

The second author's research was partially supported by NSF grants DMS-8501961 and DMS8801627. 
decay to zero at $-\infty$, span vector spaces which are parametrized by the wave itself and by the eigenvalue parameter $\lambda$. Another way of saying this is that they form a vector bundle over this parameter space. It turns out that the twisting of these vector spaces when $\lambda$ is restricted to $K$ determines the number of eigenvalues of $L$ inside $K$. The bundle $\mathscr{E}$ is constructed in a natural way so that its first Chern number measures the twisting of these vector spaces. The full theory is developed in [AGJ]; however, in order to make this paper more self-contained, a brief account of the relevant aspects of the theory in [AGJ] has been provided in $\S$ IIIE and IIIF, together with an outline of the manner in which these tools are employed here.

The theory in [AGJ] is a natural generalization of earlier work of Jones [J] on the stability of the pulse solution of the FitzHugh-Nagumo system. An important observation in the latter part is that the linearized equations (say on $\mathbb{C}^{2 n}$ ) induce a flow on the space of complex lines in $\mathbb{C}^{2 n}$. This flow is obtained by forming the complex span of a given nontrivial solution of the linearized equations. The space of complex lines in $\mathbb{C}^{2 n}$ is called complex projective space and is denoted by $\mathbb{C} P^{2 n-1}$. The linearized equations then induce a flow on this compact manifold; in a given set of local coordinates on $\mathbb{C} P^{2 n-1}$, this gives rise to an associated system of equations which are called the projectivized system. A solution of these equations therefore determines a family of complex lines parametrized by $\lambda$ and by the wave, that is, a complex line bundle over that parameter space. This is the main tool used in obtaining information about $\mathscr{E}$, since certain distinguished solutions of the projectivized system give rise to one-dimensional subbundles $\mathscr{E}_{i}$ of $\mathscr{E}$. In general, if the (complex) dimension of $\mathscr{E}$ is $k$, then there is frequently a way to decompose $\mathscr{E}$ into a direct sum $\mathscr{E}=\bigoplus_{i=1}^{k} \mathscr{E}_{i}$ of one-dimensional subbundles $\mathscr{E}_{i}$ of $\mathscr{E}$. Such a decomposition is called a Whitney sum. The existence of such a decomposition contains global information about the fibers of $\mathscr{E}$. This information is obtained through a characterization of the global behavior of the distinguished solutions of the projectivized system. In particular, the solutions in question are connecting orbits between certain pairs of critical points.

One aspect of this approach is that it reveals how the underlying wave, for which good estimates are readily available, encodes information into the linearized problem. In this setting, the production of eigenvalues can be understood as an essentially nonlinear phenomenon. In the problem in [J], the eigenvalues are determined by the global behavior of one particular solution of the projectivized equations. Its global behavior can be characterized by observing, when a parameter $\varepsilon$ in the equations is small, that the solution remains near an attractor for the reduced projectivized flow (i.e., when $\varepsilon=0$ ). There is an analogous solution in the problem studied here and a similar treatment can be provided (see the "elephant trunk lemma" in $\S I V$ ); indeed, this seems to be a general feature of fast-slow systems. The main difference between the equations in $[\mathrm{J}]$ and those under discussion here is that in the present case, eigenvalues can 
also be produced through the behavior of a second solution of the projectivized linearized system. The global behavior of this second solution is fundamentally different from that of the first solution mentioned above in that it forms a saddle-saddle connection on the projective space. The elephant trunk lemma is therefore not available and different methods for characterizing its behavior need to be devised. The main feature of the equations which make this possible is again the presence of the small parameter $\varepsilon$. Hence both solutions are studied by referring to certain reduced systems when $\varepsilon=0$; for the first, or the fast unstable bundle, these are the stretched equations in the transition layer, while for the second, or slow unstable bundle, these are reduced equations in the slow manifolds. The analysis of the global behavior of these two solutions is contained in $\S \S I V$ and V. These sections are technically involved and could easily be skimmed by readers seeking to first obtain an impressionistic overview.

In the terminology of [AGJ], the unstable bundle $\mathscr{E}$ formed from the linearized equations is 2-dimensional and the fast-slow structure induces a natural splitting of $\mathscr{E}$ into the Whitney sum $\mathscr{E}_{1} \oplus \mathscr{E}_{2}$ of two complex line bundles, wherein $\mathscr{E}_{1}$ is "governed" by the fast reduced system and $\mathscr{E}_{2}$ is "governed" by the slow reduced system. The first Chern number, $c_{1}(\mathscr{E})$, which measures the number of eigenvalues $\lambda$ in the region $\operatorname{Re} \lambda \geq \beta$, can be expressed as $c_{1}\left(\mathscr{E}_{1}\right)+c_{1}\left(\mathscr{E}_{2}\right)$. The proof is completed by showing that $c_{1}\left(\mathscr{E}_{1}\right)=1$ while $c_{1}\left(\mathscr{E}_{2}\right)=0$.

One of our principal objectives here is to illustrate how the machinery in [AGJ] has a direct bearing on singular perturbation problem. In particular, analytical statements concerning the behavior of solutions of the linearized equations are reformulated as geometric statements concerning the behavior of certain associated vector bundles. This is more than a convenient formalism in that the limiting behavior of the geometric entity, the vector bundle, has a clean and natural characterization as the small parameter $\varepsilon$ tends to zero, whereas the associated analytical entities, i.e., solutions of the linearized equations, become singular as $\varepsilon \rightarrow 0$ and it is awkward to characterize their limiting behavior.

Stability theorems for various types of layered solutions of a somewhat similar class of systems have recently been proved by Fujii and Nishiura (see [NF]), which are obtained by a completely different, analytical technique (the SLEP method). In contrasting the two approaches it is apparent that the SLEP method yields finer information about the location of critical eigenvalues, i.e., those tending to zero in the singular limit, than our geometric approach. On the other hand, the SLEP method appears to depend in an essential manner on the system being a two component reaction-diffusion system with small diffusion in one component. The theory in [AGJ] applies to systems with an arbitrary number of components; it is also potentially applicable to systems which are not of reaction-diffusion type. Although some type of fast-slow structure will probably be an essential feature of tractable examples, it is anticipated that the theory in [AGJ] will find applications in other classes of equations, such as conservation laws. 
Finally, we remark that the existence of travelling wave solutions of predatorprey systems has been studied by Gardner (see [G1, G2]).

We conclude this section with a description of the organization of the remainder of the paper.

II. The reaction-diffusion equations and their associated travelling wave systems are formulated, and the main results of the paper are stated. The singular limit at $\varepsilon=0$ is defined and is used to formulate an a priori estimate for the wave when $\varepsilon \ll 1$.

III. The slow and fast linearized equations and the spectra of their asymptotic systems are characterized. The unstable bundle $\varphi^{-}$is defined and an outline of the proof is given. The fast and slow reduced systems are defined.

IV. The elephant trunk lemma is proved and is used to describe the global behavior of the fast unstable subbundle $\hat{e}_{1}$ in $\varphi^{-}$.

V. The slow unstable subbundle $\hat{e}_{2}$ in $\varphi^{-}$is defined. A theorem which characterizes the global behavior of $\hat{e}_{2}$ for small $\varepsilon$ is proved.

VI. The bundle $\mathscr{E}$ is characterized as the Whitney sum of two 1-plane bundles $\mathscr{E}_{1}$ and $\mathscr{E}_{2}$. The Chern number computation is continued to the reduced bundles, $\mathscr{E}_{1 R}$ and $\mathscr{E}_{2 R}$ at $\varepsilon=0$, and $c_{1}$ of $\mathscr{E}_{1 R}$ and $\mathscr{E}_{2 R}$ is computed.

\section{THE MODEL EQUATIONS AND EXISTENCE OF TRAVELLING WAVES}

A. A reaction-diffusion system. The equations to be considered are

$$
u_{1_{t}}=\varepsilon^{2} u_{1 x x}+u_{1} f_{1}(u), \quad u_{2_{t}}=u_{2 x x}+u_{2} f_{2}(u),
$$

where $u=\left(u_{1}, u_{2}\right), x \in \mathbb{R}^{1}$, and $0<\varepsilon \ll 1$ is a small parameter. Here, $u_{2}$ is the population density of a predator species and $u_{1}$ is that of its prey. The nature of the interaction is characterized by the following hypothesis:

$$
\begin{aligned}
& \frac{\partial f_{1}}{\partial u_{2}}<0 \text { and } \frac{\partial f_{2}}{\partial u_{1}}>0 \text {, and the null sets, } f_{1}=0 \text { and } f_{2}=0, \\
& \text { are qualitatively as depicted in Figure } 2.1 \text {. }
\end{aligned}
$$

The equations admit four constant solutions, two of which are stable solutions of (2.1). These are the rest point $\mathscr{O}$ at the origin and the rest point $C=\left(c_{1}, c_{2}\right)$ interior to the positive quadrant. The travelling waves considered here connect $\mathscr{O}$ at $-\infty$ to $C$ at $+\infty$.

Remark. The stability of $\mathscr{O}$ and $C$ relative to (2.1) for small $\varepsilon$ is an immediate consequence of Lemma 3.1. This lemma implies that the spectra of the constant coefficient operators obtained by linearizing about $\mathscr{O}$ and $C$ lie in the region $\operatorname{Re} \lambda<\beta$ for some $\beta<0$.

B. Travelling waves and the statement of the main theorems. Due to the manner in which the small parameter $\varepsilon$ enters into the equations, it is appropriate to look for slow travelling waves. Hence we look for solutions to $(2.1)$ which depend on the single variable

$$
\zeta=x-\varepsilon \theta t,
$$




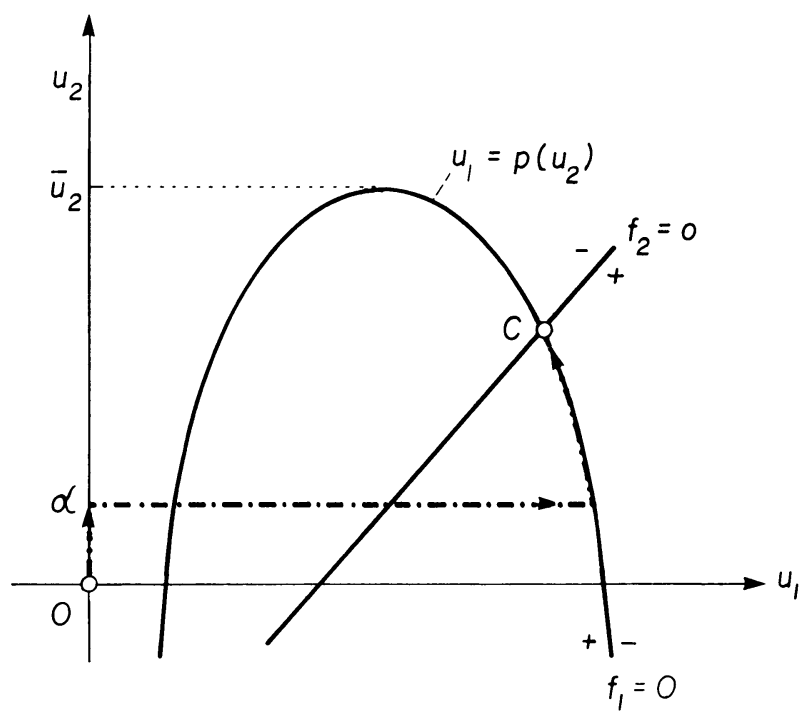

FIGURE 2.1

where $\theta=\theta(\varepsilon)$ remains bounded as $\varepsilon \rightarrow 0$ and $\varepsilon \theta$ is the wave velocity. This ansatz leads to a system of four first order o.d.e.s, which we call the slow travelling wave equations,

$$
\begin{aligned}
\varepsilon \dot{U}_{1} & =V_{1}, \\
\varepsilon \dot{V}_{1} & =-\theta V_{1}-U_{1} f_{1}(U), \\
\dot{U}_{2} & =V_{2}, \\
\dot{V}_{2} & =-\varepsilon \theta V_{2}-U_{2} f_{2}(U),
\end{aligned}
$$

where $U=\left(U_{1}, U_{2}\right)$ and "dot" is $d / d \zeta$. The rest points of $(2.2)_{s}$ are the rest points of $\left(u_{1} f_{1}, u_{2} f_{2}\right)$ augmented with zeros for the $V$-components. With a slight abuse of notation we denote by $\mathscr{O}$ and $C$ the rest points of $(2.2)_{s}$ associated with the rest points $\mathscr{O}$ and $C$ of $(2.1)$.

This paper is concerned with the heteroclinic solutions of $\left(2.2_{s}\right)$ which connect $\mathscr{O}$ at $\zeta=-\infty$ to $C$ at $\zeta=+\infty$. Such solutions are saddle-saddle connections, and the wave will only exist at distinguished values of the parameter $\theta ; \theta$ should therefore be regarded as part of the solution.

The following existence theorem was proved by Gardner in [G2].

Theorem E. Suppose that the vector field $\left(u_{1} f_{1}, u_{2} f_{2}\right)$ satisfies hypothesis $(\mathrm{H} 1)$. Then for sufficiently small $\varepsilon>0$ there exists a travelling wave solution of $(2.2)_{s}$ which connects $\mathscr{O}$ at $\zeta=-\infty$ to $C$ at $\zeta=+\infty$.

This result extends a previous existence theorem (see [G1]) to the singular perturbation regime $\varepsilon \ll 1$. The latter result did not require $\varepsilon$ to be small; however, it imposed more stringent hypotheses on the vector field. Together, the results in [G1, G2] imply that the solutions considered here lie on a branch which continues globally. 
The following theorem is the main result of this paper.

Stability Theorem. Let $A_{i, j}(u)$ denote the entries of the Jacobian matrix of $\left(u_{1} f_{1}, u_{2} f_{2}\right)$ at $u$ and let $Z_{R}$ denote the points in the right-hand branch of $f_{1}=0, u_{1}=p\left(u_{2}\right)$ (see Figure 2.1), for which $u_{2} \geq 0$. Suppose in addition to the hypotheses of Theorem $\mathrm{E}$, that

$$
\max _{u \in Z_{R}} \frac{\left|A_{12}(u) A_{21}(u)\right|}{A_{11}(u)^{2}}<1 .
$$

Then for sufficiently small $\varepsilon>0$ the travelling waves of Theorem $\mathrm{E}$ are stable solutions of (2.1). More precisely, every solution of (2.1) whose data are uniformly near the u-components of the wave tend to a translate of the wave at an exponential rate as $t \rightarrow+\infty$.

Remark. Hypothesis ( $\mathrm{H} 2)$ will be satisfied if the right branch of $f_{1}=0$ is not too far from the vertical line through $C$. It is quite likely that the travelling wave becomes unstable in the absence of such a condition. It should be noted that $(\mathrm{H} 2)$ is different from the condition used by Fujii and Nishiura to prove stability for a related class of systems.

C. Fast and slow regimes: notation. Solutions of $(2.2)_{s}$ exhibit two distinct time scales. In the slow regime $V_{1}$ remains $\mathscr{O}(1)$ for small $\varepsilon$. Geometrically, this forces bounded solution of $(2.2)_{s}$ to lie near the slow manifolds, which we define as follows. We shall be interested in solutions which, when $\varepsilon=0$, jump from the left stable branch, $u_{1}=0$, to the right stable branch $Z_{R}$ at $f_{1}=0$. The latter can be parameterized by $u_{2}$. Let

$$
x=\left(U_{1}, V_{1}, U_{2}, V_{2}\right)^{t}
$$

denote the vector of dependent variables in (2.2)s. The left and right slow manifolds are defined respectively, as

$$
\begin{gathered}
S_{L}=\left\{X \in \mathbb{R}^{4}: U_{1}=V_{1}=0\right\} \\
S_{R}=\left\{X \in \mathbb{R}^{4}: U_{1}=p\left(U_{2}\right), U_{2} \leq \bar{u}_{2}, \text { and } V_{1}=0\right\} .
\end{gathered}
$$

We shall look for solutions which lie near $S_{L}$ for $\zeta<0$ and near $S_{R}$ for $\zeta>0$.

In order to describe the behavior in the transition layer near $\zeta=0$ we change time scales and introduce stretched variables as follows:

$$
\xi=\zeta / \varepsilon, \quad u_{i}(\xi)=U_{i}(\varepsilon \xi), \quad v_{i}(\xi)=V_{i}(\varepsilon \xi), \quad i=1,2 .
$$

The vector of dependent variables in this scaling is denoted by

$$
x=\left(u_{1}, v_{1}, u_{2}, v_{2}\right)^{t}
$$


and the travelling wave equations assume the form

$$
\begin{aligned}
& u_{1}^{\prime}=v_{1}, \\
& v_{1}^{\prime}=-\theta v_{1}-u_{1} f_{1}(u), \\
& u_{2}^{\prime}=\varepsilon v_{2}, \\
& v_{2}^{\prime}=\varepsilon\left[-\theta \varepsilon v_{2}-u_{2} f_{2}(u)\right],
\end{aligned}
$$

where "prime" is $d / d \xi$. We shall refer to $(2.2)_{f}$ as the fast travelling wave equations.

Throughout the remainder of the paper we shall adhere to the following convention concerning notation: a lower case dependent variable will always represent the fast scaling and will be regarded as a function of $\xi$ while an upper case variable will always represent the slow scaling and will be regarded as a function of $\zeta$.

Finally, it will be convenient to express $(2.2)_{s}$ and $(2.2)_{f}$ in vector form. To this end let $N(X, \theta, \varepsilon)$ be the vector field on the right side of $(2.2)_{s}$ after the first two equations are multiplied by $\varepsilon^{-1}$, and let $n(x, \theta, \varepsilon)$ denote the vector field on the right side of $(2.2)_{f}$. These systems can then be expressed as

$$
\begin{gathered}
\dot{X}=N(X, \theta, \varepsilon), \\
x^{\prime}=n(x, \theta, \varepsilon) .
\end{gathered}
$$

D. Singular limits. In estimating solutions of the linearized equations we shall need precise estimates for the location of the travelling wave for small $\varepsilon$. To this end we set $\varepsilon=0$ in $(2.2)_{s}$. The first two equations require $V_{1}=U_{1} f_{1}(U)=0$, and for the solutions studied here, either $U_{1}=0$ or $U_{1}=p\left(U_{2}\right)$. In the slow manifolds $S_{L}$ and $S_{R}$ the nonlinear functions are therefore

$$
g_{-}\left(U_{2}\right)=U_{2} f_{2}\left(0, U_{2}\right), \quad g_{+}\left(U_{2}\right)=U_{2} f_{2}\left(p\left(U_{2}\right), U_{2}\right) .
$$

The slow reduced equations are therefore

$$
(2.3)_{s R}^{ \pm} \quad \dot{U}_{2}=V_{2}, \quad \dot{V}_{2}=-g_{ \pm}\left(U_{2}\right) \text {; }
$$

it is easily verified that the phase planes for these systems are as depicted in Figures $2.2 \mathrm{a}^{-}, \mathrm{a}^{+}$. We focus on solutions which remain in the $(-)$plane until $U_{2}$ assumes some value $U_{2}=\alpha$ (to be determined), at which point the reduced solution is required to jump to a solution curve in the $(+)$ plane using as data the limiting values of the solution curve in the (-) plane. The slow singular limit is defined as a solution obtained in this manner which connects the origin at $\zeta=-\infty$ to the rest point $\left(c_{2}, 0\right)$ at $\zeta=+\infty$. Since the unstable manifold $\mathrm{M}^{-}$of the origin in the $(-)$plane and the stable manifold $\mathrm{M}^{+}$ of $\left(c_{2}, 0\right)$ in the $(+)$ plane define $V_{2}$ as monotone increasing and monotone decreasing functions of $U_{2}$, respectively, it follows that the value $u_{2}=\alpha$ where the transition occurs is uniquely determined. Each of the systems $(2.3)_{s R}^{ \pm}$can be embedded in $\mathbb{R}^{4}$ by taking $U_{1}=p\left(U_{2}\right)$ and $V_{1}=0$. Let $X_{R}(\zeta)$ denote the 


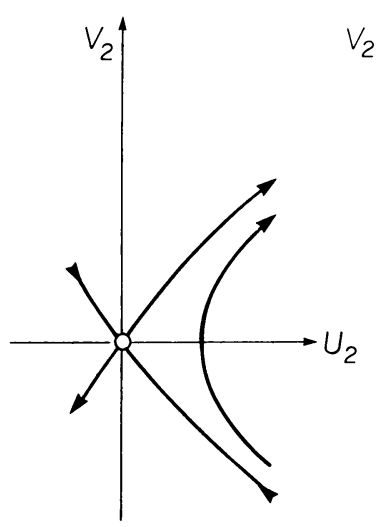

$\left(a^{-}\right)$

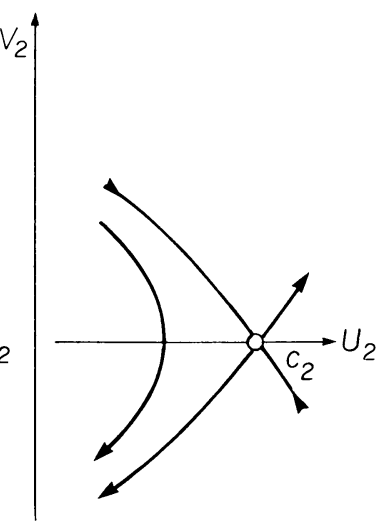

$\left(\mathrm{a}^{+}\right)$

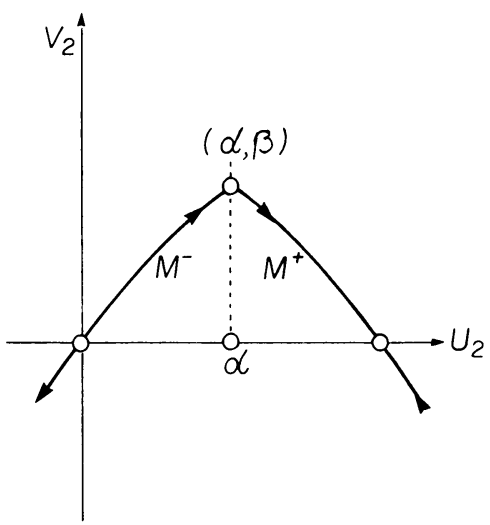

(b)

FIGURE 2.2

solution associated with the solution of $(2.3)_{s R}^{ \pm}$depicted in Figure 2.2(b); we will refer to $X_{R}(\zeta)$ as the slow singular limit. We set the parametrization so that the discontinuity occurs at $s=0$.

The limit $\theta^{*}$ of $\theta$ is $\varepsilon \rightarrow 0$ and the behavior inside the transition layer are determined by the fast reduced system,

$$
\begin{aligned}
& u_{1}^{\prime}=v_{1}, \\
& v_{1}^{\prime}=-\theta v_{1}-u_{1} f_{1}\left(u_{1}, u_{2}\right), \quad \text { or } \quad x^{\prime}=n(x, \theta, 0), \\
& u_{2}^{\prime}=0 \\
& v_{2}^{\prime}=0 .
\end{aligned}
$$

For constant $u_{2}$ with $0 \leq u_{2} \leq c_{2}, u_{1} f_{1}\left(u_{1}, u_{2}\right)$ is a qualitative cubic and the 1-components of $(2.3)_{f R}$ are therefore the travelling wave equations of the bistable, scalar reaction-diffusion equation. It is well known that for each $u_{2}$ there exists a unique wave speed $\theta=\Theta\left(u_{2}\right)$ and a solution $x_{R}\left(\xi, u_{2}\right)$ of $(2.3)_{f R}$ which satisfies

$$
\begin{aligned}
x_{R}\left(\xi, u_{2}\right) & =\left(u_{R}(\xi), v_{R}(\xi), u_{2}, 0\right)^{t}, \\
x_{R}\left(-\infty, u_{2}\right) & =\left(0,0, u_{2}, 0\right)^{t}, \\
x_{R}\left(+\infty, u_{2}\right) & =\left(p\left(u_{2}\right), 0, u_{2}, 0\right)^{t} .
\end{aligned}
$$

(See e.g. Fife $[\mathrm{F}]$.) From the preceding analysis of the slow system we must have that the singular limit in the transition layer is $x_{R}(\xi)=x_{R}(\xi, \alpha)$ and the limit of $\theta$ as $\varepsilon \rightarrow 0$ is $\theta^{*}=\Theta(\alpha)$.

E. A priori estimates. In this section we state an estimate for the travelling wave which is valid for $\varepsilon \ll 1$. The proof can be found in [G2]; see also [GS] for 
related results on periodic waves. This plays a crucial role in understanding how the wave encodes information into the linearized equations.

Consider the curves in $\mathbb{R}^{4}$,

$$
W_{-}=\left\{X_{R}(\zeta): \zeta<0\right\}, \quad W_{F}=\left\{x_{R}(\xi): \xi \in \mathbb{R}^{1}\right\}, \quad W_{+}=\left\{X_{R}(\zeta): \zeta>0\right\},
$$

determined by the slow and fast singular limits at $\varepsilon=0$. Given $\delta>0$, set

$$
\begin{aligned}
n_{ \pm}(\delta) & =\left\{x \in \mathbb{R}^{4}: \operatorname{dist}\left(x, W_{ \pm}\right) \leq \delta\right\}, \\
n_{F}(\delta) & =\left\{x \in \mathbb{R}^{4}: \operatorname{dist}\left(x, W_{F}\right) \leq \delta\right\}, \\
I(\delta) & =\left\{\theta \in \mathbb{R}^{1}:\left|\theta-\theta^{*}\right| \leq \delta\right\},
\end{aligned}
$$

and define

$$
n(\delta)=\left[n_{-}(\delta) \cup n_{F}(\delta) \cup n_{+}(\delta)\right] \times I(\delta) .
$$

Hence $n(\delta)$ is a $\delta$-neighborhood of the singular limit.

Lemma 2.1. Given $\delta>0$ there exists $\bar{\varepsilon} \leq \varepsilon_{0}$ such that

$$
(X(\zeta, \varepsilon), \theta(\varepsilon)) \in n(\delta)
$$

for all $\varepsilon \leq \bar{\varepsilon}$, where $X(\zeta, \varepsilon), \theta(\varepsilon)$ is the travelling wave solution of $(2.2)_{s}$ of Theorem $\mathrm{E}$.

The following result is an immediate consequence of Lemma 2.1 and Gronwall's inequality.

Corollary 2.2. Let $X(\zeta, \varepsilon)=x(\xi, \varepsilon), \theta(\varepsilon)$ be the travelling wave solution of $(2.2)_{s}$. The following are true for each fixed $a>0$ :

$$
\begin{aligned}
& \lim _{\varepsilon \rightarrow 0} x(\xi, \varepsilon)=x_{R}(\xi) \text { uniformly for }|\xi| \leq a, \\
& \lim _{\varepsilon \rightarrow 0} X(\zeta, \varepsilon)=X_{R}(\zeta) \text { uniformly for }|\zeta| \geq a \text {. }
\end{aligned}
$$

\section{THE LINEARIZED EQUATIONS AND ASSOCIATED VECTOR BUNDLES}

A. The equations. Let $X(\zeta, \varepsilon), \theta(\varepsilon)$ denote a travelling wave solution of $(2.2)_{s}$. Henceforth we shall write $\theta$ in place of $\theta(\varepsilon)$. If $U(\zeta, \varepsilon)$ are the $u$-components of $X$, then $U$ is a stationary solution of

$$
\begin{aligned}
& u_{1_{t}}=\varepsilon^{2} u_{1 \zeta \zeta}+\varepsilon \theta u_{1 \zeta}+u_{1} f_{1}(u), \\
& u_{2_{t}}=u_{2 \zeta \zeta} k+\varepsilon \theta u_{2 \zeta}+u_{2} f_{2}(u) .
\end{aligned}
$$

The perturbation equations for solutions $u$ of (3.1) of the form

$$
u(\zeta, t)=U(\zeta, \varepsilon)+\delta e^{\lambda t} P(\zeta)+\mathscr{O}\left(\delta^{2}\right)
$$

yield an eigenvalue problem for $P(\zeta)$ and $\lambda$ :

$$
L(\varepsilon) P \stackrel{\text { def }}{=}\left(\begin{array}{c}
\varepsilon^{2} \ddot{P}_{1}+\varepsilon \theta \dot{P}_{1}+A_{11}(\zeta, \varepsilon) P_{1}+A_{12}(\zeta, \varepsilon) P_{2} \\
\ddot{P}_{2}+\varepsilon \theta \dot{P}_{2}+A_{21}(\zeta, \varepsilon) P_{1}+A_{22}(\zeta, \varepsilon) P_{2}
\end{array}\right)=\lambda P
$$


here

$$
A_{i j}(\zeta, \varepsilon)=\frac{\partial}{\partial u_{j}}\left(u_{i} f_{i}(u)\right) \quad \text { at } u=U(\zeta, \varepsilon) \text {. }
$$

We regard $L(\varepsilon)$ as a densely defined operator on the space $\mathrm{BC}\left(\mathbb{R}^{1}\right)^{2}$ of pairs of uniformly bounded continuous functions on $\mathbb{R}^{1}$.

It is desirable to express (3.2) as the system of first order equations:

$$
\begin{aligned}
\varepsilon \dot{P}_{1} & =Q_{1} \\
\varepsilon \dot{Q}_{1} & =-\theta Q_{1}-\left(A_{11}(\zeta, \varepsilon)-\lambda\right) P_{1}-A_{12}(\zeta, \varepsilon) P_{2} \\
\dot{P}_{2} & =Q_{2} \\
\dot{Q}_{2} & =-\varepsilon \theta Q_{2}-A_{21}(\zeta, \varepsilon) P_{1}-\left(A_{22}(\zeta, \varepsilon)-\lambda\right) P_{2} .
\end{aligned}
$$

Let $Y=\left(P_{1}, Q_{1}, P_{2}, Q_{2}\right)^{t}$ and let $A(\zeta, \lambda, \varepsilon)$ denote the coefficient matrix obtained by multiplying the first two equations by $\varepsilon^{-1}$. Then $(3.3)_{s}$ can be expressed as

$$
\dot{Y}=A(\zeta, \lambda, \varepsilon) Y
$$

Since (3.2) is a system we must allow $\lambda$ to be complex so that $Y$ lies in $\mathbb{C}^{4}$.

The associated fast linearized system is obtained by defining

$$
\begin{gathered}
p_{i}(\xi)=P_{i}(\varepsilon \xi), \quad q_{i}(\xi)=Q_{i}(\varepsilon \xi), \quad i=1,2, \\
a_{i j}(\xi, \varepsilon)=A_{i j}(\varepsilon \xi, \varepsilon) .
\end{gathered}
$$

The equations satisfied by the fast variables are easily seen to be

$$
\begin{aligned}
& p_{1}^{\prime}=q_{1}, \\
& q_{1}^{\prime}=-\theta q_{1}-\left(a_{11}(\xi, \varepsilon)-\lambda\right) p_{1}-a_{12}(\xi, \varepsilon) p_{2}, \\
& p_{2}^{\prime}=\varepsilon q_{2} \\
& q_{2}^{\prime}=\varepsilon\left[-\theta \varepsilon q_{2}-a_{21}(\xi, \varepsilon) p_{1}-\left(a_{22}(\xi, \varepsilon)-\lambda\right) p_{2}\right],
\end{aligned}
$$

or

$$
y^{\prime}=a(\xi, \lambda, \varepsilon) y,
$$

where $y=\left(p_{1}, q_{1}, p_{2}, q_{2}\right)^{t}$ and $a(\xi, \lambda, \varepsilon)$ is the fast coefficient matrix in $(3.3)_{f}$.

B. Asymptotic systems. For $\varepsilon>0, x(\xi, \varepsilon)$ tends to the origin and to the rest point at $C$, as $\xi$ tends to $-\infty$ and to $+\infty$, respectively. The matrices $a(\xi, \lambda, \varepsilon)$ and $A(\xi, \lambda, \varepsilon)$ therefore tend to limits $a^{ \pm}(\lambda, \varepsilon)$ and $A^{ \pm}(\lambda, \varepsilon)$ as $\xi \rightarrow \pm \infty$, where

$$
a^{ \pm}(\lambda, \varepsilon)=\left(\begin{array}{cccc}
0 & 1 & 0 & 1 \\
\left(-a_{11}^{ \pm}+\lambda\right) & -\theta & -a_{12}^{ \pm} & 0 \\
0 & 0 & 0 & \varepsilon \\
-\varepsilon a_{21}^{ \pm} & 0 & \varepsilon\left(-a_{22}^{ \pm}+\lambda\right) & -\varepsilon^{2} \theta
\end{array}\right)
$$




$$
A^{ \pm}(\lambda, \varepsilon)=\varepsilon^{-1} a^{ \pm}(\lambda, \varepsilon)
$$

here, $a_{i j}^{ \pm}=a_{i j}^{ \pm}(\lambda, \varepsilon)$ are the limits of $a_{i j}(\xi, \lambda, \varepsilon)$ at $\xi= \pm \infty$. The structure of $a^{ \pm}(\lambda, \varepsilon)$ is crucial in the analysis of the linearized equations. For example, these matrices determine the essential spectrum of $L(\varepsilon)$.

Note that the entries $a_{11}^{ \pm}$and $a_{22}^{ \pm}$are both negative while $a_{12}^{+}<0$ and $a_{21}^{+}>0$; also $a_{12}^{-}=0$. This information is easily seen from Figure 2.1. Define $\beta<0$ and a half-plane $\Omega \subset \mathbb{C}$ by

$$
\beta=\max \left\{a_{11}^{-}, a_{22}^{-}, a_{11}^{+}, a_{22}^{+}\right\}, \quad \Omega=\{\lambda \in \mathbb{C}: \operatorname{Re} \lambda>\beta\} .
$$

Lemma 3.1. For $\lambda \in \Omega$ and $\varepsilon>0$ sufficiently small, $a^{ \pm}(\lambda, \varepsilon)$ and $A^{ \pm}(\lambda, \varepsilon)$ each have four distinct eigenvalues $\mu_{i}^{ \pm}(\lambda, \varepsilon), 1 \leq i \leq 4$, and $\Delta_{i}(\lambda, \varepsilon), 1 \leq i \leq$ 4, respectively, which satisfy

$$
\begin{aligned}
& \operatorname{Re} \mu_{4}^{ \pm}<\operatorname{Re} \mu_{3}^{ \pm}<0<\operatorname{Re} \mu_{2}^{ \pm}<\operatorname{Re} \mu_{1}^{ \pm}, \\
& \Delta_{i}(\lambda, \varepsilon)=\varepsilon^{-1} \mu_{i}(\lambda, \varepsilon), \quad 1 \leq i \leq 4 .
\end{aligned}
$$

For small $\varepsilon, \mu_{2}^{ \pm}$and $\mu_{3}^{ \pm}$are $O(\varepsilon)$ while $\mu_{1}^{ \pm}, \mu_{4}^{ \pm}$remain $O(1)$. Let $e_{i}^{ \pm}(\lambda, \varepsilon)$ be an eigenvector of $a^{ \pm}(\lambda, \varepsilon)$ associated with $\mu_{i}^{ \pm}(\lambda, \varepsilon)$. Then $e_{i}^{ \pm}(\lambda, \varepsilon)$ tends to limits as $\varepsilon \rightarrow 0$, denoted by $e_{i R}^{ \pm}(\lambda)$, which are given by the expressions

$$
\left\{\begin{array}{l}
e_{1 R}^{ \pm}(\lambda)=\left(1, M_{p}^{ \pm}, 0,0\right)^{t}, \quad e_{4 R}^{ \pm}(\lambda)=\left(1, M_{n}^{ \pm}, 0,0\right)^{t}, \\
e_{2 R}^{ \pm}(\lambda)=\left(\frac{a_{12}^{ \pm}}{\lambda-a_{11}^{ \pm}}, 0,1, m_{p}^{ \pm}\right)^{t}, \quad e_{3 R}^{ \pm}(\lambda)=\left(\frac{a_{12}^{ \pm}}{\lambda-a_{11}^{ \pm}}, 0,1, m_{n}^{ \pm}\right)^{t},
\end{array}\right.
$$

where $a_{i j}^{ \pm}$are the entries of the Jacobian of $\left(u_{1} f_{1}, u_{2} f_{2}\right)$ at $U=0, C$ and

$$
\left\{\begin{array}{l}
M_{n}^{ \pm}, M_{p}^{ \pm}=-\theta^{*} / 2 \pm \frac{1}{2}\left(\theta^{* 2}+4\left(\lambda-a_{11}^{ \pm}\right)\right)^{1 / 2} \\
m_{n}^{ \pm}, m_{p}^{ \pm}= \pm\left[\lambda-a_{22}^{ \pm}-a_{12}^{ \pm} a_{21}^{ \pm} /\left(\lambda-a_{11}^{ \pm}\right)\right]^{1 / 2}
\end{array}\right.
$$

The subscript $n$ (resp. $p$ ) denotes the root with negative (resp. positive) real part.

Proof. Let $e=\left(c_{1}, c_{2}, c_{3}, c_{4}\right)^{t}$ be an eigenvector of $a^{ \pm}(\lambda, \varepsilon)$ associated with an eigenvalue $\mu=\varepsilon m$, where $m$ remains bounded as $\varepsilon \rightarrow 0$. It follows that $c_{2}=\varepsilon m c_{1}$ and that $c_{3}=m c_{4}$. For convenience we shall drop the $(\lambda, \varepsilon)$ dependence. The equation $a^{ \pm} e=\varepsilon m e$ leads to the equation

$$
p(m, \varepsilon) \stackrel{\text { def }}{=}\left(m^{2}+\varepsilon \theta m+\left(a_{22}^{ \pm}-\lambda\right)\right)\left(\varepsilon^{2} m^{2}+\varepsilon m \theta+a_{11}^{ \pm}-\lambda\right)-a_{12}^{ \pm} a_{21}^{ \pm}=0 .
$$

Clearly, $p(m, 0)$ has two distinct roots $m_{n}^{ \pm}(\lambda)$ and $m_{p}^{ \pm}(\lambda)$ with expressions as in (3.7c). Since $a_{i i}^{ \pm}<0$ and $a_{12}^{ \pm} a_{21}^{ \pm} \leq 0$ it follows from (3.6) that for $\lambda \in \Omega$ the quantities under the radicals in $(3.7 \mathrm{c})$ have positive real part, so that $m_{n}^{ \pm}(\lambda)$ has negative real part and $m_{p}^{ \pm}(\lambda)$ has positive real part. Furthermore, for $\varepsilon>0$ these roots perturb smoothly to distinct roots of $p(m, \varepsilon)$, yielding an expansion for the slow eigenvalues

$$
\mu_{2}^{ \pm}(\lambda, \varepsilon)=\varepsilon m_{p}^{ \pm}(\lambda)+\mathscr{O}\left(\varepsilon^{2}\right), \quad \mu_{2}^{ \pm}(\lambda, \varepsilon)=\varepsilon m_{p}^{ \pm}(\lambda)+\mathscr{O}\left(\varepsilon^{2}\right)
$$

which imply the middle inequalities in $(3.7 \mathrm{a})$. 
A similar argument provides an estimate for the fast eigenvalues. In particular, if $\mu=\mu(\lambda, \varepsilon)$ is an eigenvalue which remains $\mathscr{O}(1)$ as $\varepsilon \rightarrow 0$ then the equation for $\mu$ is

$$
\left(\mu^{2}+\theta \varepsilon^{2} \mu+\varepsilon^{2}\left(a_{22}^{ \pm}-\lambda\right)\right)\left(\mu^{2}+\theta \mu+a_{11}^{ \pm}-\lambda\right)-\varepsilon^{2} a_{12}^{ \pm} a_{21}^{ \pm}=0 .
$$

At $\varepsilon=0$ the roots are given explicitly by $(3.7 \mathrm{c})$, and for $\varepsilon>0$ we obtain the expansion

$$
\mu_{1}^{ \pm}(\lambda, \varepsilon)=M_{p}^{ \pm}(\lambda)+\mathscr{O}(\varepsilon), \quad \mu_{4}^{ \pm}(\lambda, \varepsilon)=M_{n}^{ \pm}(\lambda)+\mathscr{O}(\varepsilon) .
$$

The explicit formulae for the limits $e_{i R}^{ \pm}(\lambda)$ of the eigenfunctions follow from the above and some simple algebra, which we omit.

Remark 3.2. Suppose that $x$ is any point in the slow manifolds $S_{L}$ or $S_{R}$, let $u$ denote the $u$-components of $x$, and let $a(u, \lambda, \varepsilon)$ be the matrix in (3.4) with $a_{i j}=a_{i j}(u)$ equal to the entries of the Jacobian of $\left(u_{1} f_{1}, u_{2} f_{2}\right)$ at $u$. It is easily checked that $a_{11}(u), a_{12}(u)<0$ and $a_{21}(u)>0$, so that the eigenvalues $\mu_{1}(u, \lambda, \varepsilon)$ and $\mu_{4}(u, \lambda, \varepsilon)$ of $a(u, \lambda, \varepsilon)$ are distinct and satisfy inequalities (3.7a). If $a_{22}(u)<0$ then a similar remark applies to $\mu_{2}(u, \lambda, \varepsilon)$ and $\mu_{3}(u, \lambda, \varepsilon)$. However, it can occur that $a_{22}(u)>0$ in $S_{R}$ near $u_{2}=\alpha$, so that $\mu_{2}$ and $\mu_{3}$, together with their associated eigenvectors can coalesce. Hence the fast-slow structure is maintained whenever $x(\xi, \varepsilon)$ lies near $S_{L}$ or $S_{R}$, but hyperbolicity within the slow subspace can break down. However there will always exist a two-dimensional generalized slow subspace, which depends smoothly on the various parameters.

C. The spectrum of $L(\varepsilon)$. The spectrum $\sigma(\varepsilon)$ of $L(\varepsilon)$ consists of two sets: the essential spectrum $\sigma_{e}(\varepsilon)$ and the point spectrum $\sigma_{p}(\varepsilon)$. For differential operators of this type it is well known that $\sigma_{e}(\varepsilon)$ is contained between curves defined by the conditions that some eigenvalue $\mu_{i}^{ \pm}(\lambda, \varepsilon)$ is pure imaginary (see Henry [H]). By Lemma 3.1 these curves lie in the complement of $\Omega$ and, hence, $\sigma_{e}(\varepsilon)$ lies in the stable half-plane $\operatorname{Re} \lambda \leq \beta<0$ for small $\varepsilon$. The remainder of the spectrum, $\sigma_{p}(\varepsilon)$, consists of isolated eigenvalues of finite multiplicity.

Definition. $\lambda \in \Omega$ is an eigenvalue of $L(\varepsilon)$ if there exists a nontrivial, uniformly bounded solution $y(\xi)$ of $(3.3)_{f}$.

In view of the hyperbolicity of the asymptotic systems at $\xi= \pm \infty$, it is easily seen that $\lambda \in \Omega$ is an eigenvalue if and only if the bounded solution $y(\xi)$ decays to zero at $\xi= \pm \infty$ (see $[\mathrm{AGJ}])$.

D. The stable and unstable bundles. Armed with Lemma 3.1 we apply a result of [AGJ] asserting the existence of certain subspaces of solutions of $(3.3)_{f}$ which consist of solutions that decay as $\xi \rightarrow-\infty$ or as $\xi \rightarrow+\infty$. In particular, Lemmas 3.3, 3.5 in [AGJ] together with Lemma 3.1 above provides two twodimensional subspaces of solutions of $(3.3)_{f}$, which we denote by $\varphi^{-}(\xi, \lambda, \varepsilon)$ 


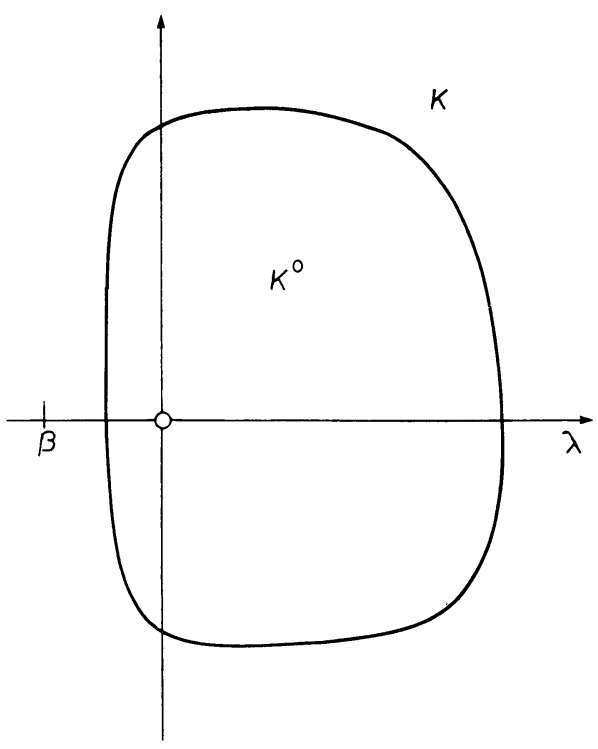

FIGURE 3.1

and by $\varphi^{+}(\xi, \lambda, \varepsilon)$, whose defining conditions are

$$
\begin{aligned}
& \varphi^{-}(\xi, \lambda, \varepsilon) \rightarrow \operatorname{span}\left\{e_{1}^{-}(\lambda, \varepsilon), e_{2}^{-}(\lambda, \varepsilon)\right\} \quad \text { as } \xi \rightarrow-\infty, \\
& \varphi^{+}(\xi, \lambda, \varepsilon) \rightarrow \operatorname{span}\left\{e_{3}^{+}(\lambda, \varepsilon), e_{4}^{+}(\lambda, \varepsilon)\right\} \quad \text { as } \xi \rightarrow+\infty
\end{aligned}
$$

the convergence is in the topology on the Grassmannian $G_{2,4}$. Thus $\varphi^{-}$is asymptotic to the unstable subspace of $a^{-}(\lambda, \varepsilon)$ at $\xi=-\infty$, while $\varphi^{+}$is asymptotic to the stable subspace of $a^{+}(\lambda, \varepsilon)$ and consists of solutions which decay as $\xi \rightarrow+\infty$. These subspaces can each be viewed as (complex) 2-plane bundles over the base space $(\xi, \lambda) \in \mathbb{R}^{1} \times \Omega$. We see that $\lambda \in \Omega$ is an eigenvalue if and only if $\varphi^{-}$and $\varphi^{+}$intersect nontrivially at $\lambda$.

Definition. $\varphi^{-}(\xi, \lambda, \varepsilon)$ is the unstable bundle associated with $(3.3)_{f}$ and $\varphi^{+}(\xi, \lambda, \varepsilon)$ is the stable bundle associated with $(3.3)_{f}$.

E. Outline of the proof of the Stability Theorem. Let $K \subset \mathbb{C}$ be a simple closed curve containing the origin in its interior $K^{0}$. The following lemma, whose proof follows immediately from Proposition 2.2 in [AGJ], shows that an eigenvalue count of $L(\varepsilon)$ inside $K$ is sufficient to determine the linearized stability of the wave.

Lemma 3.3. (a) Suppose that $K$ contains a region of the form

$$
\{\lambda \in \mathbb{C}:|\lambda| \leq R \text { and }|\arg \lambda| \leq \pi / 2+\delta\}
$$

for all sufficiently small $\delta>0$. There exists $\delta, R>0$ which are independent of $\varepsilon$ for $0<\varepsilon \leq 1$, such that $L(\varepsilon)$ has no eigenvalues $\lambda$ in the region exterior to $K$ in the sector $|\arg \lambda| \leq \pi / 2+\delta$. 
(b) If $L(\varepsilon)$ has exactly one simple eigenvalue at $\lambda=0$ inside $K$ (due to translation invariance), then the wave is (nonlinearly) stable, in the sense of the Stability Theorem.

The remainder of the paper is devoted to obtaining an eigenvalue count of $L(\varepsilon)$ inside the fixed curve $K$ of the lemma. The principal construction which will be employed in obtaining this information is that of a certain 2-plane bundle $\mathscr{E}(\varepsilon)$, called the augmented unstable bundle, obtained from $\varphi^{-}$whose base space is a real 2-sphere, which we describe briefly below. This construction is done rigorously in [AGJ].

First, the infinite variable $\xi$ is compactified through a change of variables $\xi \rightarrow \tau$, where $\tau(\xi)$ is the solution of

$$
\tau^{\prime}=\varepsilon \kappa\left(1-\tau^{2}\right), \quad \tau(0)=0
$$

where $\kappa>0$ and $\tau( \pm \infty)= \pm 1$. The bundle $\varphi^{-}$can now be regarded as a 2-plane over $(-1,1) \times \Omega$. We restrict $\lambda$ to lie along $K$ and consider the restriction $\varphi^{-} \mid(-1,1) \times K$ of $\varphi^{-}$.

The next step is to show that $\varphi^{-} \mid(-1,1) \times K$ can be continuously extended to a bundle on $[-1,1] \times K$. Before describing this construction, we pause to describe what is meant by a continuous extension. There is a very natural association between certain complex $k$-plane bundles over a base space $B$ and a map from $B$ into the Grassmannian $G_{k, n}$, which is the space of $k$-dimensional subspaces of $\mathbb{C}^{n}$ (see [MS]). This map works for subbundles of the trivial bundle $B \times \mathbb{C}^{n}$. Given a complex $k$-plane bundle

$$
\mathscr{E}: E \stackrel{\pi}{\longrightarrow} B,
$$

where $E \subset B \times \mathbb{C}^{n}$, we can associate with $\mathscr{E}$ a map $\hat{e}$ from $B$ into the Grassmannian $G_{k, n}$ of complex $k$-planes in $\mathbb{C}^{n}$, which assigns to each $b \in B$ the $k$-dimensional subspace of $\mathbb{C}^{n}$ which is the fiber above the point $b$. There is a natural bundle $\Gamma_{k}\left(\mathbb{C}^{n}\right)$ over $G_{k, n}$, called the universal bundle: given $g \in$ $G_{k, n}$ the fiber in $\Gamma_{k}\left(\mathbb{C}^{n}\right)$ is just the $k$-plane associated with $g$. A standard construction in differential geometry is the pullback of a bundle, we note then that the bundle $\mathscr{E}$ can be realized as the pullback $\hat{e}^{*} \Gamma_{k}\left(\mathbb{C}^{n}\right)($ see $[\mathrm{A}])$ :

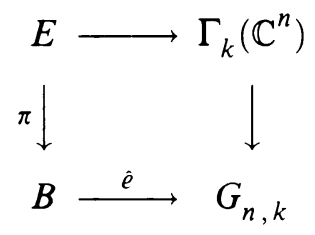

We say that $\varphi^{-} \mid(-1,1) \times K$ continuously extends to a bundle over $[-1,1] \times K$ if the associated map from $(-1,1) \times K$ into $G_{2,4}$ extends continuously to $[-1,1] \times K$.

Let

$$
U^{ \pm}(\lambda, \varepsilon)=\operatorname{span}\left\{e_{1}^{ \pm}(\lambda, \varepsilon), e_{2}^{ \pm}(\lambda, \varepsilon)\right\}
$$


denote the unstable subspaces at $\tau=-1$ and at $\tau=+1$. The defining condition for $\varphi^{-}$as $\tau \rightarrow-1$ ensures that $\varphi^{-}$continuously extends to $U^{-}(\lambda, \varepsilon)$ as $\tau \rightarrow-1$ for all $\lambda \in \Omega$; in particular, this is true for all $\lambda \in K$. However, at $\tau=+1$ this is not necessarily true; we need to make the further assumption that $\lambda$ is not an eigenvalue of $(3.3)_{f}$ for all $\lambda \in K$. Under this assumption it is shown in [AGJ, Lemma 3.7] that $U^{+}(\lambda, \varepsilon)$ extends to $\varphi^{-}$continuously as $\tau \rightarrow+1$.

We now have a bundle $\varphi^{-}$over a finite cylinder $[1,1] \times K$. The last step is to glue on "caps" over the ends $\tau= \pm 1$. The fibers over points in the caps are $U^{ \pm}(\lambda, \varepsilon)$ for $(\tau, \lambda) \in\{ \pm 1\} \times K^{0} . \mathscr{E}(\varepsilon)$ is the resulting bundle; its base space

$$
B=\{-1\} \times K^{0} \cup[-1,1] \times K \cup\{+1\} \times K^{0}
$$

is homeomorphic to $S^{2}$.

The principal result in [AGJ] equates the number of eigenvalues inside $K$ (counting algebraic multiplicity) with the first Chern number $c_{1}(\mathscr{E}(\varepsilon))$, of $\mathscr{E}(\varepsilon)$. The relevant topology is developed in [AGJ]. However, for the purpose of this paper, it will be sufficient to accept as given the existence of a homotopy invariant $c_{1}$ associated with the bundle $\mathscr{E}(\varepsilon)$ which counts the number of eigenvalues inside $K$, together with two basic properties of $c_{1}$ described in (a) and (b) below. More precisely, the principal goals of the remainder of the paper are the following:

(a) The decomposition of $\mathscr{E}(\varepsilon)$ into a Whitney sum

$$
\mathscr{E}(\varepsilon)=\mathscr{E}_{1}(\varepsilon) \oplus \mathscr{E}_{2}(\varepsilon)
$$

of complex line bundles $\mathscr{E}_{1}(\varepsilon)$ and $\mathscr{E}_{2}(\varepsilon)$. The fast-slow structure of the differential equations provides a natural means of obtaining such a Whitney sum decomposition. The information needed to obtain the decomposition is described in a little more detail in subsection $\mathrm{F}$, below. Given such a decomposition, $c_{1}(\mathscr{E}(\varepsilon))$ is the sum of $c_{1}\left(\mathscr{E}_{i}(\varepsilon)\right), i=1,2$.

(b) The computation of the classes $c_{1}\left(\mathscr{E}_{i}(\varepsilon)\right), i=1,2$. This step exploits the homotopy invariance of $c_{1}$ by continuing the computation to certain reduced bundles $\mathscr{E}_{1 R}$ and $\mathscr{E}_{2 R}$, which are defined directly from the equations with $\varepsilon=0$. The equations associated with these bundles are two-dimensional rather than four-dimensional and their eigenvalue problems can be studied by standard techniques, such as Sturm-Liouville theory. Hence to compute $c_{1}$ of the reduced bundles it suffices to obtain an eigenvalue count of the reduced equations and to apply the Chern number theorem in [AGJ] in reverse.

F. Complex line bundles and $\mathbb{C} P^{3}$. It will be convenient to describe the complex line bundles $\mathscr{E}_{i}(\varepsilon), i=1,2$, mentioned in the previous subsection in terms of maps $\hat{e}_{i}$ of a base space $B$ into $G_{1,4}$, which is complex projective space, $\mathbb{C} P^{3}$. The space $\mathbb{C} P^{3}$ is $\mathbb{C}^{4} \backslash\{0\}$ with the equivalence relation $y_{1} \sim y_{2}$ if $y_{1}, y_{2} \in \mathbb{C}^{4} \backslash\{0\}$ and $y_{1}=\alpha y_{2}$ for some $\alpha \in \mathbb{C}$; i.e., $\mathbb{C} P^{3}$ is the space of complex lines in $\mathbb{C}^{4}$. Let $\pi: \mathbb{C}^{4} \backslash\{0\} \rightarrow \mathbb{C} P^{3}$ be the projection map; for a 
given set $S \subset \mathbb{C}^{4} \backslash\{0\}$ we denote by $\widehat{S}$ its image $\pi(S)$ in $\mathbb{C} P^{3}$. A complex line bundle $\mathscr{E}_{i}$ over a space $B$ is the pullback $\hat{e}_{i}^{*} \Gamma_{1}\left(\mathbb{C}^{4}\right)$ of a map $\hat{e}_{i}$ into $\mathbb{C} P^{3}$ :

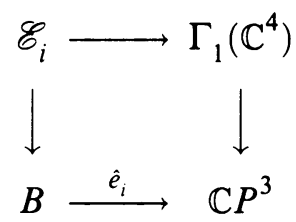

Given a linear system $y^{\prime}=b(\xi) y$ on $\mathbb{C}^{4}$, where $b(\xi)$ is a $4 \times 4$ matrix, there is an associated induced flow on $\mathbb{C} P^{3}$, which we denote by

$$
\hat{y}^{\prime}=\hat{b}(\hat{y}, \xi) \text {. }
$$

The fast and slow unstable bundles $\mathscr{E}_{1}(\varepsilon)$ and $\mathscr{E}_{2}(\varepsilon)$ are subbundles of $\mathscr{E}(\varepsilon)$ which will be defined later via certain solutions $\hat{e}_{1}$ and $\hat{e}_{2}$ of the projectivized system associated with $(3.3)_{f}$. With a slight abuse of terminology, we shall refer to the maps $\hat{e}_{1}$ and $\hat{e}_{2}$ themselves as the fast unstable bundle and the slow unstable bundle, respectively.

The fast and slow unstable bundles $\mathscr{E}_{1}(\varepsilon)$ and $\mathscr{E}_{2}(\varepsilon)$ are defined via $\hat{e}_{1}$ and $\hat{e}_{2}$ on the sides, $(-1,1) \times K$. The defining conditions for $\hat{e}_{1}$ and $\hat{e}_{2}$ are both at $\tau=-1$ and so they can automatically be capped off at $\{-1\} \times K^{0}$ by extending these maps to equal $\hat{e}_{1}^{-}(\lambda, \varepsilon)$ and $\hat{e}_{2}^{-}(\lambda, \varepsilon)$ on this set. The difficulty is in extending $\hat{e}_{1}$ and $\hat{e}_{2}$ to $\tau= \pm 1$; this requires a description of the global behavior of $\hat{e}_{1}$ and $\hat{e}_{2}$ as solutions of the projectivized equations associated with $(3.3)_{f}$. In particular, the information required in obtaining the Whitney sum decomposition of $\mathscr{E}(\varepsilon)$ is

$$
\lim _{\tau \rightarrow+1} \hat{e}_{i}(\tau, \lambda, \varepsilon)=\hat{e}_{i}^{+}(\lambda, \varepsilon) \quad(\lambda \in K, i=1,2) .
$$

This is proved in $\S \S \mathrm{IV}$ and $\mathrm{V}$.

G. The reduced equations. The fast and slow reduced bundles will be defined from the equations obtained by setting $\varepsilon=0$ in $(3.3)_{f}$ and (3.3) . It is important to first set the parametrizations for the underlying waves: $x(\xi, \varepsilon)$ and $x_{R}(\xi)$ in the fast scaling, and $X(\zeta, \varepsilon)$ and $X_{R}(\zeta)$ in the slow scaling. Recall that $x$ and $x_{R}$ are both determined by setting $u_{1}(0, \varepsilon)=u_{1 R}(0)=h$ for some fixed $h \in(0, p(\alpha))$. This also determines $X(\zeta, \varepsilon)=x(\zeta / \varepsilon, \varepsilon)$; finally, set $X_{R}(\zeta)$ so that the discontinuity occurs at $\zeta=0$.

The fast reduced system is

$$
\begin{aligned}
& p_{1 R}^{\prime}=q_{1 R}, \\
& q_{1 R}^{\prime}=-\theta^{*} q_{1 R}-\left(a_{11}^{R}(\xi)-\lambda\right) p_{1 R}-a_{12}^{R}(\xi) p_{2 R}, \\
& p_{2 R}^{\prime}=0, \\
& q_{2 R}^{\prime}=0 ;
\end{aligned}
$$

the coefficients $a_{i j}^{R}(\xi)$ are the entries of the Jacobian matrix of $\left(u_{1} f_{1}, u_{2} f_{2}\right)$ evaluated at $x_{R}(\xi)$. In view of the parametrization for $x(\xi, \varepsilon)$ and $x_{R}(\xi, \varepsilon)$ 
it follows from Corollary 2.2 that the coefficients of $(3.3)_{f}$ tend to those of (3.9) $f R$ uniformly for compact $\xi$.

The slow reduced system is obtained by setting $\varepsilon=0$ in $(3.3)_{s}$, to obtain the algebraic-differential system

$$
\begin{aligned}
0 & =Q_{1}, \\
0 & =\left(\lambda-A_{11}^{R}(\zeta)\right) P_{1}-A_{12}^{R}(\zeta) P_{2}, \\
\dot{P}_{2} & =Q_{2}, \\
\dot{Q}_{2} & =\left[\frac{A_{12}^{R}(\zeta) A_{21}^{R}(\zeta)}{\lambda-A_{11}^{R}(\zeta)}+A_{22}^{R}(\zeta)-\lambda\right] P_{2} ;
\end{aligned}
$$

here, $A_{i, j}^{R}(\zeta)$ are the entries of the Jacobian matrix of $\left(u_{1} f_{1}, u_{2} f_{2}\right)$ evaluated at $X_{R}(\zeta)$. Recall from $\S I I C$ that the $U_{1}$-component of $X_{R}(\zeta)$ is discontinuous across $\zeta=0$. It follows that solutions $\left(P_{2}, Q_{2}\right)$ of the differential equations in (3.9) $)_{s R}$ will be continuous across $\zeta=0$, but that $P_{1}$ will in general be discontinuous. We remark that by Corollary 2.2 and our parametrization of $X(\zeta, \varepsilon), X_{R}(\zeta)$, it follows that the coefficients of (3.3) send uniformly to those of (3.9) $)_{s R}$ for $\zeta$ bounded away from zero.

\section{The estimation of $\hat{e}_{1}$ AND the ELEPHANT TRUNK LeMma}

A. Preliminaries. Consider the projectivized equations associated with $(3.3)_{f}$, which we will denote by

$$
\hat{y}^{\prime}=\hat{a}(\hat{y}, \xi, \lambda, \varepsilon) .
$$

The fast unstable bundle $\hat{e}_{1}$ will be defined to be a certain solution of (4.1). In order to define $\hat{e}_{1}$ it will be useful to recall some facts concerning autonomous linear systems on $\mathbb{C}^{n}$ and their projectivizations on $\mathbb{C} P^{n-1}$ :

$$
\begin{gathered}
y^{\prime}=b y \quad\left(y \in \mathbb{C}^{n}\right), \\
\hat{y}^{\prime}=\hat{b}(\hat{y}) \quad\left(\hat{y} \in \mathbb{C} P^{n-1}\right) .
\end{gathered}
$$

Clearly, $e$ is an eigenvector of the $n \times n$ complex matrix $b$ if and only if $\hat{e}$ is a critical point of the vector field $\hat{b}(\hat{y})$. It is easily checked that if $\mu_{i}$ are the eigenvalues of $b$ with associated eigenvectors $e_{i}$, then $d \hat{b}\left(\hat{e}_{i}\right)$ is a linear operator on $\mathbb{C}^{n-1}$ with eigenvalues $\mu_{j}-\mu_{i}, j \neq i$ (see e.g. [J]). In particular, if $\mu_{1}$ is the eigenvalue of largest real part, then $\hat{e}_{1}$ is an attracting critical point, as can easily be seen in Figure 4.1 depicting a linear saddle point at the origin.

The asymptotic system for (4.1) at $\xi=-\infty$ has an attracting critical point $\hat{e}_{1}^{-}(\lambda, \varepsilon)$ for all $\lambda \in \Omega$, since $\mu_{1}^{-}(\lambda, \varepsilon)$ is the eigenvalue of $a^{-}(\lambda, \varepsilon)$ of largest real part. It follows from general arguments presented in [AGJ, §3.B] that there is a unique solution $\hat{e}_{1}(\xi, \lambda, \varepsilon)$ of the nonautonomous equations (4.1) which satisfies

$$
\lim _{\xi \rightarrow-\infty} \hat{e}_{1}(\xi, \lambda, \varepsilon)=\hat{e}_{1}^{-}(\lambda, \varepsilon) .
$$

This condition uniquely defines the unstable bundle $\hat{e}_{1}$. 


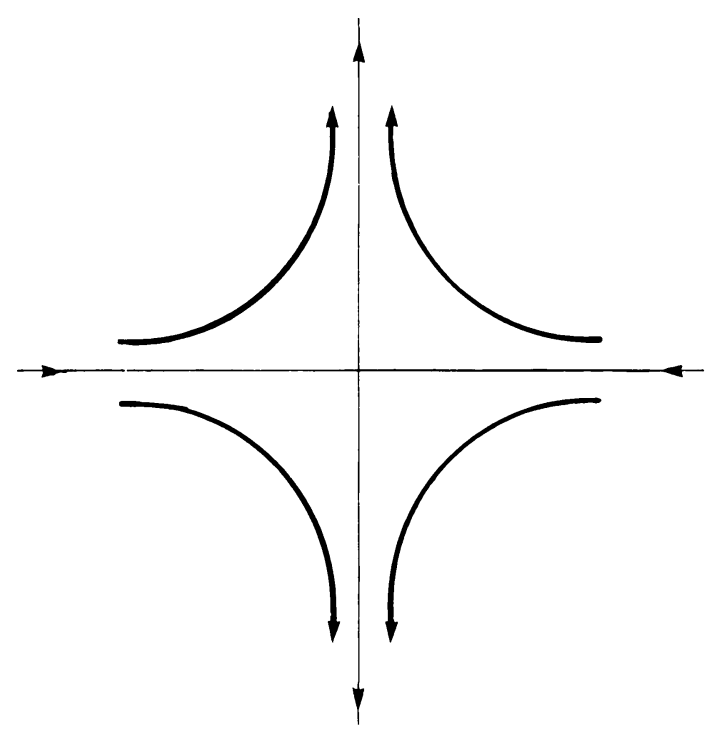

FIGURE 4.1

The remainder of this section is devoted to characterizing the global behavior of $\hat{e}_{1}$ and, in particular, its behavior as $\xi \rightarrow+\infty$. The idea is to construct a positively invariant set $\widehat{\Omega}^{u} \subset \mathbb{C} P^{3} \times \mathbb{R}$ for the equations (4.1), augmented with $\xi^{\prime}=1$ which, by virtue of the defining condition for $\hat{e}_{1}$ at $\xi=-\infty$, contains $\hat{e}_{1}$ for all $\xi$. This set is constructed as a union of tubes

$$
\widehat{\Omega}^{u}=\widehat{\Omega}_{L} \cup \widehat{\Omega}_{F} \cup \widehat{\Omega}_{R}
$$

(see Figure 4.2); the slow tubes $\widehat{\Omega}_{L}, \widehat{\Omega}_{R}$ enable us to track $\hat{e}_{1}$ over the leftand right-hand slow manifolds, while $\widehat{\Omega}_{F}$ contains $\hat{e}_{1}$ in the transition layer. The slow tubes are neighborhoods in $\mathbb{C} P^{3}$ of the fast unstable eigenvector of the coefficient matrix $a(\xi, \lambda, \varepsilon)$.

Near the slow manifolds we will show that $\hat{e}_{1}$ remains in a region in $\mathbb{C} P^{3}$ where $p_{1} \neq 0$ in the fibers $\pi^{-1} \hat{e}_{1}$. It is therefore convenient to introduce the local coordinate system

$$
\tilde{y}=(1, \beta), \quad \beta=\left(\frac{q_{1}}{p_{1}}, \frac{p_{2}}{p_{1}}, \frac{q_{2}}{p_{1}}\right)=\left(\beta_{1}, \beta_{2}, \beta_{3}\right),
$$

where $y \in \pi^{-1} \hat{y}$. The equations for $\beta$ assume the form

$$
\begin{aligned}
& \beta_{1}^{\prime}=-\theta \beta_{1}-\left(a_{11}(\xi, \varepsilon)-\lambda\right)-a_{12}(\xi, \varepsilon) \beta_{2}-\beta_{1}^{2}, \\
& \beta_{2}^{\prime}=\varepsilon \beta_{3}-\beta_{1} \beta_{2}, \\
& \beta_{3}^{\prime}=\varepsilon\left[-\varepsilon \theta \beta_{3}-a_{21}(\xi, \varepsilon)-\left(a_{22}(\xi, \varepsilon)-\lambda\right) \beta_{2}-\beta_{1} \beta_{3}\right] .
\end{aligned}
$$

These coordinates will frequently be used whenever the underlying wave is near the slow manifolds. With a slight abuse of notation, given $\hat{y} \in \mathbb{C} P^{3}$ such that $p_{1} \neq 0$ in the fiber over $\hat{y} \in \mathbb{C} P^{3}$ denote by $\tilde{y}$ the element of $\pi^{-1} \hat{y}$ whose $p_{1}$-component equals one. 
B. Relatively invariant sets for nonautonomous systems. We shall prove a general lemma that will be needed in the construction of $\widehat{\Omega}_{L}$ and $\widehat{\Omega}_{R}$ concerning nonautonomous systems of the form

$$
\beta^{\prime}=F(\beta, \xi, \varepsilon) \quad\left(\beta \in \mathbb{C}^{n}\right),
$$

where $F$ depends weakly on $\xi$ if $\varepsilon$ is sufficiently small (see (4.7)). Suppose that $\Omega \subset \mathbb{C}^{n} \times \mathbb{R}$ is a set of the form

$$
\Omega=\bigcup_{\xi \in I} \Omega(\xi) \times\{\xi\},
$$

where $I$ is an open interval in $\mathbb{R}^{1}$ and $\Omega(\xi)$ is a family of neighborhoods in $\mathbb{C}^{n}$, such that $\partial \Omega \cap \mathbb{C}^{n} \times I$ is a smooth mánifold (i.e., $\partial \Omega(\xi)$ varies smoothly with $\xi)$.

Definition. $\Omega$ is positively invariant relative to $I$ if for any solution $\beta(\xi)$ of (4.4) with $\beta\left(\xi_{0}\right) \in \Omega\left(\xi_{0}\right)$ for some $\xi_{0} \in I$, then $\beta(\xi) \in \Omega(\xi)$ for all $\xi \geq \xi_{0}$ for which $\xi \in I$.

In order to construct relatively invariant sets for (4.4) we shall consider the family of frozen systems,

$$
\frac{d \beta}{d \xi}=F(\beta, \gamma ; \varepsilon) \quad(\gamma \in I)
$$

Suppose that for each $\gamma \in I,(4.5)_{\gamma}$ admits a critical point $\beta_{0}(\gamma, \varepsilon)$ such that $\beta_{0}$ depends smoothly on $\gamma$ and that there exists $\alpha>0$ independent of $\varepsilon$ and $\xi \in I$ such that

$$
\operatorname{Re} \sigma\left[d_{\beta} F\left(\beta_{0}(\gamma, \varepsilon), \gamma, \varepsilon\right)\right]<-\alpha .
$$

Here $\sigma$ denotes the spectrum of the Jacobian $d_{\beta} F$. We shall also assume that given any $d>0$ there exists $\varepsilon_{0}=\varepsilon_{0}(d)$ and a nested family of subintervals $I(d) \subset I$ such that $I\left(d_{1}\right) \subset I\left(d_{2}\right)$ for $d_{1}<d_{2}$ and

$$
\sup _{(\beta, \xi, \varepsilon) \in C}\left\{\left|F_{\xi}\right|,\left\|F_{\beta, \xi}\right\|,\left|\beta_{0 \xi}\right|\right\}<d,
$$

where the set $C$ is given by

$$
C=\left\{(\beta, \xi, \varepsilon):\left|\beta-\beta_{0}(\xi, \varepsilon)\right|<c_{0}, \xi \in I(d), 0<\varepsilon \leq \varepsilon_{0}\right\} .
$$

The constant $c_{0}$ need only be chosen so that $\beta$ is in the domain of $F$; in our application we may take $c_{0}=1$. We also assume that

$$
K=\sup _{C}\left\|d^{2} F(\beta, \xi, \varepsilon)\right\|<\infty \text {. }
$$

Next, let

$$
A(\gamma)=d_{\beta} F\left(\beta_{0}(\gamma, \varepsilon), \gamma, \varepsilon\right)
$$

(we shall suppress the $\varepsilon$-dependence from now on). We shall assume that there exists an invertible matrix $Y(\gamma)$ which depends smoothly on $\gamma$ such that the 
following is true. Let $\langle\cdot, \cdot\rangle_{\gamma}$ and $|\cdot|_{\gamma}$ be the inner product and norm on $\mathbb{C}^{n}$ defined by

$$
\left\langle\beta_{1}, \beta_{2}\right\rangle_{\gamma}=Y(\gamma) \beta_{1} \cdot \overline{Y(\gamma) \beta_{2}}, \quad|\beta|_{\gamma}=\langle\beta, \beta\rangle_{\gamma}^{1 / 2} .
$$

We shall assume that there exists $a>0$ depending only on $\alpha$ in (4.6) such that

$$
\left\{\begin{array}{l}
\operatorname{Re}\langle A(\gamma) \beta, \beta\rangle_{\gamma}<-a|\beta|_{\gamma}^{2}, \\
\|Y(\gamma)\|_{\gamma}=1, \quad\left\|Y_{\gamma}(\gamma)\right\|_{\gamma} \leq d .
\end{array}\right.
$$

In the event that $A(\gamma)$ has a basis of eigenvectors which depends smoothly on $\gamma$ we may take $Y(\gamma)$ to be the inverse of this matrix. However, in our applications, Remark 3.2 shows that the eigenvalues $\mu_{2}-\mu_{1}$ and $\mu_{3}-\mu_{1}$ of this matrix can coalesce over certain portions of $S_{R}$, and the (generalized) eigenvectors of $A(\gamma)$ may depend discontinuously on $\gamma$. We will verify that a suitable $Y(\gamma)$ can be found in the next section. Finally, given $\eta>0$ and $d>0$, define $\Omega \subset \mathbb{C}^{n} \times \mathbb{R}$ by

$$
\Omega=\Omega(d, \eta)=\left\{(\beta, \gamma):\left|\beta-\beta_{0}(\gamma, \varepsilon)\right|_{\gamma}<\eta, \gamma \in I(d)\right\} .
$$

It will be useful later on to keep in mind that $d$ is associated with the slowly varying character of (4.4) while $\eta$ is associated with the width of the tube $\Omega$.

Lemma 4.1. With notation and hypotheses as above, and, in particular, (4.6), (4.7), (4.8), and (4.9), there exists $\eta_{0}$ depending only on $K$ in (4.8), and there exists $B>0$ depending only on $\eta_{0}, K$, and $\alpha$ in (4.6), such that if $\eta \leq \eta_{0}$ and if $0<d<B \eta$ then $\Omega(d, \eta)$ in (4.10) is positively invariant relative to $I(d)$ for the equations (4.4).

Proof. Let $\beta(\xi)$ be a solution of (4.4) with $\beta\left(\xi_{*}\right)=\beta_{*}$, where $\left(\beta_{*}, \xi_{*}\right) \in$ $\Omega(d, \eta)$. Suppose that $d<B \eta$ and that the lemma is false. Set $g(\xi)=$ $\left|\beta(\xi)-\beta_{0}(\xi, \varepsilon)\right|_{\xi}$; then $g\left(\xi_{*}\right)<\eta$ and there exists a smallest $t \in I(d)$ with $t>\xi_{*}$ such that $g(t)=\eta$. We will show that $g^{\prime}(t)<0$, contradicting the minimality of $t$. From the definition of $|\cdot|_{\xi}$, it follows that

$$
g^{\prime}(t)=\eta^{-1}\left[Y\left(\beta-\beta_{0}\right) \cdot \overline{Y_{\xi}\left(\beta-\beta_{0}\right)}-Y\left(\beta-\beta_{0}\right) \cdot \overline{Y \beta_{0 \xi}}+Y\left(\beta-\beta_{0}\right) \cdot \overline{Y f(\beta, \xi, \varepsilon)}\right]_{\xi=t} .
$$

Since $g^{\prime}(t)$ is real valued, we may replace the right-hand side of (4.11) with its real part. Now

$$
F(\beta, t, \varepsilon)=A(t)\left(\beta-\beta_{0}\right)+R\left(\beta-\beta_{0}\right),
$$

where $\left|R\left(\beta-\beta_{0}\right)\right|_{t} \leq C\left|\beta-\beta_{0}\right|_{t}^{2}$ and $C$ depends on $d_{\beta}^{2} F$ and is therefore dominated by $K$ in (4.8). In the following we shall let $C>0$ denote a generic constant which depends on any of the various quantities in the statement of the lemma. By (4.9) we have that $\left\|Y_{\xi}\right\|_{\xi}<d$, where $\|\cdot\|_{\xi}$ is the matrix norm induced by $|\cdot|_{\xi}$. It now follows from (4.11) and (4.9) that

$$
g^{\prime}(t) \leq C \eta d+C d+(C \eta-a) \eta
$$


for some constant $C$. Choose $\eta_{0}=a /(2 C)$ and $B=a / 2 C\left(\eta_{0}+1\right)$; it then follows that $g^{\prime}(t)<0$ provided that $\eta<\eta_{0}$ and $d<B \eta$, yielding the desired contradiction.

C. Relatively invariant sets for (4.3). If $\xi$ and $\varepsilon$ are such that $x(\xi, \varepsilon)$ lies near either slow manifold $S_{L}$ or $S_{R}$ then by Remark 3.2 it follows that the eigenvalues $\mu_{i}(\xi, \lambda, \varepsilon)$ of $a(\xi, \lambda, \varepsilon)$ satisfy the estimate (3.7a). Let $f_{1}(\xi, \lambda, \varepsilon)$ be an eigenvector of $a(\xi, \lambda, \varepsilon)$ associated with the eigenvalue $\mu_{1}(\xi, \lambda, \varepsilon)$ of largest real part, normalized so that $\left|f_{1}(\xi, \lambda, \varepsilon)\right|_{\infty}=1$ for each $(\xi, \lambda, \varepsilon)$. We pick the usual parametrization for $x(\xi, \varepsilon)$, namely $u_{1}(0, \varepsilon)=h$. It follows that there exists $\bar{\xi}>0$ depending only on $h$ such that $f_{1}(\xi, \lambda, \varepsilon)$ will be well defined whenever $|\xi| \geq \bar{\xi}$. From the estimate in (3.7b) it follows for $|\xi| \geq \bar{\xi}$ and $\varepsilon$ sufficiently small, that the $p_{1}$-component of $f_{1}$ remains $\mathscr{O}(1)$ as $\varepsilon \rightarrow 0$. Without loss of generality, it may be assumed that the $p_{1}$-component of $f_{1}$ equals 1 so that $f_{1}=\tilde{f}_{1}$. We may therefore use the local coordinates $\beta$ in (4.2) to describe the solution $\hat{y}(\xi)$ of $(4.1)$ whenever $\tilde{y}(\xi)$ is near $f_{1}(\xi, \lambda, \varepsilon)$ for all small $\varepsilon>0$. The relevant equations are therefore (4.3), which we may express more succinctly as

$$
\beta^{\prime}=F(\beta, \xi, \lambda, \varepsilon) .
$$

For $|\gamma| \geq \bar{\xi}$ and all $\varepsilon>0$ sufficiently small there exists a curve $\beta_{0}(\gamma, \lambda, \varepsilon)$ of critical points of the frozen systems associated with (4.12),

$$
\beta^{\prime}=F(\beta, \xi, \lambda, \varepsilon),
$$

consisting of the $\left(q_{1}, p_{2}, q_{2}\right)$ components of $f_{1}(\xi, \lambda, \varepsilon)$. By a previous remark, if

$$
A(\xi, \lambda, \varepsilon)=d_{\beta} F\left(\beta_{0}(\xi, \lambda, \varepsilon), \xi, \lambda, \varepsilon\right),
$$

then for $|\xi| \geq \bar{\xi}$ and small $\varepsilon>0, A$ has eigenvalues $\mu_{i}-\mu_{1}, i=1,2,3,4$, at $(\xi, \lambda, \varepsilon)$ and if $\bar{\xi}$ is large enough, estimate (4.6) will hold uniformly for all $|\xi|>\bar{\xi}$ and for small $\varepsilon$ for some $\alpha>0$. Since the branch $\beta_{0}(\xi, \lambda, \varepsilon)$ is uniformly bounded for all $|\xi|>\bar{\xi}, \lambda \in K$, and all sufficiently small $\varepsilon$, it follows that (4.8) holds for some $K$, with $c_{0}$ in the definition of $C$ equal to 1. Also, we take the interval $I$ to be either

$$
I_{L}=\{\xi:-\infty<\xi<-\bar{\xi}\} \text { or } I_{R}=\{\xi: \bar{\xi}<\xi<\infty\} .
$$

Next, we verify (4.7) for a suitable family of subintervals $I_{L}(d) \subset I_{L}$ and $I_{R}(d) \subset I_{R}$. Note the $F(\beta, \xi, \lambda, \varepsilon)$ depends on $\xi$ through the dependence of the $a_{i j}(\xi, \varepsilon)$ on the $u$-components of $x(\xi, \varepsilon)$. Hence there exists a constant $L>0$ such that

$$
\max \left\{\left|F_{\xi}\right|,\left\|F_{\xi, \beta}\right\|,\left|\beta_{0 \xi}\right|\right\}<L \max \left\{\left|u_{1}^{\prime}(\xi, \varepsilon)\right|, u_{2}^{\prime}(\xi, \varepsilon)\right\}
$$

for all sufficiently small $\varepsilon>0$, say $0<\varepsilon \leq \varepsilon_{0}$. Now let

$$
n(\delta)=n_{-}(\delta) \cup n_{F}(\delta) \cup n_{+}(\delta) \times I_{\delta}
$$


be the tube constructed about the singular limit in $§ I I E$. By resetting $\varepsilon_{0}$ if necessary and choosing $\delta>0$ small enough, there exist $\xi_{L}(\delta) \leq-\bar{\xi}$ and $\xi_{R}(\delta) \geq \bar{\xi}$ such that for $0<\varepsilon \leq \varepsilon_{0}$,

$$
\begin{array}{ll}
x(\xi, \varepsilon) \in n_{-}(\delta) & \text { for } \xi \leq \xi_{L}(\delta) \text { and } x\left(\xi_{L}(\delta), \varepsilon\right) \in n_{-}(\delta) \cap n_{F}(\delta), \\
x(\xi, \varepsilon) \in n_{+}(\delta) & \text { for } \xi \geq \xi_{R}(\delta) \text { and } x\left(\xi_{R}(\delta), \varepsilon\right) \in n_{+}(\delta) \cap n_{F}(\delta) .
\end{array}
$$

We claim that there exists a constant $M>0$ such that for $\xi \leq \xi_{L}(\delta)$ or $\xi \geq \xi_{R}(\delta)$ and $\varepsilon \leq \varepsilon_{0}$,

$$
\left|u_{1}^{\prime}(\xi, \varepsilon)\right|,\left|u_{2}^{\prime}(\xi, \varepsilon)\right| \leq M \delta .
$$

Since $u_{2}^{\prime}=\varepsilon v_{2}$ and $v_{2}$ is uniformly bounded along the entire wave, (4.15) will hold for $u_{2}^{\prime}$ by setting $M_{2} \geq\left(\varepsilon_{0} / \delta\right) \max \left|v_{2}\right|$. Similarly, since $u_{1}^{\prime}=v_{1}$, it trivially follows for $x \in n_{ \pm}(\delta)$ that $\left|v_{1}\right| \leq \delta$, so that we may take $M=$ $\max \left\{1, M_{2}\right\}$.

Finally, given any small $d>0$, set $\delta(d)=(M L)^{-1} d$ and

$$
I_{L}(d)=\left\{\xi: \xi \leq \xi_{L}(\delta(d))\right\}, \quad I_{R}(d)=\left\{\xi: \xi_{R}(\delta(d)) \leq \xi\right\} .
$$

The set $C$ in (4.7) is defined with $I(d)$ equal to either $I_{L}(d)$ or $I_{R}(d)$. Combining (4.1) and (4.15) yields (4.7), as required. In the following we shall write

$$
\xi_{L}(d)=\xi_{L}(\delta(d)), \quad \xi_{R}(d)=\Omega_{R}(\delta(d)) ;
$$

these should be thought of as the times $\xi$ when $x(\xi, \varepsilon)$ is (approximately) a distance $d$ away from the left and right corners of the singular limit.

Next, set $\eta_{0}$ and $B$ as in Lemma 4.1. For $0<\eta<\eta_{0}$ and $0<d<B \eta$ define

$$
\begin{array}{ll}
\Omega_{L}=\Omega_{L}(d, \eta ; \lambda, \varepsilon) & \text { as in (4.10) with } I(d)=I_{L}(d), \\
\Omega_{R}=\Omega_{R}(d, \eta ; \lambda, \varepsilon) & \text { as in (4.10) with } I(d)=I_{R}(d) .
\end{array}
$$

To complete the construction we need to produce a family of matrices $Y=$ $Y(\gamma, \lambda, \varepsilon)$ so that the associated norms and inner products on $\mathbb{C}^{3}$ satisfy the inequalities in (4.9). To this end we compute the linearization of $F$ at $\tilde{f}_{1}$. It follows from $(3.7 b)$ that

$$
A(\xi, \lambda, \varepsilon)=\left(\begin{array}{ccc}
-\Delta(\xi, \lambda, \varepsilon) & -a_{12}(\xi, \varepsilon) & 0 \\
0 & -M_{p}(\xi, \lambda, \varepsilon) & 0 \\
0 & 0 & -M_{p}(\xi, \lambda, \varepsilon)
\end{array}\right)+B(\xi, \lambda, \varepsilon),
$$

where $B(\xi, \lambda, \varepsilon)$ is a $3 \times 3$ matrix whose entries are uniformly of order $\delta$,

$$
\begin{gathered}
M_{p}(\xi, \lambda, \varepsilon)=-\theta / 2+\frac{1}{2} \Delta(\xi, \lambda, \varepsilon), \\
\Delta(\xi, \lambda, \varepsilon)=\left[\theta^{2}+4\left(\lambda-a_{11}(\xi, \varepsilon)\right)\right]^{1 / 2} .
\end{gathered}
$$

Since $a_{11}$ is strictly negative along the slow manifolds $S_{L}, S_{R}$, it follows that $\Omega$ can be modified if necessary so that $\operatorname{Re}\left(\lambda-a_{11}\right)$ is positive for $\lambda \in \Omega$. Similar considerations also imply that $\Delta \neq M_{p}$ for $\lambda \in \Omega$ and all $(\xi, \varepsilon)$ such 
that the underlying wave is near the slow manifolds. It follows that both $\Delta$ and $M_{p}$ have positive real part so that $A$ is negative definite, and also that $A$ is diagonalizable for such $(\xi, \lambda, \varepsilon)$. We can therefore take

$$
Y(\xi, \lambda, \varepsilon)=\left(\begin{array}{ccc}
1 & a_{12}(\xi, \varepsilon) & 0 \\
0 & -\Delta(\xi, \lambda, \varepsilon)+M_{p}(\xi, \lambda, \varepsilon) & 0 \\
0 & 0 & 1
\end{array}\right)
$$

it then follows that $Y^{-1} A Y$ is a matrix with diagonal entries of uniformly negative real part and with all remaining entries of order $\delta$. The inequalities in (4.9) follow from this.

We summarize this result below.

Lemma 4.2. Let $\xi_{L}(d), \xi_{R}(d), \Omega_{L}$, and $\Omega_{R}$ be defined as above with $d<B \eta$. Then there exists $\varepsilon_{0}>0$ such that $\Omega_{L}$ and $\Omega_{R}$ are positively invariant relative to $I_{L}(d)$ and to $I_{R}(d)$, respectively.

With a slight abuse of notation, we shall write $\widehat{\Omega}_{L}$ and $\widehat{\Omega}_{R}$ for the images of $\Omega_{L}$ and $\Omega_{R}$ in $\mathbb{C} P^{3} \times \mathbb{R}$.

D. The elephant trunk lemma. We are now equipped to prove the main result of this section. Our goal is to construct positively and negatively invariant sets $\widehat{\Omega}^{u}$ and $\widehat{\Omega}^{s}$, respectively, in $\mathbb{C} P^{3} \times \mathbb{R}$, for the projectivized system (4.1) augmented with $\xi^{\prime}=1$. $\widehat{\Omega}^{u}$ will be used to locate the solution $\hat{e}_{1}(\xi, \lambda, \varepsilon)$ and to characterize its behavior as $\xi \rightarrow+\infty$; $\widehat{\Omega}^{s}$ will play a similar role for $\hat{e}_{4}$ as $\xi \rightarrow-\infty$. Recall that $\hat{e}_{1}$ and $\hat{e}_{4}$ are the fast unstable and the fast stable bundles associated with $(3.3)_{f}$, respectively. We shall supply the description of and the argument for $\widehat{\Omega}^{u}$; the argument for the repelling set $\widehat{\Omega}^{s}$ is the same as for $\widehat{\Omega}^{u}$ after a time reversal and will be omitted.

The principal construction that needs to be described is that of a set $\widehat{\Omega}_{F}$ that will describe $\hat{e}_{1}$ over the transition layer; in addition, $\hat{\Omega}_{F}$ will connect the two sets $\widehat{\Omega}_{L}$ and $\widehat{\Omega}_{R}$ defined in the previous section. It is here that the choice of $\lambda$ is crucial. We shall assume that $\lambda$ lies on a curve $K \subset \Omega$ enclosing the origin as in Figure 3.1. The criterion for choosing $K$ is that it should not contain eigenvalues of the fast reduced system $(3.9)_{f R}$. Since this system is degenerate we need to make this more precise.

Definition. $\lambda \in \mathbb{C}$ is an eigenvalue of $(3.9)_{f R}$ if $p_{2 R} \equiv q_{2 R} \equiv 0$ and if the system

$$
p_{1 R}^{\prime}=q_{1 R}, \quad q_{1 R}^{\prime}=-\theta^{*} q_{1 R}-\left(a_{11}^{R}(\xi)-\lambda\right) p_{1 R}
$$

has a nontrivial uniformly bounded solution.

Note that (4.17) is equivalent to the second order equation

$$
p_{1 R}^{\prime \prime}+\theta_{1 R}^{\prime}+\frac{\partial}{\partial u_{1}}\left(u_{1 R}(\xi) f_{1}\left(u_{1 R}(\xi), \alpha\right) p_{1 R}=\lambda p_{1 R} .\right.
$$


These equations are the linearization about the travelling wave solution $\left(u_{1 R}(\xi)\right.$, $\left.v_{1 R}(\xi)\right)$ of a scalar, bistable reaction-diffusion equation consisting of the 1components of $(2.3)_{f R}$. It is well known that such waves are stable. In fact, if $b=\max a_{11}^{R}( \pm \infty)<0$, then the spectrum of (4.17) in the half-plane $\operatorname{Re} \lambda \geq b$ consists of a simple eigenvalue at $\lambda=0$. These results are proved in the paper [FM] by Fife and McLeod. Thus a suitable choice $K \subset \Omega$ of a curve enclosing $\lambda=0$ can be found on which there are no eigenvalues of $(3.9)_{f R}$.

The region $\widehat{\Omega}^{u}$ can now be defined. Let $\xi_{L}(d)$ and $\xi_{R}(d)$ be the functions defined in $\S$ IVC, let $\eta_{L}, \eta_{R}<\eta_{0}$ and $d_{L}<B \eta_{L}, d_{R}<B \eta_{R}$, where $\eta_{0}$ and $B$ are as in Lemma 4.1; finally set

$$
\widehat{\Omega}_{L}=\widehat{\Omega}_{L}\left(d_{L}, \eta_{L} ; \lambda, \varepsilon\right), \quad \widehat{\Omega}_{R}=\widehat{\Omega}_{R}\left(d_{R} ; \eta_{R} ; \lambda, \varepsilon\right)
$$

as in (4.16). Of particular importance is the "bottom" of $\widehat{\Omega}_{R}$, which we define to be

$$
\hat{\omega}_{R}=\hat{\omega}_{R}\left(d_{R}, \eta_{R}, \lambda, \varepsilon\right)=\widehat{\Omega}_{R} \cap \mathbb{C} P^{3} \times\left\{\xi_{R}\left(d_{R}\right)\right\} .
$$

Let $\hat{\omega}_{F} \subset \hat{\omega}_{R}$ be a neighborhood in $\hat{\omega}_{R} ; \hat{\omega}_{F}$ will be specified below (see (4.23)). The set $\hat{\Omega}_{F} \subset \mathbb{C} P^{3} \times \mathbb{R}$ is defined to be

$$
\begin{aligned}
\widehat{\Omega}_{F}=\{(\hat{y}(\xi), \xi): & \hat{y}(\xi) \text { satisfies }(4.1)_{f}, \\
& \left.\left(\hat{y}\left(\xi_{R}\right), \xi_{R}\right) \in \hat{\omega}_{F}, \text { and } \xi_{L} \leq \xi \leq \xi_{R}\right\},
\end{aligned}
$$

where $\xi_{L}=\xi_{L}\left(d_{L}\right)$ and $\xi_{R}=\xi_{R}\left(d_{R}\right)$. Thus $\hat{\Omega}_{F}$ consists of solution segments of $(4.1)_{f}$ which lie in $\hat{\omega}_{F}$ at $\xi=\xi_{R}$. A dual construction can also be given for the transition region for $\widehat{\Omega}^{s}$. By construction the set $\widehat{\Omega}_{F}$ is a positively invariant relative to the interval $I=\left[\xi_{L}, \xi_{R}\right]$. The region $\widehat{\Omega}^{u}$ is defined to be

$$
\widehat{\Omega}^{u}=\widehat{\Omega}_{L} \cup \widehat{\Omega}_{R} \cup \widehat{\Omega}_{R} .
$$

The following theorem shows that $\widehat{\Omega}^{u}$ (resp. $\widehat{\Omega}^{s}$ ), and has the aspect depicted in Figure 4.2. In particular, $\widehat{\Omega}_{L} \cap\left\{\xi_{L}\right\}$ is interior to $\widehat{\Omega}_{F} \cap\left\{\xi_{L}\right\}$ so that the union $\widehat{\Omega}^{u}$ of the three relatively invariant sets in $(4.20)$ yields a positively invariant set. The aspect of the figure suggests a concatenation of elephants linked together trunk-to-tail.

Theorem 4.3 (the elephant trunk lemma). Suppose that $\lambda \in K$ is not an eigenvalue of (3.9) ${ }_{f R}$. There exist $d_{L}, d_{R}, \eta_{L}$, and $\eta_{R}$ satisfying $\eta_{L}, \eta_{R}<\eta_{0}$ and $d_{L}<B \eta_{L}, d_{R}<B \eta_{R}$, and there exists $\bar{\varepsilon}>0$ such that $\widehat{\Omega}^{u}$ is positively invariant and $\hat{\Omega}^{s}$ is negatively invariant for $(4.1)_{f}$ for $0<\varepsilon \leq \bar{\varepsilon}$ and all $\lambda \in K$.

Proof. We prove the theorem for $\widehat{\Omega}^{u}$. From the remark preceding the statement of the theorem it suffices to show that the set $\hat{\omega}_{F}$ in (4.18) can be chosen so that

$$
\widehat{\Omega}_{L} \cap \mathbb{C} P^{3} \times\left\{\xi_{L}\right\} \subset \widehat{\Omega}_{F} \cap \mathbb{C} P^{3} \times\left\{\xi_{L}\right\} .
$$



(a) $\widehat{\Omega}^{u}$
(b) $\widehat{\Omega}^{s}$

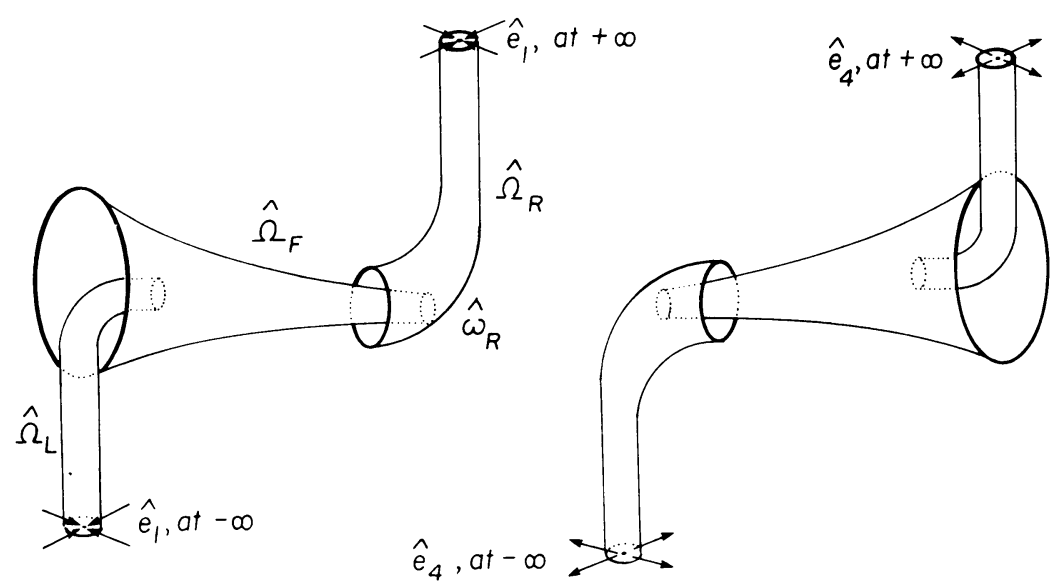

FIGURE 4.2

We will verify (4.21) for a suitable choice of the defining parameters $\eta_{L}, \eta_{R}$, $d_{L}$, and $d_{R}$. At various steps $\varepsilon$ will have to be set sufficiently small. This will be expressed by writing $\varepsilon \leq \bar{\varepsilon}$, where it is understood that $\bar{\varepsilon}$ is chosen smaller than in the previous step if necessary.

The set $\widehat{\Omega}_{F}$ is approximated by considering the projectivization of the reduced system $(3.9)_{f R}$, namely

$$
\hat{y}^{\prime}=\hat{a}(\hat{y}, \xi, \lambda, 0):=\hat{a}_{R}(\hat{y}, \xi, \lambda) .
$$

Recall that the condition $u_{1 R}(0)=h$ sets the parametrization for $(3.9)_{f R}$ and hence, for (4.22). Let $x_{R}(\xi)$ be the underlying wave in (2.4), and let $x_{R}^{ \pm}=x_{R}( \pm \infty)$ be the left- and right-hand corners of the singular limit. Let $a_{R}(\xi, \lambda)=a(\xi, \lambda, 0)$ be the coefficient matrix of $(3.9)_{f R}$ and let $e_{1 R C}^{ \pm}(\lambda)$ be the fast unstable eigenvectors of $a_{R}( \pm \infty, \lambda)$ in the left- and right-hand corners. (Note that $e_{1 R C}^{ \pm}(\lambda)$ is not the same as $e_{1 R}^{ \pm}(\lambda)$ in (3.7b).) Since $x_{R}^{ \pm}$lie in the slow manifolds, an analysis similar to the derivation of the characterization of $e_{1 R}^{ \pm}(\lambda)$ in (3.7b) yields an analogous characterization of $e_{1 R C}^{ \pm}(\lambda)$. By a general construction in [AGJ] there exists a unique solution $\hat{e}_{1 R}(\xi, \lambda)$ of $(4.22)$ such that $\hat{e}_{1 R}(\xi, \lambda)$ tends to $\hat{e}_{1 R C}^{-}(\lambda)$ as $\xi \rightarrow-\infty$. Since $\lambda \in K$ is not an eigenvalue of $(3.9)_{f R}$ it follows that the 1-components of $e_{1 R}(\xi, \lambda)$ become unbounded as $\xi \rightarrow+\infty$. This can occur if and only if $\hat{e}_{1 R}(\xi, \lambda)$ tends to $\hat{e}_{1 R C}^{+}(\lambda)$ as $\xi \rightarrow+\infty$. Thus $\hat{e}_{1 R}(\xi, \lambda)$ is an orbit of $(4.22)$ which connects $\hat{e}_{1 R C}^{-}(\lambda)$ to $\hat{e}_{1 R C}^{+}(\lambda)$.

First, set $0<\eta_{L}, \eta_{R}<\eta_{0}$ so that for $|\xi| \geq \bar{\xi}$, (4.6) will be satisfied for $0<\varepsilon \leq \bar{\varepsilon}$. Note that the size of each slice of $\widehat{\Omega}_{L}$ and $\widehat{\Omega}_{R}$ at fixed $\xi$ is uniform (either $\eta_{L}$ or $\eta_{R}$ ) for all $\xi \leq-\bar{\xi}$ and $\xi \geq \bar{\xi}$. The proof is based on the following three assertions. 
Claim 1. There exists $\bar{\varepsilon}>0$, a neighborhood $\hat{\omega}^{+}$of $\hat{e}_{1 R C}^{+}(\lambda)$ in $\mathbb{C} P^{3}$, and $d_{R}<B \eta_{R}$ such that

$$
\hat{\omega}_{F} \stackrel{\text { def }}{=} \hat{\omega}^{+} \times\left\{\xi_{R}\right\} \subset \hat{\omega}_{R}\left(d_{R}, \eta_{R} ; \lambda, \varepsilon\right)
$$

for $0<\varepsilon \leq \bar{\varepsilon}$.

Keeping $d_{R}, \eta_{R}$ fixed, let $T \leq \xi_{R}$; define

$$
\widehat{\omega}^{+}(\xi)=\left\{\hat{y}(\varepsilon): \hat{y}(\xi) \text { solves }(4.22) \text { and } \hat{y}\left(\xi_{R}\right) \in \hat{\omega}^{+}\right\} .
$$

Thus $\hat{\omega}^{+}(\xi)$ is $\omega^{+}$at time $\xi$ under the reduced flow.

Claim 2. Let $\hat{\omega}^{-}$be a small attracting neighborhood of $\hat{e}_{1 R C}^{-}(\lambda)$ for the asymptotic system associated with (4.22) at $\xi=-\infty$. There exists $T<-\bar{\xi}$ such that $\hat{\omega}^{-} \subset \hat{\omega}^{+}(\xi)$ for all $\xi \leq T$.

It follows from our parametrization of the underlying waves that $\xi_{L}\left(d_{L}\right) \rightarrow$ $-\infty$ as $d_{L} \rightarrow 0$; we can therefore select $d_{L}$ so small that $\xi_{L}\left(d_{L}\right)<T$, where $T$ is as in Claim 2. Finally, set

$$
\hat{\omega}_{L}=\hat{\omega}_{L}\left(d_{L}, \eta_{L} ; \lambda, \varepsilon\right)=\hat{\Omega}_{L}\left(d_{L}, \eta_{L} ; \lambda, \varepsilon\right) \cap \mathbb{C} P^{3} \times\left\{\xi_{L}\right\} .
$$

Claim 3. There exists $\bar{\varepsilon}>0, \eta_{L}<\eta_{0}$, and $d_{L}<B \eta_{L}$ such that $\hat{\omega}_{L} \subset \hat{\omega}^{-}$for $0<\varepsilon \leq \bar{\varepsilon}$.

Assuming the validity of the claims we can easily establish (4.21). With all defining parameters set as in the claims and $0<\varepsilon \leq \bar{\varepsilon}$ it suffices to show that

$$
\hat{\omega}^{-} \times\left\{\xi_{L}\right\} \subset \widehat{\Omega}_{F}\left(d_{R}, \eta_{R} ; \lambda, \varepsilon\right) \cap \mathbb{C} P^{3} \times\left\{\xi_{L}\right\} .
$$

Recall that $\widehat{\Omega}_{F}$ was defined by following solution curves $\hat{y}_{\varepsilon}(\xi)$ of $(4.1)_{f}$, with $\varepsilon>0$ and $\hat{y}_{\varepsilon}\left(\xi_{R}\right) \in \hat{\omega}^{+}$, backward in time to $\hat{y}_{\varepsilon}\left(\xi_{L}\right)$. Let $\hat{y}_{0}(\xi)$ be a solution of (4.22) with $\hat{y}_{0}\left(\xi_{R}\right)=\hat{y}_{\varepsilon}\left(\xi_{R}\right)$. It follows from Corollary 2.2 that the vector field $\hat{a}(\hat{y}, \xi, \lambda, \varepsilon)$ tends to $\hat{a}_{R}(\hat{y}, \xi, \lambda)$ as $\varepsilon \rightarrow 0$ uniformly on the interval $\xi_{L} \leq$ $\xi \leq \xi_{R}$, and from Gronwall's inequality that $\left|\hat{y}_{\varepsilon}(\xi)-\hat{y}_{0}(\xi)\right|$ tends uniformly to zero as $\varepsilon \rightarrow 0$ on the interval $\xi_{L} \leq \xi \leq \xi_{R}$. Hence the set on the right side of (4.25) approaches the set $\hat{\omega}^{+}\left(\xi_{L}\right)$ in Claim 2 as $\varepsilon \rightarrow 0$. Thus (4.25) follows from the above and Claim 2 for $0<\varepsilon \leq \bar{\varepsilon}$ for $\bar{\varepsilon}$ sufficiently small.

Proof of Claim 1. Let $f_{1 R}(\xi, \lambda)$ be the fast unstable eigenvector of $a(\xi, \lambda, 0)$; $f_{1 R}$ is well defined for $|\xi| \geq \xi$. By an estimate similar to (3.7b) we may scale $f_{1 R}$ so that $f_{1 R}=\hat{f}_{1 R}$. Since $x_{R}(\xi) \rightarrow x_{R}^{+}$it follows that $f_{1 R}(\xi, \lambda)$ tends to $e_{1 R C}^{+}(\lambda)$ as $\xi \rightarrow+\infty$. Now let $\hat{\omega}^{+}$be a fixed neighborhood of radius $\eta_{R} / 3$ about $\hat{e}_{1 R C}^{+}(\lambda)$ in $\mathbb{C} P^{3}$; here and below distance on $\mathbb{C} P^{3}$ is measured in the norm $|\cdot|_{\xi_{R}}$ defined earlier. From the above there exists $\xi_{0} \geq \bar{\xi}$ such that $\hat{f}_{1 R}(\xi, \lambda) \in \hat{\omega}^{+}$for all $\xi \geq \xi_{0}$.

Next consider $\hat{\omega}_{R}$; this is a disk of radius $\eta_{R}$ about $\hat{f}_{1}\left(\xi_{R}\left(d_{R}\right), \lambda, \varepsilon\right)$. Since $x\left(\xi_{R}, \varepsilon\right) \in n_{+}(\delta) \cap n_{F}(\delta)$ and $\delta=(M L)^{-1} d_{R}$ it follows that $\xi_{R} \rightarrow+\infty$ as $d_{R} \rightarrow 0$. Set $d_{R}>0$ so small that $\xi_{R}\left(d_{R}\right)>\xi_{0}$. By the parametrization set 


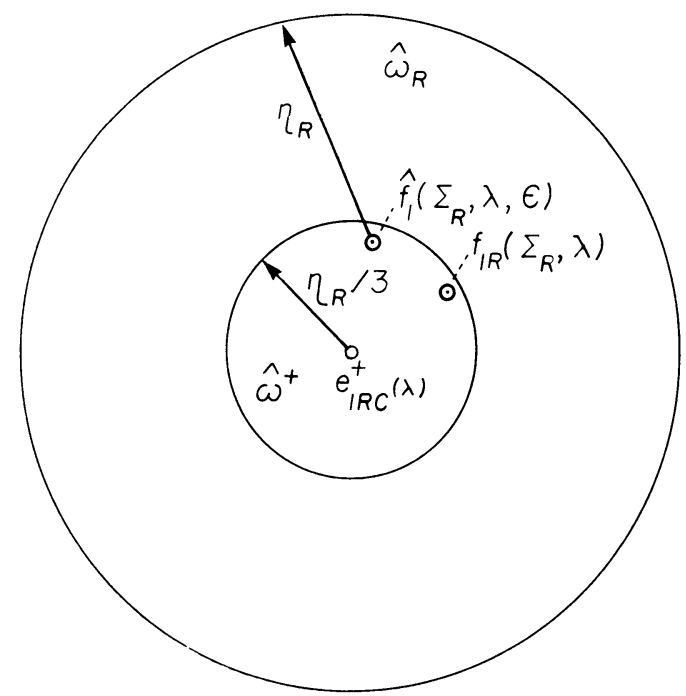

FIGURE 4.3

for the underlying waves $x(\xi, \varepsilon)$ it also follows that $\hat{f}_{1}(\xi, \lambda, \varepsilon) \rightarrow \hat{f}_{1 R}(\xi, \lambda)$ as $\varepsilon \rightarrow 0$, uniformly on compact $\xi$-intervals. Since $\xi_{R}\left(d_{R}\right) \geq \xi_{0}$ it follows from the first paragraph that $\hat{f}_{1 R}\left(\xi_{R}, \lambda\right) \in \hat{\omega}^{+}$. Finally, since $\hat{f}_{1 R}\left(\xi_{R}, \lambda, \varepsilon\right)$ tends to $\hat{f}_{1 R}\left(\xi_{R}, \lambda\right)$ as $\varepsilon \rightarrow 0$ there exists $\bar{\varepsilon}$ such that for $0<\varepsilon \leq \bar{\varepsilon}$ we have that $\hat{f}_{1}\left(\xi_{R}, \lambda, \varepsilon\right)$ lies within $\eta_{R} / 3$ of $\hat{f}_{1 R}\left(\xi_{R}, \lambda\right)$. Given any $\hat{p} \in \hat{\omega}^{+}$it then follows that

$$
\begin{aligned}
\left|\hat{p}-\hat{f}_{1}\left(\xi_{R}, \lambda, \varepsilon\right)\right|_{\xi_{R}}< & \left|\hat{p}-\hat{e}_{1 R C}(\lambda)\right|_{\xi_{R}}+\left|\hat{e}_{1 R C}(\lambda)-f_{1 R}\left(\xi_{R}, \lambda\right)\right|_{\xi_{R}} \\
& +\left|f_{1 R}\left(\xi_{R}, \lambda\right)-\hat{f}_{1}\left(\xi_{R}, \lambda, \varepsilon\right)\right|_{\xi_{R}} \\
< & \eta_{R} / 3+\eta_{R} / 3+\eta_{R} / 3=\eta_{R} .
\end{aligned}
$$

Proof of Claim 2. Let $\hat{e}_{1 R}(\xi, \lambda)$ be the solution of (4.22) which connects $e_{1 R C}^{-}(\lambda)$ to $e_{1 R C}^{+}(\lambda)$. It follows that

$$
\hat{e}_{1 R}(\xi, \lambda) \in \hat{\omega}^{+} \text {for all } \xi \geq \xi_{1}
$$

for some $\xi_{1} \geq \bar{\xi}$. Since $\xi_{R}\left(d_{R}\right) \rightarrow+\infty$ as $d_{R} \rightarrow 0$ we can assume that $d_{R}$ is reset if necessary so that $\xi_{R}\left(d_{R}\right)>\xi_{1}$. This may now require resetting $\bar{\varepsilon}$ in Claim 1; assume that this has been done.

We now compactify $\xi$ in (4.22) by introducing a change of variables $\tau=$ $\tau(\xi) ;(4.22)$ is written as

$$
\begin{aligned}
\hat{y}^{\prime} & =\hat{a}(\hat{y}, \tau, \lambda, 0), \\
\tau^{\prime} & =\varepsilon \kappa\left(1-\tau^{2}\right) \quad(-1<\tau<1) .
\end{aligned}
$$

Here, $\kappa>0$ is a small constant. From the second equation $\tau$ increases monotonically from $\tau=-1$ to $\tau=+1$; we set the reparametrization by requiring that $\tau(0)=0$. Then $\xi$ can be solved for in terms of $\tau$ and the vector field 


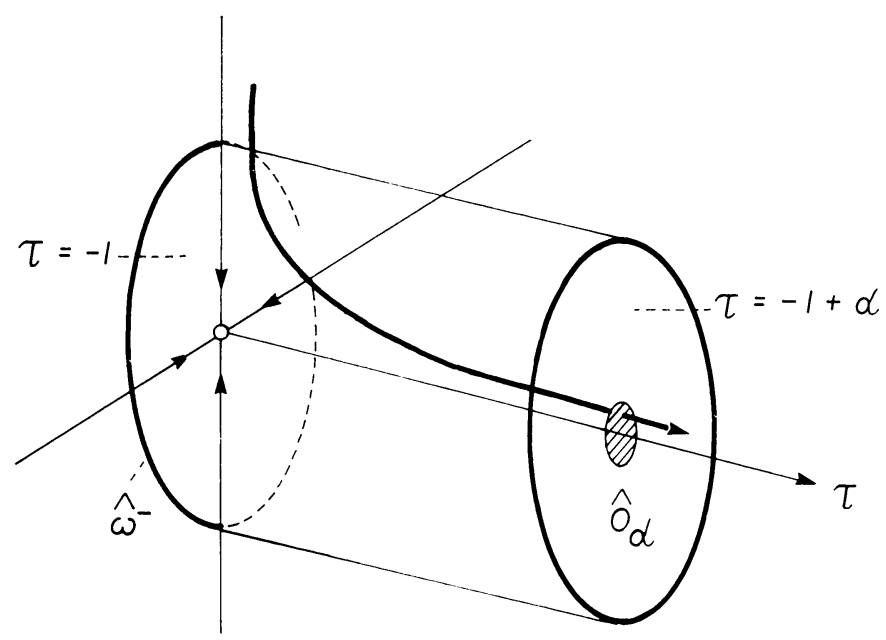

FIGURE 4.4

$\hat{a}(\hat{y}, \tau, \lambda, 0)$ is obtained from (4.15) by substituting the relation $\xi=\xi(\tau)$. The asymptotic systems of (4.22) correspond to the invariant manifolds $\mathbb{C} P^{3} \times\{ \pm 1\}$ of (4.26). Note that the rest point $\left(\hat{e}_{1 R C}^{-}(\lambda),-1\right)$ of $(4.26)$ is an attractor in the manifold $\mathbb{C} P^{3} \times\{-1\}$, while in the full space $\mathbb{C} P^{3} \times \mathbb{R}^{1}$ it is a saddle with a one(real) dimensional unstable manifold, which is tangent to the $\tau$-axis (see Figure 4.4). The solution $\left(\hat{e}_{1 R}(\tau, \lambda), \tau\right)$ of $(4.26)$ obtained from $e_{1 R}(\xi, \lambda)$ coincides with this unstable manifold for $-1<\tau<1$.

Let $\hat{\omega}^{-}$be a small attracting neighborhood of $\left(\hat{e}_{1 R C}^{-}(\lambda),-1\right)$ in $\mathbb{C} P^{3} \times\{-1\}$ (relative to the flow in this set). Let $C$ be the cylinder

$$
C=\hat{\omega}^{-} \times[-1,-1+\alpha] .
$$

If $\alpha>0$ is small enough, solutions of (4.26) enter $C$ through the sides $\partial \hat{\omega}^{-} \times$ $[-1,-1+\alpha]$ and leave $C$ through the face $\hat{\omega}^{-} \times\{-1+\alpha\}$. Finally, the face $\hat{\omega}^{-}\{-1\}$ is invariant and solutions in this face tend to the critical point in forward time.

Let $\xi_{\alpha}$ be the $\xi$ associated with $\tau=-1+\alpha$. By the first paragraph of the proof of this claim it follows that the set $\hat{\omega}^{+}\left(\xi_{\alpha}\right)$ defined in (4.24) with $\xi=\xi_{\alpha}$ is a neighborhood of $\hat{e}_{1 R}\left(\xi_{\alpha}, \lambda\right)$. Let $\mathscr{O}_{\alpha}=\hat{\omega}^{+}\left(\xi_{\alpha}\right) \times\{-1+\alpha\}$ be the associated neighborhood in the right face of $C$. Since $\left(\hat{e}_{1 R}(\tau, \lambda), \tau\right)$ is the only solution of (4.26) which remains in $C$ in backward time, and because at $\xi=\xi_{\alpha}$ it is interior to $\mathscr{O}_{\alpha}$ it follows that every solution of (4.26) through $\partial \mathscr{O}_{\alpha}$ leaves $C$ in finite time. Moreover, it follows from the compactness of $C$ and $\partial \mathscr{O}_{\alpha}$ that there exists $T \in\left(-\infty, \xi_{\alpha}\right)$ such that each such solution exits $C$ at a time $\xi \geq T$. Finally, the time map $\hat{\omega}^{+} \rightarrow \hat{\omega}^{+}(T)$ is a homeomorphism. Since $\hat{\omega}^{+}$ is a ball it follows that $\hat{\omega}^{+}(T)$ is also, and since $\partial \hat{\omega}^{+}(T)$ is exterior to the ball $\hat{\omega}^{-}$, it follows that $\hat{\omega}^{-} \subset \hat{\omega}^{+}(T)$. Without loss of generality we may choose $T \leq-\bar{\xi}$. 
Proof of Claim 3. We use the notation introduced in the proof of Claim 1. Since $\hat{e}_{1 R C}(\lambda) \in \hat{\omega}^{-}$and $\hat{f}_{1 R}(\xi, \lambda)$ tends to $\hat{e}_{1 R C}^{-}(\lambda)$ as $\xi \rightarrow-\infty$ there exists $\xi_{L} \leq T$ such that $\hat{f}_{1 R}(\xi, \lambda) \in \hat{\omega}^{-}$for all $\xi \leq \xi_{L}$. Select $\eta_{L}<\eta_{0}$ such that the disk about $\hat{f}_{1 R}\left(\xi_{L}, \lambda\right)$ of radius $\eta_{L}$ (in the $|\cdot|_{\xi_{L}}$ metric) is contained in $\hat{\omega}^{-}$. Since $\xi_{L}\left(d_{L}\right)$ tends to $-\infty$ as $d_{L} \rightarrow 0$ we can find $d_{L}<B \eta_{L}$ such that $\xi_{L}\left(d_{L}\right)<\xi_{L}$. Hence Claim 3 will be satisfied for such $d_{L}, \eta_{L}$ and for $0<\varepsilon \leq \bar{\varepsilon}$ sufficiently small.

Remark 4.4. The proof of a similar result was first obtained by Jones [J] for the FitzHugh-Nagumo equations. This is clearly a feature of fast-slow systems that is true in a general setting and it will undoubtedly be required in future applications of the theory in [AGJ]. It would be awkward to state a general theorem due to the many different ways in which the small parameter $\varepsilon$ can enter into the equations. Rather we shall try to identify the main features of the equations which were needed in the proof.

(a) The system supports the existence of a family of waves $x(\xi, \varepsilon)=X(\zeta, \varepsilon)$ for small $\varepsilon>0$ with two distinct time scales, each of which has clearly identifiable limits $x_{R}(\xi)$ and $X_{R}(\zeta)$ as $\varepsilon \rightarrow 0$. The slow limit $X_{R}(\zeta)$ is piecewise continuous and lies in the slow manifolds $S_{L}$ and $S_{R}$. The fast limit $x_{R}(\xi)$ describes transition layers in solutions jumping between $S_{L}$ and $S_{R}$.

(b) For some domain $\Omega \subset \mathbb{C}$ the coefficient matrix $a(\xi, \lambda, \varepsilon)$ of the linearized equations has a simple eigenvalue of largest real part for $\lambda \in \Omega$, whenever the underlying wave lies near either $S_{L}$ or $S_{R}$.

(c) The spectrum $\sigma_{R}$ for the linearized equations about the connecting orbit $x_{R}(\xi)$ of the reduced system is known, and $\lambda$ is chosen in $\Omega \backslash \sigma_{R}$.

The elephant trunk lemma yields the following characterization of the behavior of $\hat{e}_{1}$ for large $\xi$.

Corollary 4.5. Under the hypotheses of Theorem 4.3 it follows that

$$
\lim _{\xi \rightarrow+\infty} \hat{e}_{1}(\xi, \lambda, \varepsilon)=\hat{e}_{1}^{+}(\lambda, \varepsilon)
$$

for all $\lambda \in K$ and $0<\varepsilon \leq \bar{\varepsilon}$. The limit is uniform in $\lambda$ for $\lambda \in K$.

\section{THE DEFINITION AND APPROXIMATION OF $\hat{e}_{2}$}

A. Definition of $\hat{e}_{2}$. Generically, a solution $\hat{e}_{2}(\xi, \lambda, \varepsilon)$ of (4.1) which tends to $\hat{e}_{2}^{-}(\lambda, \varepsilon)$ as $\xi \rightarrow-\infty$ will tend to $\hat{e}_{1}^{+}(\lambda, \varepsilon)$ as $\xi \rightarrow+\infty$. We will show, however, that for a particular choice of the slow unstable bundle $\hat{e}_{2}$ we can arrange that $\hat{e}_{2}$ tends to $\hat{e}_{2}^{+}(\lambda, \varepsilon)$ as $\xi \rightarrow+\infty$. This will be crucial in obtaining the direct sum decomposition of the 2-plane bundle $\mathscr{E}(\varepsilon)$. The first step is to locate a choice of $\hat{e}_{2}$ whose asymptotic behavior at $\xi=+\infty$ is different from that of $\hat{e}_{1}$, i.e., its $\omega$ limit set lies in $\left\{\hat{e}_{i}(\lambda, \varepsilon), i=2,3,4\right\}$. 
Theorem 5.1. Suppose that $\lambda \in K$ is not an eigenvalue of (3.9) $)_{f R}$ and that $0<\varepsilon \leq \bar{\varepsilon}$. Then there exists a solution $\hat{e}_{2}(\xi, \lambda, \varepsilon)$ of (4.1) such that

(a) $\lim _{\xi \rightarrow-\infty} \hat{e}_{2}(\xi, \lambda, \varepsilon)=\hat{e}_{2}^{-}(\lambda, \varepsilon)$,

(b) $\lim _{\xi \rightarrow+\infty} \hat{e}_{2}(\xi, \lambda, \varepsilon) \in\left\{\hat{e}_{i}^{+}(\lambda, \varepsilon), i=2,3,4\right\}$.

These conditions uniquely determine $\hat{e}_{2}$.

Proof. We shall construct a 3-plane bundle of solutions $\psi^{+}$of solutions of $(3.3)_{f}$ over the cylinder $\mathbb{R}^{1} \times K$ such that every solution in $\psi^{+}$satisfies condition (b). This is done by compactifying the projectivized equations (4.1) by replacing $\xi$ with $\tau=\tau(\xi)$, yielding the system

$$
\hat{y}^{\prime}=\hat{a}(\hat{y}, \tau, \lambda, \varepsilon), \quad \tau^{\prime}=\varepsilon \kappa\left(1-\tau^{2}\right) \quad(-1 \leq \tau \leq 1) ;
$$

here $\kappa>0$ is chosen small enough that $\tau(\xi)$ decays to \pm 1 at $\xi= \pm \infty$ slower than the rate at which the underlying wave $x(\xi, \varepsilon)$ converges to its limits at $\pm \infty$. Under this condition, with $\tau=\tau(\xi)$ in $\hat{a}$, the vector field $\hat{a}$ is $C^{1}$ in its arguments (see [AGJ]). The critical points of $(5.1)$ are $\left(\hat{e}_{i}^{ \pm}(\lambda, \varepsilon), \pm 1\right)$ with $1 \leq$ $i \leq 4$. The bundle $\psi^{+}$is constructed by selecting certain solutions $e^{*}(\xi, \lambda, \varepsilon)$ in the stable manifolds of the rest points $\left(\hat{e}_{i}^{+}(\lambda, \varepsilon),+1\right)$ for $i=2,3,4$.

First consider $\left(\hat{e}_{4}^{+}(\lambda, \varepsilon),+1\right)$. Since $e_{4}^{+}(\lambda, \varepsilon)$ is the fastest stable eigenvector of $a^{+}(\lambda, \varepsilon)$ it follows that $e_{4}^{+}(\lambda, \varepsilon)$ is a repeller for the flow in the invariant slice $\tau \equiv+1$ of $(5.1)$. It follows that $\left(\hat{e}_{4}^{+}(\lambda, \varepsilon), 1\right)$ is a saddle with a (real) one-dimensional stable manifold. Let $\left(\hat{e}_{4}^{*}(\xi, \lambda, \varepsilon), \tau(\xi)\right)$ be the solution in this manifold with $\tau(\xi)<1$ and let $e_{4}^{*}(\xi, \lambda, \varepsilon)=\tilde{e}_{4}^{*}(\xi, \lambda, \varepsilon)$; by construction, $\hat{e}_{4}^{*}$ tends to $\hat{e}_{4}^{+}$at $\xi=+\infty$.

The critical points $\left(e_{3}^{+}(\lambda, \varepsilon), 1\right)$ and $\left(e_{2}^{+}(\lambda, \varepsilon), 1\right)$ have stable manifolds of (real) dimensions 3 and 5, respectively, since the matrix $d_{\hat{y}} \hat{a}$ at the first critical point has one eigenvalue with negative real part and two with positive real part, while this situation is reversed for the second critical point. Hence in the slice $\tau \equiv 1$ the stable subspaces are of (complex) dimension 1 and 2 , respectively. The $\tau^{\prime}$ equation adds one (real) stable direction to each. Let $\left(\hat{e}_{i}^{*}(\xi, \lambda, \varepsilon), \tau(\xi)\right)$ be any solution of $(5.1)$ in the stable manifold of $\left(e_{i}^{+}(\lambda), 1\right)$ such that $\tau(\xi)<1$, $i=2$ and 3 , and define $e_{i}^{*}=\tilde{e}_{i}^{*}$. By construction, $\hat{e}_{i}^{*}$ tends to $\hat{e}_{i}^{+}(\lambda, \varepsilon)$ as $\xi \rightarrow+\infty$.

The 3-plane bundle $\psi^{+}$over $\mathbb{R} \times K$ is defined to be

$$
\psi^{+}=\operatorname{span}\left\{e_{2}^{*}, e_{3}^{*}, e_{4}^{*}\right\} .
$$

Every solution in $\psi^{+}$can be expressed as

$$
y(\xi)=\sum_{i=2}^{4} \alpha_{i} e_{i}^{*}(\xi, \lambda, \varepsilon) \quad\left(\alpha_{i} \in \mathbb{C}\right) .
$$

Each $e_{i}^{*}$ grows/decays as $\xi \rightarrow+\infty$ at the rate $\exp \left(\mu_{i}^{+}(\lambda, \varepsilon) \xi\right)$; since $\operatorname{Re} \mu_{4}<$ $\operatorname{Re} \mu_{3}<0<\operatorname{Re} \mu_{2}$ it follows that the behavior of $y(\xi)$ at $\xi=+\infty$ is determined by the smallest index $i_{0}$ for which $\alpha_{i_{0}} \neq 0$; in particular, $\hat{y}(\xi)$ tends to 
$\hat{e}_{i_{0}}^{+}(\lambda, \varepsilon)$ as $\xi \rightarrow+\infty$. Thus every solution in $\psi^{+}$satisfies (b) in the statement of the theorem.

Next, consider the 2-plane bundle $\varphi^{-}$whose defining conditions are at $\xi=$ $-\infty$. Since the (complex) dimensions of $\varphi^{-}$and $\psi^{+}$add up to 5 and their fibers are in $\mathbb{C}^{4}$ they must intersect nontrivially. Moreover, by Corollary 4.5 to the elephant trunk lemma, the solution $\hat{e}_{1}(\xi, \lambda, \varepsilon)$ in $\varphi^{-}$tends to $\hat{e}_{1}^{+}(\lambda, \varepsilon)$ as $\xi \rightarrow+\infty$, whereas by condition (b) in the lemma, the 3-plane $\psi^{+}$near $\xi=+\infty$ tends (in the topology of $G_{3,4}$ ) to the plane spanned by $e_{i}^{+}(\lambda, \varepsilon)$, $i=2,3,4$. Since $e_{1}^{+}(\lambda, \varepsilon)$ is transverse to this 3-plane it follows that $\psi^{+}$and $\varphi^{-}$intersect transversely. We define $\hat{e}_{2}(\xi, \lambda, \varepsilon)$ to be the unique solution of (4.1) such that $\pi^{-1} \hat{e}_{2} \in \varphi^{-} \cap \psi^{+}$. By virtue of their behavior as $\xi \rightarrow+\infty$, $\hat{e}_{1}$ and $\hat{e}_{2}$ are distinct solutions of (4.1). Since the defining condition for $\hat{e}_{1}$, namely $\hat{e}_{1} \rightarrow \hat{e}_{1}^{-}(\lambda, \varepsilon)$ as $\xi \rightarrow-\infty$, uniquely determines $\hat{e}_{1}$ it follows that $\hat{e}_{2}$ must have an $\alpha$ limit set distinct from $\hat{e}_{1}^{-}(\lambda, \varepsilon)$. However $\pi^{-1} \hat{e}_{2}$ lies in $\varphi^{-}$so that the only other possible limit for $\hat{e}_{2}$ at $-\infty$ is $\hat{e}_{2}^{-}(\lambda, \varepsilon)$. Hence $\hat{e}_{2}$ satisfies (a) in the statement of the theorem.

We can sharpen (b) in Theorem 5.1 with a further application of the elephant trunk lemma.

Corollary 5.2. With hypotheses as in Theorem 5.1,

$$
\lim _{\xi \rightarrow+\infty} \hat{e}_{2}(\xi, \lambda, \varepsilon) \in\left\{\hat{e}_{2}^{+}(\lambda, \varepsilon), \hat{e}_{3}^{+}(\lambda, \varepsilon)\right\}
$$

i.e., $e_{2}$ is asymptotic to the slow subspace of $a^{+}(\lambda, \varepsilon)$ as $\xi \rightarrow+\infty$.

Proof. If the assertion were false it would follow that $\hat{e}_{2}(\xi, \lambda, \varepsilon)$ is asymptotic to $\hat{e}_{4}^{+}(\lambda, \varepsilon)$ as $\xi \rightarrow+\infty$. Let $\widehat{\Omega}^{s}$ be the negatively invariant set obtained from $\widehat{\Omega}^{u}$ of the elephant trunk theorem after a time reversal. Near the slow manifolds $\widehat{\Omega}^{s}$ is a uniform neighborhood of the fast stable eigenvector of $a(\xi, \lambda, \varepsilon)$, which we denote by $f_{4}(\xi, \lambda, \varepsilon)$. Since $f_{4}(\xi, \lambda, \varepsilon)$ tends to $e_{4}^{+}(\lambda, \varepsilon)$ as $\xi \rightarrow+\infty$ it follows from the above that $\left(\hat{e}_{2}(\xi, \lambda, \varepsilon), \xi\right)$ lies in $\widehat{\Omega}^{s}$ near $\xi=+\infty$. It would then follow from the elephant trunk lemma that $\hat{e}_{2}$ tends to $\hat{e}_{4}^{-}$as $\xi \rightarrow-\infty$, a contradiction to (a) of Theorem 5.1.

B. Approximation of $\hat{e}_{2}$-statement of the main result. In this section we formulate a theorem which characterizes the behavior of $\hat{e}_{2}$ as $\varepsilon \rightarrow 0$. It will be convenient to change to the slow scaling by considering the solution of $(3.3)_{s}$,

$$
E_{2}(\zeta, \lambda, \varepsilon)=e_{2}(\zeta / \varepsilon, \lambda, \varepsilon) .
$$

As usual we normalize the underlying wave by requiring that $U_{1}(0, \varepsilon)=h$ for all $\varepsilon \geq 0$; the transition layer then occurs in an $\mathscr{O}(\varepsilon)$ neighborhood of $\zeta=0$.

Roughly stated, the next theorem states that $\widehat{E}_{2}(\zeta, \lambda, \varepsilon)$ is approximated for small $\varepsilon>0$ by a solution $\widehat{E}_{2 R}(\zeta, \lambda)$ of the projectivization of the slow reduced system $(3.9)_{s R}$. This solution is obtained by gluing certain solution 
segments $E_{2 R}(\zeta, \lambda)$ of $(3.9)_{S R}$ together at $\zeta=0$ so that their 2-components are continuous across $\zeta=0$. The 1-components of $E_{2 R}$ are determined by the algebraic relations in $(3.9)_{S R}$; it follows that the $P_{1}$-component of $E_{2 R}$ is discontinuous at $\zeta=0$. The approximation will therefore fail to be uniform near $\zeta=0$. However, the approximation is uniform for the two-components when considered separately. This suggests introducing certain projection and inclusion maps between $\mathbb{C}^{2}$ and $\mathbb{C}^{4}$. Given solutions $Y$ of $(3.3)_{s}$ and $Y_{R}$ of $(3.9)_{s R}$ define $\Pi_{2}: \mathbb{C}^{4} \rightarrow \mathbb{C}^{2}$ to be projection onto the 2-components and introduce the variables $Z, Z_{R} \in \mathbb{C}^{2}$ :

$$
\begin{aligned}
Z & =\Pi_{2}(Y)=\left(P_{2}, Q_{2}\right)^{t}, \\
Z_{R} & =\Pi_{2}\left(Y_{R}\right)=\left(P_{2 R}, Q_{2 R}\right)^{t} .
\end{aligned}
$$

It will also be convenient to define a certain inclusion map $i: \mathbb{C}^{2} \rightarrow \mathbb{C}^{4}$. To this end let

$$
\begin{gathered}
H(\zeta, \lambda, \varepsilon)=\frac{A_{12}(\zeta, \varepsilon)}{\lambda-A_{11}(\zeta, \lambda)}, \quad H_{R}(\zeta, \lambda)=\frac{A_{12}^{R}(\zeta)}{\lambda-A_{11}^{R}(\zeta)}, \\
\Gamma=P_{1}-H(\zeta, \lambda, \varepsilon) P_{2}
\end{gathered}
$$

note that $H_{R}$ is the coefficient of $P_{2}$ in the algebraic equation in $(3.9)_{s R}$; for small $\varepsilon, \Gamma$ therefore measures how far $Y$ is from solving this equation. Define inclusion maps $i(\zeta, \varepsilon)$ and $i_{R}(\zeta)$ by

$$
\begin{aligned}
& i(\zeta, \lambda, \varepsilon) Z=\left(H(\zeta, \lambda, \varepsilon) P_{2}, 0, P_{2}, Q_{2}\right)^{t}, \\
& i_{R}(\zeta, \lambda) Z_{R}=\left(H_{R}(\zeta, \lambda) P_{2 R}, 0, P_{2 R}, Q_{2 R}\right)^{t} .
\end{aligned}
$$

The equations for the 2-components $Z=\Pi_{2} Y$ and $Z_{R}=\Pi_{2} Y_{R}$ of solutions of $(3.3)_{s},(3.9)_{s R}$ can then be expressed as

$$
\begin{gathered}
\dot{Z}=B(\zeta, \lambda, \varepsilon) Z+G \Gamma \\
\dot{Z}_{R}=B_{R}(\zeta, \lambda) Z_{R},
\end{gathered}
$$

where

$$
\begin{gathered}
B(\zeta, \lambda, \varepsilon)=\left(\begin{array}{cc}
0 & 1 \\
C(\zeta, \lambda, \varepsilon) & -\varepsilon \theta
\end{array}\right), \quad G=\left(\begin{array}{c}
0 \\
-A_{21}(\zeta, \varepsilon)
\end{array}\right) \\
C(\zeta, \lambda, \varepsilon)=-A_{21}(\zeta, \varepsilon) H(\zeta, \lambda, \varepsilon)-A_{22}+\lambda,
\end{gathered}
$$

and

$$
\begin{gathered}
B_{R}(\zeta, \lambda)=\left(\begin{array}{cc}
0 & 1 \\
C_{R}(\zeta, \lambda) & 0
\end{array}\right), \\
C_{R}(\zeta, \lambda)=-A_{21}^{R}(\zeta) H_{R}(\zeta, \lambda)-A_{22}^{R}(\zeta)+\lambda .
\end{gathered}
$$

The equations $(5.4)_{R}$ are equivalent to the equations for the 2-components in $(3.9)_{s R}$ and $Z_{R}(\zeta)$ is a solution of $(5.4)_{R}$ if and only if $i_{R}(\zeta, \lambda) Z_{R}(\zeta)$ is a solution of $(3.9)_{S R}$. 
Definition. $\zeta \in \Omega$ is an eigenvalue of the slow reduced system (3.9) ${ }_{s R}$ if there exists a nontrivial, uniformly bounded solution $Z_{R}(\zeta, \lambda)$ of $(5.3)_{R}$ which is continuous across $\zeta=0$.

We remark that for $\lambda \in \Omega,(5.4)_{R}$ has a saddle at $\zeta= \pm \infty$, so that an eigenfunction must decay to zero at $\zeta= \pm \infty$. Note also that $(5.4)_{R}$ is equivalent to the following second order eigenvalue problem,

$$
\begin{gathered}
\ddot{P}_{2}+C_{R}(\zeta, \lambda) P_{2}=0 \\
C_{R}(\zeta, \lambda)=\frac{-A_{12}^{R}(\zeta) A_{21}(\zeta)}{\lambda-A_{11}^{R}(\zeta)}-A_{22}^{R}(\zeta)+\lambda,
\end{gathered}
$$

and that the eigenvalue parameter $\lambda$ enters into the equation in a nonlinear manner. We assume for the moment that the spectrum of this problem is known; it will be explicitly characterized later in the paper (see $\S \mathrm{VI}$ ).

It will be convenient to consider the projectivization of $(5.4)_{R}$ on $\mathbb{C} P^{1}$, which we express as

$$
\widehat{Z}^{\bullet}=\widehat{B}_{R}(\widehat{Z}, \zeta, \lambda) .
$$

The following approximation theorem will be concerned with two particular solutions $\widehat{Z}_{2 R}(\zeta, \lambda)$ and $\hat{Z}_{*}(\zeta, \lambda)$ of $(5.5)_{R}$. First, $\hat{Z}_{2 R}(\zeta, \lambda)$ is defined to be the (unique) solution of $(5.5)_{R}$ which, as $\zeta \rightarrow-\infty$, tends to the image in $\mathbb{C} P^{1}$ of the unstable eigenvector $\left(1, C_{R}(-\infty, \lambda)^{1 / 2}\right)$ of $B_{R}(-\infty, \lambda)$. The existence and uniqueness of $\hat{Z}_{2 R}$ follows from general results appearing in [AGJ]. The second solution $\hat{Z}_{*}(\zeta, \lambda)$ is defined as follows. Let $Z=\Pi_{2} E_{2}$, where $E_{2}(\zeta, \lambda, \varepsilon)$ is any nontrivial representative of $\widehat{E}_{2}(\zeta, \lambda, \varepsilon)$. By passing to suitable subsequences $\varepsilon_{n} \rightarrow 0$ it can be assumed that the limits

$$
\widehat{Z}\left( \pm 1, \lambda, \varepsilon_{n}\right)=\widehat{Z}^{ \pm}
$$

both exist as $\varepsilon \rightarrow 0$. Let $\widehat{Z}^{ \pm}(\zeta, \lambda)$ be the solution of $(5.5)_{R}$ with data

$$
\widehat{Z}^{ \pm}( \pm 1, \lambda)=\widehat{Z}^{ \pm}
$$

finally, define $\hat{Z}_{*}(\zeta, \lambda)$ by

$$
\hat{Z}_{*}(\zeta, \lambda)= \begin{cases}\hat{Z}^{-}(\zeta, \lambda), & \zeta<0 \\ \widehat{Z}^{+}(\zeta, \lambda), & \zeta>0\end{cases}
$$

If $Z_{2 R}$ and $Z_{*}$ are any representatives in $\pi^{-1} \hat{Z}_{2 R}$ and $\pi^{-1} \hat{Z}_{*}$, where $\pi: \mathbb{C}^{2} \rightarrow$ $\mathbb{C} P^{1}$ is the projection map, define solutions $E_{2 R}$ and $E_{*}$ of $(3.9)_{s R}$ by

$$
E_{2 R}(\zeta, \lambda)=i_{R}(\zeta, \lambda) Z_{2 R}(\zeta, \lambda), \quad E_{*}(\zeta, \lambda)=i_{R}(\zeta, \lambda) Z_{*}(\zeta, \lambda),
$$

where $i_{R}$ is the map in $(5.2 \mathrm{c})$. We can now state the main result of this section. 
Theorem 5.3. With hypotheses as in Theorem 5.1 there exists $\bar{\varepsilon}>0$ such that for $0<\varepsilon \leq \bar{\varepsilon}$ the following are true:

(a) $\lim _{\varepsilon \rightarrow 0} \widehat{E}_{2}(\zeta, \lambda, \varepsilon)=\widehat{E}_{*}(\zeta, \lambda)$ uniformly for $a \leq|\zeta| \leq A$, for each $a, A$ with $0<a<1<A$.

(b) $Z_{*}(\zeta, \lambda)$ is continuous across $\zeta=0$.

(c) Suppose that $\lambda \in \Omega$ is not an eigenvalue of $(3.9)_{s R}$. Then $\widehat{Z}_{*}(\zeta, \lambda) \equiv$ $\widehat{Z}_{2 R}(\zeta, \lambda)$, and for $\varepsilon \leq \bar{\varepsilon}$,

$$
\lim _{\zeta \rightarrow+\infty} \widehat{E}_{2}(\zeta, \lambda, \varepsilon)=\hat{e}_{2}^{+}(\lambda, \varepsilon) .
$$

Moreover, the limit is uniform in $\lambda$ for $\lambda \in K$.

The proof of Theorem 5.3 will require some preliminary estimates. The principal estimate asserts that $\widehat{E}_{2}$ remains uniformly near the "slow subbundle", which is defined in subsection $D$ below. It is this fact that also forces the continuity of $\hat{Z}_{*}$ across $\zeta=0$. The main aim of the theorem is to characterize the behavior of $\widehat{E}_{2}$ at $\zeta=+\infty$. Since $\widehat{E}_{2}$ remains near a hyperbolic set rather than an attractor in $\mathbb{C} P^{3}$, the elephant trunk lemma is not available. The idea is to use an "elephant trunk" type estimate inside the slow subbundle.

C. Estimates. In this section we shall use the estimates of $\S$ IIE for the underlying waves $x(\xi, \varepsilon)=X(\xi, \varepsilon)$ to approximate the coefficient matrix $a(\xi, \lambda, \varepsilon)$ of the linearized equations. There are two cases to consider which are determined by whether the wave is near the slow manifolds or in the transition layer.

Case 1. Suppose that $x\left(\xi_{n}, \varepsilon_{n}\right)$ tends to $\bar{x}$ as $\varepsilon_{n} \rightarrow 0$ for some uniformly bounded sequence $\left\{\xi_{n}\right\}$. By passing to a subsequence it can be assumed that $\xi_{n}$ tends to a finite limit $\bar{\xi}$. Let $x_{R}(\xi)$ be the solution of the fast reduced system $(2.3)_{f R}$,

$$
x_{R}^{\prime}=n\left(x_{R}, 0\right), \quad x_{R}(\bar{\xi})=\bar{x} .
$$

Then $x_{R}(\xi)$ is the connecting orbit in (2.4) with $u_{2} \equiv \alpha$ and $\theta=\Theta(\alpha)$, and

$$
\begin{array}{ll}
\left|x\left(\xi, \varepsilon_{n}\right)-x_{R}(\xi)\right| \rightarrow 0 & \text { uniformly as } \varepsilon_{n} \rightarrow 0 \\
& \text { on bounded } \xi \text {-intervals about } \bar{\xi} .
\end{array}
$$

This is an immediate consequence of Corollary 2.2, which shows that $x(\xi, \varepsilon)$ lies in $n(\delta)$ for all sufficiently small $\varepsilon$. Since $\bar{\xi}$ is finite and $\delta>0$ is arbitrary it follows that $x_{R}(\xi)$ lies in $n_{F}(\delta)$ for all $\delta>0$; the result follows by applying Corollary 2.2 and Gronwall's inequality to the equation satisfied by $x(\xi, \varepsilon)-$ $x_{R}(\xi)$.

As a consequence of (5.6) we obtain under similar hypotheses as above that

$$
\begin{array}{ll}
\left|a\left(\xi, \lambda, \varepsilon_{n}\right)-a_{R}(\xi, \lambda)\right| \rightarrow 0 & \text { uniformly as } \varepsilon_{n} \rightarrow 0 \\
& \text { on bounded } \xi \text {-intervals about } \bar{\xi} .
\end{array}
$$

Case 2. Suppose that $X\left(\zeta_{n}, \varepsilon_{n}\right)$ converges to $\bar{x}$ as $\varepsilon_{n} \rightarrow 0$ and that $\zeta_{n}=\varepsilon_{n} \xi_{n}$ converges to a limit $\bar{\zeta}$, where either $\bar{\zeta}$ is finite or $\bar{\zeta}= \pm \infty$. If $\bar{\zeta}=0$, suppose 
in addition that $\left\{\xi_{n}\right\}$ is divergent, i.e., this sequence tends either to $-\infty$ or to $+\infty$. Let $\zeta_{n}+\sigma=\varepsilon_{n}\left(\xi_{n}+s\right)$ and define $x_{n}\left(s, \varepsilon_{n}\right)=x\left(\xi_{n}+s, \varepsilon_{n}\right), X_{n}\left(\sigma, \varepsilon_{n}\right)=$ $X\left(\zeta_{n}+\sigma, \varepsilon_{n}\right)$. Then $\bar{x}$ lies in the slow manifolds $S_{L} \cup S_{R}$ and

$$
\begin{array}{ll}
\left|x_{n}\left(s, \varepsilon_{n}\right)-\bar{x}\right| \rightarrow 0 & \text { uniformly for bounded } s \text {-intervals } \\
& \text { about } s=0 \text { as } \varepsilon_{n} \rightarrow 0 .
\end{array}
$$

The proof of (5.8) is also in immediate consequence of Corollary 2.2 and Gronwall's inequality. This can again be used to approximate the coefficient matrix $a(\xi, \lambda, \varepsilon)$ along the slow manifolds. In particular, let $a_{R}(\lambda)$ be the coefficient matrix of the fast reduced equations $(3.9)_{f R}$ wherein the coefficients $a_{i j}^{R}(\xi)$ are replaced by the (constant) Jacobian of $\left(u_{1} f_{1}, u_{2} f_{2}\right)$ evaluated at the $u$ components of $\bar{x}$. It then follows from (5.8) that

$$
\begin{aligned}
&\left|a\left(\xi_{n}+s, \lambda, \varepsilon_{n}\right)-a_{R}(\lambda)\right| \rightarrow 0 \quad \text { as } \varepsilon_{n} \rightarrow 0 \text { uniformly on bounded } \\
& s \text {-intervals about } s=0 .
\end{aligned}
$$

D. Hyperbolicity of the slow bundle. It will be convenient to introduce an approximate slow bundle for the linearized equations. To this end, fix $a>0$; for $|\xi| \geq a / \varepsilon$ the wave $x(\xi, \varepsilon)$ lies near $S_{L} \cup S_{R}$ for small $\varepsilon$ so that by Remark 3.2, the coefficient matrix $a(\xi, \lambda, \varepsilon)$ of $(3.3)_{f}$ will have eigenvalues $\mu_{i}(\xi, \lambda, \varepsilon)$ which satisfy $(3.7 \mathrm{a})$ for $i=1,4$. However, the eigenvalues $\mu_{i}(\xi, \lambda, \varepsilon), i=2,3$, can coalesce for certain $\xi$ although they will still be of or$\operatorname{der} \varepsilon$. By a general result (see Kato $[\mathrm{K}]$ ) we may select smooth $f_{i}(\xi, \lambda, \varepsilon) \in \mathbb{C}^{4}$ such that $f_{2}, f_{3}$ at $(\xi, \lambda, \varepsilon)$ span the (generalized) eigenspace of $a(\xi, \lambda, \varepsilon)$ associated with $\mu_{2}(\xi, \lambda, \varepsilon)$ and $\mu_{3}(\xi, \lambda, \varepsilon)$. Normalize $f_{i}$ so that $\left|f_{i}\right|_{\infty}=1$ for all $(\xi, \lambda, \varepsilon)$.

Definition. The slow subbundle $\sigma_{2}$ of $(3.3)_{f}$ is

$$
\sigma_{s}(\xi, \lambda, \varepsilon)=\operatorname{span}\left\{f_{2}(\xi, \lambda, \varepsilon), f_{3}(\xi, \lambda, \varepsilon)\right\}
$$

the base space of $\sigma_{s}$ is $|\xi| \geq a / \varepsilon, \lambda \in \Omega$, and $0<\varepsilon \leq \bar{\varepsilon}$, where $\bar{\varepsilon}$ is small enough so that Remark 3.2 is valid.

We shall approximate solutions of $(3.3)_{f}$ by considering the frozen system and its projectivization

$$
\begin{gathered}
\frac{d y}{d s}=a(\gamma, \lambda, \varepsilon) y, \\
\frac{d \hat{y}}{d s}=\hat{a}(\hat{y}, \gamma, \lambda, \varepsilon) .
\end{gathered}
$$

It will be convenient in the following to fix a metric $\rho$ on $\mathbb{C} P^{3}$ so that for a given set $\widehat{S} \subset \mathbb{C} P^{3}$ we may define a $\delta$-neighborhood by

$$
N_{\delta}(\widehat{S})=\left\{\hat{y} \in \mathbb{C} P^{3}: \rho(\hat{s}, \hat{y})<\delta \text { for some } \hat{s} \in \widehat{S}\right\} \text {. }
$$

Lemma 5.4. Fix $\delta>0$ and $a>0$; there exist $\bar{\varepsilon}>0$ and $T>0$ depending only on $\delta$ and a such that for $0<\varepsilon \leq \bar{\varepsilon}$ and $|\gamma| \geq a / \varepsilon$, every solution $\hat{y}(s)$ of $(5.11)_{\gamma}$ satisfies at least one of the following:

(i) $\hat{y}(0) \in N_{\delta}\left(\hat{\sigma}_{s}(\gamma, \lambda, \varepsilon)\right)$, 
(ii) $\hat{y}(T) \in N_{\delta}\left(\hat{f}_{1}(\gamma, \lambda, \varepsilon)\right)$,

(iii) $\hat{y}(-T) \in N_{\delta}\left(\hat{f}_{4}(\gamma, \lambda, \varepsilon)\right)$.

Proof. Let $\hat{y}(s)$ be a solution of (5.11), which does not satisfy (i). If $y(s)$ is an associated solution of $(5.10)_{\gamma}$, then $y(s)$ can be expressed as

$$
y(s)=\sum_{i=1}^{4} g_{i} f_{i}(\gamma, \lambda, \varepsilon) \exp \left(s \mu_{i}(\gamma, \lambda, \varepsilon)\right) ;
$$

for simplicity we have assumed that $a(\gamma, \lambda, \varepsilon)$ has distinct eigenvalues. If this is not the case, the proof is similar. We shall choose the particular element of $\pi^{-1} \hat{y}(s)$ such that $|y(0)|=1$ in the sup norm; since $\left|f_{i}\right|=1$ it follows that $\left|g_{i}\right| \leq 1$ for $1 \leq i \leq 4$.

Since $\hat{y}(0)$ does not satisfy (i) it is clear that (ii) or (iii) hold for some $T$; the point is to verify the choice of $T$ is uniform for such $\hat{y}(0), 0<\varepsilon \leq \bar{\varepsilon}$, and $|\gamma| \geq a / \bar{\varepsilon}$. From the above, there exists a constant $K>0$ depending only on the metric $\rho$ such that

$$
\max \left\{\left|g_{1}\right|,\left|g_{4}\right|\right\} \geq K \delta \text {. }
$$

We will show that if $\left|g_{1}\right| \geq K \delta$ then (ii) holds. From (5.12) we have that

$$
y(T)=e^{\mu_{1} T}\left[g_{1} f_{1}+R(T)\right],
$$

where

$$
R(T)=\sum_{i=1}^{4} g_{i} f_{i} e^{T\left(\mu_{i}-\mu_{1}\right)}
$$

For $0<\varepsilon \leq \bar{\varepsilon}$ and $|\gamma| \geq a / \bar{\varepsilon}$, it follows from (3.7a) that there exists $\alpha>0$ depending only on $\bar{\varepsilon}$ and $a$ such that $\operatorname{Re}\left(\mu_{i}-\mu_{1}\right)<-\alpha$ for $i=2,3,4$. Since $\left|g_{i}\right| \leq 1$ and $\left|f_{i}\right|=1$ for all $i$ it follows from the above that

$$
|R(T)|<3 e^{-\alpha T}
$$

From (5.14) we obtain $T>0$ depending only on $\alpha, \delta, a$, and the metric $\rho$ such that

$$
\hat{y}(\Gamma) \in N_{\delta}\left(\hat{f}_{1}(\gamma, \lambda, \varepsilon)\right)
$$

for $|\gamma| \geq a / \varepsilon, 0<\varepsilon \leq \bar{\varepsilon}$.

The proof that $\left|g_{4}\right| \geq K \delta$ implies (iii) is similar.

It will be convenient to use the slow scaling at this point. For $|\zeta| \geq a$ and $0<\varepsilon \leq \bar{\varepsilon}$, let

$$
\begin{gathered}
F_{i}(\zeta, \lambda, \varepsilon)=f_{i}(\zeta / \varepsilon, \lambda, \varepsilon), \quad 1 \leq i \leq r, \\
\Sigma_{s}(\zeta, \lambda, \varepsilon)=\sigma_{s}(\zeta / \varepsilon, \lambda, \varepsilon) .
\end{gathered}
$$

The slow projectivized equations associated with $(3.3)_{s}$ will be denoted by

$$
\widehat{Y}^{\bullet}=\widehat{A}(\widehat{Y}, \zeta, \lambda, \varepsilon) \text {. }
$$


We next prove a lemma for solutions $\hat{Y}(\zeta)$ of the nonautonomous equations (5.15). First we need to set $\delta$ in the previous lemma. To this end let $\widehat{\Omega}^{s}$ and $\widehat{\Omega}^{u}$ be the sets constructed in the elephant trunk lemma; let $\widehat{\Omega}^{s}(\xi)$ and $\widehat{\Omega}^{u}(\xi)$ denote the projections of $\widehat{\Omega}^{s}$ and $\widehat{\Omega}^{u}$ on the slice $\mathbb{C} P^{3} \times\{\xi\}$. Set $\bar{\varepsilon}>0$ so small that $a / \bar{\varepsilon} \geq \xi_{R},\left|\xi_{L}\right|$. It follows that there exists $\delta_{1}>0$ such that for $0<\varepsilon \leq \bar{\varepsilon}$,

$$
\begin{array}{ll}
N_{\delta_{1}}\left(\hat{f}_{1}(\xi, \lambda, \varepsilon)\right) \subset \hat{\Omega}^{u}(\xi) & \text { for }|\xi| \geq a / \varepsilon, \\
N_{\delta_{1}}\left(\hat{f}_{4}(\xi, \lambda, \varepsilon)\right) \subset \widehat{\Omega}^{s}(\xi) \text { for }|\xi| \geq a / \varepsilon .
\end{array}
$$

In particular, $\delta_{1}$ depends only on $a$, the defining parameters $d_{L}, d_{R}, \eta_{L}, \eta_{R}$ for $\widehat{\Omega}^{u}$ and $\widehat{\Omega}^{s}$, and on the metric $\rho$ on $\mathbb{C} P^{3}$.

Lemma 5.5. Set $0<\delta<\delta_{1}$ as in (5.16) and $a>0$. Suppose that $\widehat{Y}(\zeta, \lambda, \varepsilon)$ is a solution of (5.15) such that for each sufficiently small $\varepsilon>0$ there exists $A(\varepsilon)>a$ with the property that

$$
\widehat{Y}(\zeta, \lambda, \varepsilon) \in N_{\delta}\left(\Sigma_{s}(\zeta, \lambda, \varepsilon)\right) \text { for }|\zeta| \geq A(\varepsilon) .
$$

Suppose also that the elephant trunk lemma is valid at $(\lambda, \varepsilon)$. Then there exists $\bar{\varepsilon}>0$ such that for $0<\varepsilon \leq \bar{\varepsilon}$ and $|\zeta| \geq a$ we have that

$$
\widehat{Y}(\zeta, \lambda, \varepsilon) \in N_{\delta}\left(\Sigma_{s}(\zeta, \lambda, \varepsilon)\right) .
$$

Proof. Suppose that the lemma is false; there would then exist sequences $\left|\zeta_{n}\right| \geq$ $a$ and $\varepsilon_{n} \rightarrow 0$ such that (5.17) fails to hold for all $n$. By passing to subsequences it can be assumed that the following sequences are convergent;

$$
\begin{gathered}
\zeta_{n} \rightarrow \bar{\zeta}, \quad \text { where } a \leq|\bar{\zeta}| \leq \infty, \\
X\left(\zeta_{n}, \varepsilon_{n}\right) \rightarrow \bar{x} \in S_{L} \cup S_{R}, \\
\widehat{Y}\left(\zeta_{n}, \lambda, \varepsilon_{n}\right)=\widehat{Y}_{n} \rightarrow \hat{Y}_{*} \text { in } \mathbb{C} P^{3} .
\end{gathered}
$$

Let $s=\left(\zeta-\zeta_{n}\right) / \varepsilon_{n}$ and $\gamma_{n}=\zeta_{n} / \varepsilon_{n}$; change variables from $\zeta$ to $s$ by defining

$$
\hat{y}(\xi, \lambda, \varepsilon)=\hat{Y}(\xi, \lambda, \varepsilon), \quad \hat{z}\left(s, \lambda, \varepsilon_{n}\right)=\hat{Y}\left(s \varepsilon_{n}+\zeta_{n}, \lambda, \varepsilon_{n}\right) .
$$

Then $\hat{z}$ satisfies the equation

$$
\frac{d \hat{z}}{d s}=\hat{a}\left(\hat{z}, \gamma_{n}+s, \lambda, \varepsilon_{n}\right), \quad \hat{z}(0)=\widehat{Y}_{n} .
$$

Next, let $\hat{z}_{*}(s, \lambda, n)$ be the solution of the frozen system

$$
\frac{d \hat{z}_{*}}{d s}=\hat{a}\left(\hat{z}_{*}, \gamma_{n}, \lambda, \varepsilon_{n}\right), \quad \hat{z}_{*}(0, \lambda, n)=\hat{Y}_{n} .
$$

By hypothesis, $\widehat{Y}_{n} \notin N_{\delta}\left(\hat{\sigma}_{s}\left(\gamma_{n}, \lambda, \varepsilon_{n}\right)\right)$ for all $n$; it follows from Lemma 5.4 that at least one of

(a) $\hat{Z}_{*}(T, \lambda, n) \in N_{\delta}\left(\hat{f}_{1}\left(\gamma_{n}, \lambda, \varepsilon_{n}\right)\right)$,

(b) $\hat{Z}_{*}(-T, \lambda, n) \in N_{\delta}\left(\hat{f}_{4}\left(\gamma_{n}, \lambda, \varepsilon_{n}\right)\right)$ 
hold, with $T$ as in the statement of Lemma 5.4. Now set $\delta_{2}$ such that $\delta<\delta_{2}<\delta_{1}$; it follows from equation (5.9) that the vector fields $\hat{a}\left(\hat{z}_{*}, \gamma_{n}, \lambda, \varepsilon_{n}\right)$ and $\hat{a}\left(\hat{z}_{*}, \gamma_{n}+s, \lambda, \varepsilon_{n}\right)$ converge uniformly to $\hat{a}_{R}\left(\hat{z}_{*}, \lambda\right)$ for $\hat{z} \in \mathbb{C} P^{3}$, where $a_{R}(\lambda)_{*}$ is the matrix defined above (5.9). The convergence is uniform on the interval $|s| \leq T$. Furthermore, it also follows from (5.9) that the vectors $f_{i}\left(\gamma_{n}, \lambda, \varepsilon_{n}\right)$ and $f_{i}\left(\gamma_{n}+s, \lambda, \varepsilon_{n}\right)$ converge uniformly as $\varepsilon_{n} \rightarrow 0$ for $|s| \leq T$, $1 \leq i \leq 4$. It then follows from the above and standard continuous dependence theorems for flows that $\hat{z}_{*}(s, \lambda, n)$ and $\hat{z}\left(s, \lambda, \varepsilon_{n}\right)$ converge uniformly on $|s| \leq T$ as $\varepsilon_{n} \rightarrow 0$. By (a) or (b) and the preceding remarks it follows that at least one of the following hold:

$$
\begin{gathered}
\hat{z}\left(T, \lambda, \varepsilon_{n}\right)=\hat{Y}\left(\zeta_{n}+\varepsilon_{n} T, \lambda, \varepsilon_{n}\right) \in N_{\delta_{2}}\left(\hat{f}_{1}\left(\gamma_{n}+T, \lambda, \varepsilon_{n}\right)\right), \\
\hat{z}\left(-T, \lambda, \varepsilon_{n}\right)=\hat{Y}\left(\zeta_{n}-\varepsilon_{n} T, \lambda, \varepsilon_{n}\right) \in N_{\delta_{2}}\left(\hat{f}_{4}\left(\gamma_{n}-T, \lambda, \varepsilon_{n}\right)\right) .
\end{gathered}
$$

Since $\delta<\delta_{2}<\delta_{1}$, it then follows from (5.16) that $\widehat{Y}$ enters at least one of $\widehat{\Omega}^{u}$ or $\widehat{\Omega}^{s}$ in at least one time direction. The elephant trunk lemma contradicts the postulated behavior of $\hat{Y}$ near $\zeta= \pm \infty$.

Corollary 5.6. With $a, \delta$, and $\bar{\varepsilon}$ as in Lemma 5.5, we have that

$$
\widehat{E}_{2}(\zeta, \lambda, \varepsilon) \in N_{\delta}\left(\widehat{\Sigma}_{s}(\zeta, \lambda, \varepsilon)\right)
$$

for $|\zeta| \geq a$ and $0<\varepsilon \leq \bar{\varepsilon}$.

By (a) of Theorem 5.1 and Corollary 5.2, $\widehat{E}_{2}$ satisfies the hypothesis of Lemma 5.5 for all sufficiently small $\varepsilon$ for some $A(\varepsilon)$ sufficiently large.

E. Bounds in the transition layer. In order to prove the continuity of $\hat{Z}_{*}(\zeta, \lambda)$ at $\zeta=0$ we shall require uniform bounds for a solution $E_{2}$ in $\pi^{-1} \widehat{E}_{2}$ near $\zeta=0$. To this end let $\widehat{Y}(\zeta, \lambda, \varepsilon)$ be a solution of (5.15). Given an element $Y \in \pi^{-1} \widehat{Y}$ we define two quantities

$$
\begin{aligned}
& M_{1}(\varepsilon, \lambda)=\max _{|\zeta| \leq 1}\left\{\left|P_{1}(\zeta, \lambda, \varepsilon)\right|,\left|Q_{1}(\zeta, \lambda, \varepsilon)\right|\right\}, \\
& M_{2}(\varepsilon, \lambda)=\max _{|\zeta| \leq 1}\left\{\left|P_{2}(\zeta, \lambda, \varepsilon)\right|,\left|Q_{2}(\zeta, \lambda, \varepsilon)\right|\right\} .
\end{aligned}
$$

For the moment, we regard $\lambda \in \Omega$ as fixed; replace $Y$ with the section $Y / M_{2}(\varepsilon, \lambda) \in \pi^{-1} \hat{Y}$. The 2-components of $Y$ then satisfy

$$
\left|P_{2}(\zeta, \lambda, \varepsilon)\right|,\left|Q_{2}(\zeta, \lambda, \varepsilon)\right| \leq 1 \quad \text { on }|\zeta| \leq 1
$$

for all $\varepsilon \in(0, \bar{\varepsilon}]$.

Lemma 5.7. Suppose that $(\lambda, \varepsilon)$ is such that the elephant trunk lemma is valid. Let $Y \in \pi^{-1} \widehat{Y}$ be selected so that (5.18) is valid for all $\varepsilon \in(0, \bar{\varepsilon}]$, where $\hat{Y}$ is a solution of (5.15). Suppose also that $\widehat{Y}$ satisfies the hypothesis of Lemma 5.5 at $\zeta= \pm \infty ;$ then there exists $\bar{M}_{1}$ such that

$$
\sup _{\substack{0<\varepsilon<\bar{\varepsilon} \\ \lambda \in \bar{K}}} M_{1}(\varepsilon, \lambda) \leq \bar{M}_{1}<\infty
$$


where $K$ is a curve as in Figure 3.1 which is disjoint from eigenvalues $\lambda$ of $(3.9)_{f}$.

Proof. If the lemma were false there would exist sequences $\varepsilon_{n}, \lambda_{n}$ with $\varepsilon_{n} \rightarrow 0$ such that $M_{1}\left(\varepsilon_{n}, \lambda_{n}\right) \rightarrow \infty$. By passing to a subsequence we may assume that $\lambda_{n} \rightarrow \lambda \in K$. Replace the solution $Y$ with $Y / M_{1}$ for each $n$ and relable this solution $Y\left(\zeta, \lambda_{n}, \varepsilon_{n}\right)$. It follows from (5.18) that $Y$ satisfies

$$
\begin{gathered}
\max _{|\zeta| \leq 1}\left\{\left|P_{2}\left(\zeta, \lambda_{n}, \varepsilon_{n}\right)\right|,\left|Q_{2}\left(\zeta, \lambda_{n}, \varepsilon_{n}\right)\right|\right\} \rightarrow 0 \text { as } n \rightarrow \infty, \\
\max _{|\zeta| \leq 1}\left\{\left|P_{1}\left(\zeta, \lambda_{n}, \varepsilon_{n}\right)\right|,\left|Q_{1}\left(\zeta, \lambda_{n}, \varepsilon_{n}\right)\right|\right\}=1, \quad \text { all } n .
\end{gathered}
$$

By passing to another subsequence we may assume that the maximum in $(5.19 b)$ occurs at $\zeta_{n}$, where $\zeta_{n}$ converges to a limit $\bar{\zeta} \in[-1,1]$. We may further assume that

$$
\begin{gathered}
P_{1}\left(\zeta_{n}, \lambda_{n}, \varepsilon_{n}\right), Q_{1}\left(\zeta_{n}, \lambda_{n}, \varepsilon_{n}\right) \rightarrow \bar{P}_{1}, \bar{Q}_{1} \quad \text { as } n \rightarrow \infty, \\
P_{2}\left(\zeta, \lambda_{n}, \varepsilon_{n}\right), Q_{2}\left(\zeta, \lambda_{n}, \varepsilon_{n}\right) \rightarrow 0,0 .
\end{gathered}
$$

Let $\zeta_{n}=\varepsilon_{n} \xi_{n}$; we distinguish three different cases.

Case 1: $\left|\zeta_{n}\right| \geq a$ for some $a>0$, and all $n$. It follows from Lemma 5.5 that given $\delta>0$ chosen as in the lemma, $\widehat{Y}$ satisfies (5.17) for all $|\zeta| \geq a$. However, from the limiting form $(3.7 \mathrm{~b})$ for $F_{2}(\zeta, \lambda, \varepsilon), F_{3}(\zeta, \lambda, \varepsilon)$, which span $\Sigma_{s}(\zeta, \lambda, \varepsilon)$, it is evident from (5.20) that $Y\left(\zeta_{n}, \lambda_{n}, \varepsilon_{n}\right)$ lies at a uniform distance $d$ from the projectivized slow subspace $\widehat{\Sigma}_{s}\left(\zeta_{n}, \lambda_{n}, \varepsilon_{n}\right)$ as $\varepsilon_{n} \rightarrow 0$. We arrive at a contradiction by choosing $\delta$ of Lemma 5.5 suitably small relative to $d$.

Case 2: $\zeta_{n} \rightarrow 0$ but $\xi_{n}$ diverges to $\pm \infty$. This can be handled similarly to the previous case because the limit

$$
\bar{x}=\lim X\left(\zeta_{n}, \varepsilon_{n}\right)
$$

still lies in $S_{L} \cup S_{R}$; if $\xi_{n} \rightarrow-\infty$ it is the left corner while if $\xi_{n} \rightarrow+\infty$ it is the right corner. In particular, the splitting (3.7a) is valid and the slow subspace $\Sigma\left(\zeta, \lambda_{n}, \varepsilon_{n}\right)$ will be well defined for all $|\zeta| \geq\left|\zeta_{n}\right|$ and for all sufficiently large $n$. Since the asymptotic form $(3.7 \mathrm{~b})$ for $F_{2}(\zeta, \lambda, \varepsilon)$ and $F_{3}(\zeta, \lambda, \varepsilon)$ will be valid as $\varepsilon_{n} \rightarrow 0$, it again follows that $\hat{Y}\left(\zeta_{n}, \lambda_{n}, \varepsilon_{n}\right)$ will be uniformly bounded away from $\widehat{\Sigma}_{s}\left(\zeta_{n}, \lambda_{n}, \varepsilon_{n}\right)$ as $\varepsilon_{n} \rightarrow 0$, and by changing variables from $\zeta$ to $s=\left(\zeta-\zeta_{n}\right) / \varepsilon_{n}$, as in the proof of Lemma 5.5 , we obtain a contradiction by using the frozen system and Lemma 5.4 on the uniform interval $|s| \leq T$.

Case 3: $\zeta_{n} \rightarrow 0$ and $\left|\xi_{n}\right|$ is uniformly bounded. In this case we are genuinely in the transition layer. By passing to a subsequence it can be assumed that $\xi_{n}$ converges to a finite limit $\bar{\xi}$. Let

$$
\bar{y}=\lim _{n \rightarrow \infty} Y\left(\zeta_{n}, \lambda_{n}, \varepsilon_{n}\right)=\left(\bar{P}_{1}, \bar{Q}_{1}, 0,0\right) ;
$$


by (5.19), $\max \left\{\left|\bar{P}_{1}\right|,\left|\bar{Q}_{1}\right|\right\}=1$. Also, let $y\left(\xi, \lambda_{n}, \varepsilon_{n}\right)$ be the associated solutions in the fast scaling and let $y_{R}(\xi, \lambda)$ be the solution of $(3.9)_{f}$ with

$$
y_{R}(\bar{\xi}, \lambda)=\bar{y} \text {. }
$$

We claim that there exists $\bar{s}>0$ which is independent of $n$ such that at least one of the following hold for all large $n$ :

(i) $\hat{y}\left(\bar{s}, \lambda_{n}, \varepsilon_{n}\right) \in \widehat{\Omega}^{u}(\bar{s})$,

(ii) $\hat{y}\left(-\bar{s}, \lambda_{n}, \varepsilon_{n}\right) \in \widehat{\Omega}^{s}(-\bar{s})$;

in either case, we will have then obtained the desired contradiction.

Let $a_{R}(\xi, \lambda)$ be the coefficient matrix of $(3.9)_{f R}$, let $e_{1 R C}^{+}(\lambda)$ be the fast unstable eigenvector of $a_{R}(+\infty, \lambda)$ (in the right corner), and let $e_{4 R C}^{-}(\lambda)$ be the fast stable eigenvector of $a_{R}(-\infty, \lambda)$ (in the left corner). Since $\lambda \in K$ is not an eigenvalue of $(3.9)_{f R}$ at least one of the following must be true:

(1) $\lim _{\xi \rightarrow+\infty} \hat{y}_{R}(\xi, \lambda)=\hat{e}_{1 R C}^{+}(\lambda)$,

(2) $\lim _{\xi \rightarrow-\infty} \hat{y}_{R}(\xi, \lambda)=\hat{e}_{4 R C}^{-}(\lambda)$.

We will show that (1) implies (i); the proof that (2) implies (ii) is similar and will be omitted.

For $\xi \geq \xi_{R}\left(d_{R}\right), \widehat{\Omega}^{u}(\xi)$ is a disk in $\mathbb{C} P^{3}$ of uniform radius $\eta_{R}$ about $\hat{f}_{1}(\xi, \lambda, \varepsilon)$ (measured in the norm $|\cdot|_{\xi}$ defined in $\S \mathrm{IV}$ ). Let $f_{1 R}(\xi, \lambda)$ be the fast unstable eigenvector of the matrix $a_{R}(\xi, \lambda)$. Since our parametrizations for the underlying waves $x(\xi, \varepsilon)$ and $x_{R}(\xi)$ are such that $x(\xi, \varepsilon) \rightarrow x_{R}(\xi)$ uniformly on compact $\xi$-intervals, it follows for each fixed $\xi \geq \xi_{R}$ that

$$
\lim _{\varepsilon \rightarrow 0} \hat{f}_{1}\left(\xi, \lambda_{n}, \varepsilon_{n}\right)=\hat{f}_{1 R}(\xi, \lambda) \text {. }
$$

We also have that

$$
\lim _{\xi \rightarrow+\infty} \hat{f}_{1 R}(\xi, \lambda)=\hat{e}_{1 R C}^{+}(\lambda)
$$

The behavior of the four curves $\hat{y}, \hat{y}_{R}, \hat{f}_{1}$, and $\hat{f}_{1 R}$ as functions of $\xi$ into $\mathbb{C} P^{3}$ are qualitatively depicted in Figure 5.1.

Let $\eta_{R}, d_{R}$ be the defining parameters for the right-hand slow tube $\Omega_{R}$ of $\widehat{\Omega}^{u}$ and let $\xi_{R}=\xi_{R}\left(d_{R}\right)$. For $\xi \geq \xi_{R}$ the coordinate system $\tilde{y}$ on $\mathbb{C} P^{3}$ is valid and the family of norms $|\cdot|_{\xi}$ is well defined. It follows from (5.22) that there exists $\xi_{1} \geq \xi_{R}$ such that

$$
\left|\tilde{f}_{1 R}(\xi, \lambda)-\tilde{e}_{1 R C}^{+}(\lambda)\right|_{\xi}<\eta_{R} / 4 \text { for all } \xi \geq \xi_{1} \text {. }
$$

Since we are assuming that alternative (1) is valid there exists $\xi_{2} \geq \xi_{R}$ such that

$$
\left|\tilde{y}_{R}(\xi, \lambda)-\tilde{e}_{1 R C}^{+}(\lambda)\right|_{\xi}<\eta_{R} / 4 \text { for all } \xi \geq \xi_{2} .
$$

Next, fix $\xi_{3}>\xi_{R}$; it follows from (5.21) for small $\varepsilon_{n}$ that

$$
\left|\tilde{f}_{1}\left(\xi, \lambda_{n}, \varepsilon_{n}\right)-\tilde{f}_{1 R}(\xi, \lambda)\right|_{\xi}<\eta_{R} / 4 \text { for } \xi_{R} \leq \xi \leq \xi_{3} .
$$




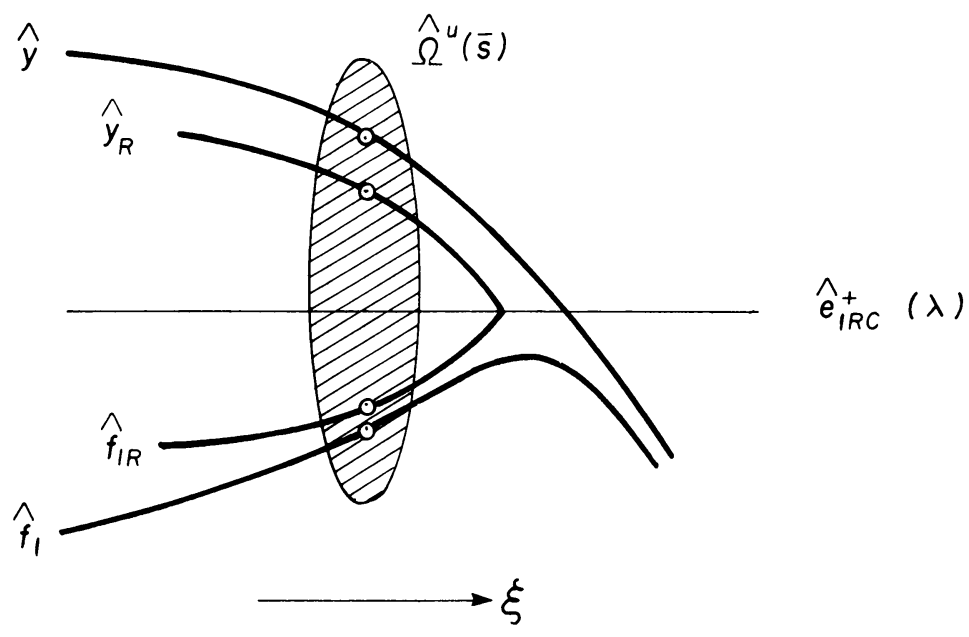

FIGURE 5.1

Finally, let $\xi_{4}>\max \left\{\bar{\xi}, \xi_{R}\right\}$ be given. We claim that

$$
\lim _{\varepsilon_{n} \rightarrow 0} \hat{y}\left(\xi, \lambda_{n}, \varepsilon_{n}\right)=\hat{y}_{R}(\xi, \lambda) \quad \text { uniformly on } \bar{\xi} \leq \xi \leq \xi_{4} \text {. }
$$

By definition of $\hat{y}_{R},(5.28)$ holds at $\xi=\bar{\xi}$. It then follows from estimate (5.7) and standard continuous dependence theorems for flows that (5.26) is valid on the compact interval $\bar{\xi} \leq \xi \leq \xi_{4}$. (More precisely, the interval can be partitioned so that on each subinterval a local coordinate system on $\mathbb{C} P^{3}$ can be employed; an inductive proof then follows from (5.7) and Gronwall's inequality.)

Now fix $\xi_{1}$ and $\xi_{2}$ in (5.23) and (5.24). Next, select $\xi_{3}$ in (5.25) and $\varepsilon_{4}$ in (5.26) so that

$$
\bar{s}=\max \left\{\xi_{1}, \xi_{2}\right\}<\min \left\{\xi_{3}, \xi_{4}\right\} .
$$

With $\xi_{4}$ fixed as above, it follows from (5.26) that

$$
\left|\tilde{y}\left(\bar{s}, \lambda_{n}, \varepsilon_{n}\right)-\hat{y}_{R}(\bar{s}, \lambda)\right|_{\bar{s}}<\eta_{R} / 4 .
$$

Combining the above with (5.23), (5.24), and (5.25) yields

$$
\left|\tilde{y}\left(\bar{s}, \lambda_{n}, \varepsilon_{n}\right)-\tilde{f}_{1}\left(\bar{s}, \lambda_{n}, \varepsilon_{n}\right)\right|_{\bar{s}}<\eta_{R}
$$

which implies that $\hat{y} \in \widehat{\Omega}^{u}$ at $\xi=\bar{s}$; this contradicts the postulated behavior of $\hat{y}$ at $\xi=+\infty$.

Corollary 5.8. Let $E_{2} \in \pi^{-1} \widehat{E}_{2}$ be a solution chosen so that (5.18) holds for each $\varepsilon>0$. Then $\left|E_{2}(\zeta, \lambda, \varepsilon)\right|$ is uniformly bounded for $|\zeta| \leq 1$ and for $0<\varepsilon \leq \bar{\varepsilon}$.

F. Proof of Theorem 5.3. We can now complete the proofs of (a), (b), and (c) of the theorem.

Proof of (a). Fix $a<A$ as in the statement of the theorem and suppose that $a \leq \zeta \leq A$. Pick a representative $E_{2}$ of $\pi^{-1} \widehat{E}_{2}$ so that $|Z| \leq 1$ on $a \leq \zeta \leq A$ 
for each $\varepsilon, 0<\varepsilon \leq \bar{\varepsilon}$. It follows that

$$
Z\left(1, \lambda, \varepsilon_{n}\right) \rightarrow Z^{+} \in \pi^{-1} \widehat{Z}^{+}
$$

as $\varepsilon_{n} \rightarrow 0$, where $\left|Z^{+}\right| \leq 1$. Let $Z_{*}(\zeta, \lambda)$ be the solution of $(5.4)_{R}$ in $\pi^{-1} \hat{Z}_{*}(\zeta, \lambda)$ with data $Z_{*}(1, \lambda)=Z^{+}$. We will show that $\left|Z-Z_{*}\right|$ tends uniformly to zero on the interval $a \leq \zeta \leq A$ as $\varepsilon \rightarrow 0$.

By Corollary 2.2 it follows that the coefficients $C$ and $H$ in (5.2b) and (5.4) converge uniformly to $C_{R}$ and $H_{R}$ as $\varepsilon \rightarrow 0$ for $a \leq \zeta \leq A$. It follows that the coefficients in (5.3) converge uniformly to those in $(5.3)_{R}$ on $a \leq \zeta \leq A$ and that the data of the former converge to those of the latter, as $\varepsilon_{n} \rightarrow 0$. The convergence of $Z$ to $Z_{*}$ will then follow from Gronwall's inequality if it can be shown that the forcing term $G \Gamma$, tends to zero uniformly on $a \leq \zeta \leq A$. This can be seen as follows. By Corollary 5.6,

$$
\widehat{E}_{2}(\zeta, \lambda, \varepsilon) \in N_{\delta}\left(\widehat{\Sigma}_{s}(\zeta, \lambda, \varepsilon)\right)
$$

for $a \leq \zeta$ and all small $\varepsilon$, where $\delta>0$ is arbitrary. The slow subspace $\Sigma_{s}(\zeta, \lambda, \varepsilon)$ can be characterized for small $\varepsilon$ by computing the limits of its basis, $F_{i}(\zeta, \lambda, \varepsilon), i=2,3$, as $\varepsilon \rightarrow 0$. By an analysis similar to that used in the derivation of $(3.7 b)$ it can be seen that these limits are

$$
\begin{aligned}
& F_{2} \rightarrow F_{2 R}(\zeta, \lambda)=\left(H_{R}(\zeta, \lambda), 0,1, m_{p}(\zeta, \lambda)\right)^{t}, \\
& F_{3} \rightarrow F_{3 R}(\zeta, \lambda)=\left(H_{R}(\zeta, \lambda), 0,1, m_{n}(\zeta, \lambda)\right)^{t}
\end{aligned}
$$

for suitable coefficients $m_{p}, m_{n}$ as in (3.7c). From the above and our choice of the representative $E_{2}$ in $\pi^{-1} E_{2}$ it follows that

$$
E_{2} \rightarrow \alpha_{2} F_{2 R}+\alpha_{3} F_{3 R} \quad \text { at }(\zeta, \lambda)
$$

for some coefficients $\alpha_{i}(\zeta, \lambda), i=2,3$, which remain uniformly bounded on $a \leq \zeta \leq A$. The above convergence is uniform on this interval. Also, we have that $\Gamma$ can be expressed as

$$
\Gamma=P_{1}-H_{R}(\zeta, \lambda) P_{2}+\left(H(\zeta, \lambda, \varepsilon)-H_{R}(\zeta, \lambda)\right) P_{2}
$$

since $H$ converges uniformly to $H_{R}$ and $\left|P_{2}\right| \leq 1$ on $a \leq \zeta \leq A$ we see from (5.27) and (5.28) that $|\Gamma|$ tends uniformly to zero on $a \leq \zeta \leq A$. This establishes the uniform convergence of $Z$ to $Z_{*}$ on $a \leq \zeta \leq A$.

It also follows immediately from (5.27), (5.28) and Corollary 5.6 that the 1-components of $E_{2}$ converge uniformly to those of $E_{*}$ on this interval.

The convergence of $E_{2}$ to $E_{*}$ on $-A \leq \zeta \leq-a$ is proved in a similar manner.

Proof of $(\mathrm{b})$. Let $Z^{ \pm}(\zeta, \lambda)$ be a representative of $\pi^{-1} \hat{Z}^{ \pm}(\zeta, \lambda)$; recall that $\widehat{Z}^{ \pm}(\zeta, \lambda)$ are the solutions of $(5.5)_{R}$ comprising the portions of $\hat{Z}_{*}(\zeta, \lambda)$ in $\zeta>0$ and $\zeta<0$. We may assume that $E_{2} \in \pi^{-1} \widehat{E}_{2}$ is chosen so that $(5.18)$ holds; by (a) we may therefore select $Z^{ \pm}$so that $\left|Z^{ \pm}\right| \leq 1$ on $0<|\zeta| \leq$ 
1 . Since $Z^{ \pm}(\zeta, \lambda)$ satisfy the equations $(5.3)_{R}$, whose right-hand sides are uniformly bounded, the one-sided limits

$$
B^{ \pm}(\lambda)=\lim _{\zeta \rightarrow 0^{ \pm}} Z^{ \pm}(\zeta, \lambda)
$$

both exist. We will show that $B^{-}(\lambda)=B^{+}(\lambda)$.

Let $\delta>0$ be given; select $a_{1}>0$ so small that

$$
\left|Z^{ \pm}\left( \pm a_{1}, \lambda\right)-B^{ \pm}(\lambda)\right|<\delta / 5 .
$$

Next, note that every term on the right-hand side of the equations (5.3) for the 2-components of $E_{2}$ is uniformly bounded on the interval $|\zeta| \leq 1$ for $0<\varepsilon \leq \bar{\varepsilon}$. This follows from the choice of scaling (5.18) for $E_{2}$ and from Corollary 5.8. Thus $\dot{Z}(\zeta, \lambda, \varepsilon)$ is uniformly bounded for such $\zeta$ and $\varepsilon$ and so there exists $a \in\left(0, a_{1}\right)$ such that

$$
|Z(a, \lambda, \varepsilon)-Z(-a, \lambda, \varepsilon)|<\delta / 5
$$

for $0<\varepsilon \leq \bar{\varepsilon}$. Finally, fix $a$ as above; it follows from part (a) of this theorem that

$$
\left|Z(a, \lambda, \varepsilon)-Z^{+}(a, \lambda)\right|<\delta / 5, \quad\left|Z(-a, \lambda, \varepsilon)-Z^{-}(-a, \lambda)\right|<\delta / 5,
$$

for $0<\varepsilon \leq \bar{\varepsilon}$. Combining all of the above yields $\left|B^{-}(\lambda)-B^{+}(\lambda)\right|<\delta$, as required.

Proof of (c). Let $\widehat{E}_{*}$ and $\widehat{E}_{2 R}$ be the solutions defined above the statement of Theorem 5.3. The defining condition for $\widehat{E}_{2 R}$ at $\zeta=-\infty$ together with the assumption that $\lambda$ is not an eigenvalue of $(3.9)_{s R}$ imply that $\widehat{E}_{2 R}(\zeta, \lambda)$ tends to $\hat{e}_{2 R}^{ \pm}(\lambda)$ at $\zeta= \pm \infty$. The difficulty is that the asymptotic behavior of $\hat{E}_{*}$, a solution of $(3.9)_{s R}$ which is obtained as a limit of $\widehat{E}_{2}(\zeta, \lambda, \varepsilon)$ for finite $\zeta$ as $\varepsilon \rightarrow 0$, is not known at this point. More precisely, although the limits

$$
\begin{array}{cc}
\lim _{\zeta \rightarrow-\infty} \widehat{E}_{2}(\zeta, \lambda, \varepsilon)=\hat{e}_{2}^{-}(\lambda, \varepsilon), & \varepsilon>0, \\
\lim _{\varepsilon \rightarrow 0} \widehat{E}_{2}(\zeta, \lambda, \varepsilon)=\widehat{E}_{*}(\zeta, \lambda), \quad \zeta \neq 0,
\end{array}
$$

are known, it has not yet been proved that the second limit is uniform in $\zeta$ for $|\zeta| \geq a>0$, and so, the possibility arises that $\widehat{E}_{2}$ and $\widehat{E}_{*}$ could have substantially different asymptotic behavior as $\zeta \rightarrow \pm \infty$. The key to characterizing that of $\widehat{E}_{2}$ is to first determine the behavior of $\widehat{E}_{*}$ for large $|\zeta|$. In fact, we will show that $\widehat{E}_{*}$ coincides identically with $\widehat{E}_{2 R}$ by showing that $\widehat{E}_{*}$ tends to $\hat{e}_{2 R}^{-}(\lambda)$ at $\zeta=-\infty$. (Recall that this condition uniquely defines $\hat{E}_{2 R}$.) Once this has been established, a similar argument will imply that $\widehat{E}_{*}$ and $\widehat{E}_{2}$ have nearly the same behavior near $\zeta=\infty$, which will complete the proof.

It will be convenient at this point to introduce a new local coordinate system on $\mathbb{C} P^{3}$. Let $\mathbb{C} P_{P_{2}}^{2}$ denote the copy of $\mathbb{C} P^{2}$ inside $\mathbb{C} P^{3}$ along which $P_{2}=0$ 
in the fibers and, given $\alpha>0$, let

$$
\mathbb{C} P_{\alpha}^{3}=\left\{\widehat{Y} \in \mathbb{C} P^{3}: \rho\left(\widehat{Y}, \mathbb{C} P_{P_{2}}^{2}\right) \geq \alpha\right\},
$$

where $\rho$ is the metric on $\mathbb{C} P^{3}$ introduced earlier. For $\hat{Y}$ in $\mathbb{C} P_{\alpha}^{3}$ we may use local coordinates

$$
B_{1}=P_{1} / P_{2}, \quad B_{2}=Q_{1} / P_{2}, \quad S=Q_{2} / P_{2}
$$

the slow projectivized equations can then be expressed in $\left(B_{1}, B_{2}, S\right)$ as

$$
\begin{aligned}
& \varepsilon \dot{B}_{1}=B_{2}-\varepsilon B_{1} S \\
& \varepsilon \dot{B}_{2}=-\theta B_{2}-\left[A_{11}(\zeta, \varepsilon) B_{1}-A_{12}(\zeta, \varepsilon)\right]-\varepsilon B_{2} S, \\
& \dot{S}=C(\zeta, \lambda, \varepsilon)-S^{2}-\varepsilon \theta S-A_{21}(\zeta, \varepsilon)\left(B_{1}-H(\zeta, \lambda, \varepsilon)\right),
\end{aligned}
$$

where $H$ and $C$ are as in (5.2) and (5.4). In the following, $\left(B_{1}, B_{2}, S\right)$ will denote the solution of (5.31) associated with $\widehat{E}_{2}$, which is obtained by forming the quotients (5.30) from any representative $E_{2} \in \pi^{-1} \widehat{E}_{2}$.

Proposition 5.9. Suppose that $\widehat{E}_{2}(\zeta, \lambda, \varepsilon) \in \mathbb{C} P_{\alpha}^{3}$ for $a \leq|\zeta| \leq A$, where $A \leq$ $\infty$, for $0<\varepsilon \leq \bar{\varepsilon}$ and for some $\alpha>0$. Given $\delta>0$ there exists $\bar{\varepsilon}=\bar{\varepsilon}(\delta)$ such that

$$
\left|B_{2}\right| \leq \delta, \quad\left|B_{1}-H(\zeta, \lambda, \varepsilon)\right| \leq \delta
$$

on $a \leq|\zeta| \leq A$ for $\varepsilon \leq \bar{\varepsilon}(\delta)$.

Proof. The proposition simply states that $\widehat{E}_{2}$ remains near the slow subspace $\widehat{\Sigma}_{s}$ in terms of the local coordinates $\left(B_{1}, B_{2}, S\right)$. This is a consequence of Corollary 5.6. It can also be seen directly from the equations (5.31) by noting that the hypothesis $\widehat{E}_{2} \in \mathbb{C} P_{\alpha}^{3}$ is equivalent to a uniform bound

$$
\left|B_{1}\right|,\left|B_{2}\right|,|S| \leq L, \quad a \leq|\zeta| \leq A,
$$

where $L$ depends only on $\alpha$ and the metric $\rho$ on $\mathbb{C} P^{3}$ since without loss of generality we can take $P_{2}=1$ in (5.30). The proof then proceeds by assuming that either

$$
\left|B_{2}\right| \geq \delta \quad \text { or }\left|B_{1}-H(\zeta, \lambda, \varepsilon)\right| \geq \delta
$$

for some sequence $\varepsilon_{n} \rightarrow 0$; it is then easily seen from the first two equations in (5.31) that the postulated uniform bounds on $\left|B_{1}\right|$ and $\left|B_{2}\right|$ would then be violated in slow $(\zeta)$ time of order $L \varepsilon / \delta$.

We can now complete the proof of the theorem. The solution $\widehat{E}_{*}$ is determined by the equation $(5.5)_{R}$ for $\widehat{Z}_{*}$. This equation can be expressed in the local coordinate $S_{*}=Q_{2 *} / P_{2 *}$ as

$$
\dot{S}_{*}=C_{R}(\zeta, \lambda)-S_{*}^{2}
$$

Noting that

$$
\pm\left[C_{R}(-\infty, \lambda)\right]^{1 / 2}=m_{n}^{-}(\lambda), m_{p}^{-}(\lambda), \quad \pm\left[C_{R}(+\infty, \lambda)\right]^{1 / 2}=m_{n}^{+}(\lambda), m_{p}^{+}(\lambda),
$$


where the latter quantities are as in (3.7c), it follows that (5.32) is hyperbolic at $\zeta= \pm \infty$ with saddle points at each end. It follows that the only possible behavior for $\widehat{E}_{*}(\zeta, \lambda)$ as $\zeta \rightarrow-\infty$ is one of the following

(a) $\hat{E}_{*}(\zeta, \lambda) \rightarrow \hat{e}_{2 R}^{-}(\lambda)$,

(b) $\widehat{E}_{*}(\zeta, \lambda) \rightarrow \hat{e}_{3 R}^{-}(\lambda)$.

We contend that the convergence is as in case (a). Assume to the contrary that case (b) occurs. This is equivalent to supposing that $S_{*}(\zeta, \lambda)$ tends to $m_{n}^{-}(\lambda)$ as $\zeta \rightarrow-\infty$.

The asymptotic system associated with (5.32) at $\zeta=-\infty$ is

$$
\dot{S}=C_{R}(-\infty, \lambda)-S^{2}=-\left(m_{n}^{-}(\lambda)-S\right)\left(m_{p}^{-}(\lambda)-S\right) .
$$

Since $\operatorname{Re} m_{n}^{-}(\lambda)<0$ and $\operatorname{Re} m_{p}^{-}(\lambda)>0$, the former critical point is a repeller and the latter critical point is an attractor. Let $\eta>0$ be given, and let $N \subset \mathbb{C}$ be a repelling neighborhood of $m_{n}^{-}(\lambda)$ for (5.33) of radius $\eta>0$. We will show that there exists $\zeta_{*}<0$ such that $S(\zeta, \lambda, \varepsilon) \in N$ for all sufficiently small $\varepsilon>0$ and for all $\zeta \leq \zeta_{*}$.

By hypothesis, there exists $\zeta_{1}<0$ such that $S_{*}(\zeta, \lambda) \in N$ for all $\zeta \leq \zeta_{1}$; here $\zeta_{1}$ depends only on $\eta$. By $(5.29 \mathrm{~b})$ it follows for each fixed $\zeta \leq \zeta_{1}$ that

$$
S(\zeta, \lambda, \varepsilon) \in N
$$

for all sufficiently small $\varepsilon$, where $\left(B_{1}, B_{2}, S\right)$ are the local coordinates (5.30) for $\widehat{E}_{2}$.

We claim that there exists $\alpha>0$ depending only on $\eta$ such that $\widehat{E}_{2} \in \mathbb{C} P_{\alpha}^{3}$ whenever $S(\zeta, \lambda, \varepsilon) \in N$ and $\zeta \leq \zeta_{1}$. To this end, note that by $(3.7 \mathrm{~b}, \mathrm{c})$ and by Corollary 2.2 we can express the spanning vectors $F_{2}$ and $F_{3}$ for $\Sigma_{s}(\zeta, \lambda, \varepsilon)$ as

$$
\begin{aligned}
& F_{2}(\zeta, \lambda, \varepsilon)=\left(C_{R}(\zeta, \lambda), 0,1, m_{p}(\zeta, \lambda)\right)^{t}+\mathscr{O}(\delta), \\
& F_{3}(\zeta, \lambda, \varepsilon)=\left(C_{R}(\zeta, \lambda), 0,1, m_{n}(\zeta, \lambda)\right)^{t}+\mathscr{O}(\delta),
\end{aligned}
$$

where $\delta>0$ is arbitrary and $\varepsilon$ is sufficiently small relative to $\delta$; from $(3.7 \mathrm{~b})$ we have that $m_{n}(\zeta, \lambda)=-m_{p}(\zeta, \lambda)$. Assuming that $\widehat{E}_{2} \in \mathbb{C} P_{\alpha}^{3}$ for some $\alpha>0$ it follows that $E_{2} \in \pi^{-1} \widehat{E}_{2}$ can be chosen so that

$$
E_{2}(\zeta, \lambda, \varepsilon)=\left(B_{1}, B_{2}, 1, S\right) \text { at }(\zeta, \lambda, \varepsilon) \text {. }
$$

By Corollary 5.6, there exist $a, b \in \mathbb{C}$ such that $E_{2}=a F_{2}+b F_{3}+\mathscr{O}(\delta)$, so that $a+b=1$. From the above it then follows that at $(\zeta, \lambda, \varepsilon)$

$$
E_{2}\left(C_{R}(\zeta, \lambda), 0,1,(a-b) m_{n}(\zeta, \lambda)\right)^{t}+\mathscr{O}(\delta)
$$

for sufficiently small $\varepsilon$. It follows that $\left|B_{1}\right|,\left|B_{2}\right|$, and $|S|$ will be uniformly bounded while $S$ remains in $N$, so that $\widehat{E}_{2}$ will satisfy the estimates in Proposition 5.9 for all sufficiently small $\varepsilon$ and for all $\zeta \leq \zeta_{1}$ for which $S$ remains in $N$. 
The third equation in (5.31) for $S$ can be expressed as

$$
\begin{aligned}
\dot{S}= & C_{R}(-\infty, \lambda)-S^{2}+d(\zeta, \lambda, \varepsilon), \\
d(\zeta, \lambda, \varepsilon)= & C(\zeta, \lambda, \varepsilon)-C_{R}(-\infty, \lambda)-\varepsilon \theta S \\
& -A_{21}(\zeta, \varepsilon)\left(B_{1}-H(\zeta, \lambda, \varepsilon)\right) .
\end{aligned}
$$

This is a perturbation of the asymptotic equation (5.33). There exists $\bar{d}>0$ depending on $\eta$ such that if $|d(\zeta, \lambda, \varepsilon)| \leq \bar{d}$, then $N$ is a repelling neighborhood of $m_{n}^{-}(\lambda)$ for equations (5.35). By Corollary 2.2 and Proposition 5.9 there exists $\zeta_{*} \leq \zeta_{1}$ such that $|d(\zeta, \lambda, \varepsilon)| \leq \bar{d}$ for $\zeta \leq \zeta_{*}$ and sufficiently small $\varepsilon$, provided that $S(\zeta, \lambda, \varepsilon) \in N$. If we now choose $\varepsilon$ so small that (5.34) holds at $\zeta=\zeta_{*}$ the above remarks show that the set $N$ is negatively invariant for (5.35) for all $\zeta \leq \zeta_{*}$. It then follows that $\widehat{E}_{2}(\zeta, \lambda, \varepsilon)$ remains in a small neighborhood of $\hat{e}_{3 R}^{-}(\lambda)$ as $\zeta \rightarrow-\infty$, contradicting (a) of Theorem 5.1.

We have now proved that $\widehat{E}_{*}$ converges to $\hat{e}_{2 R}^{-}(\lambda)$ as $\zeta \rightarrow-\infty$. This condition uniquely determines $\widehat{E}_{*}$, and so $\widehat{E}_{*}$ and $\widehat{E}_{2 R}$ coincide identically for $\zeta<0$. Furthermore, by part (b) of Theorem 5.3, $\widehat{E}_{*}$ and $\widehat{E}_{2 R}$ satisfy the same matching condition across $\zeta=0$ and so they coincide for all $\zeta>0$. By the hypothesis in (c) of Theorem 5.3, $\widehat{E}_{2 R}$ and hence, $\widehat{E}_{*}$, tends to $\hat{e}_{2 R}^{+}(\lambda)$ as $\zeta \rightarrow+\infty$. The proof of the theorem is completed by constructing a positively invariant set $N$ for (5.35) about the attracting critical point $m_{p}^{+}(\lambda)$ for the associated asymptotic system at $\zeta=+\infty$. This, together with Proposition 5.9, forces $\hat{E}_{2}$ to remain in a neighborhood of $\hat{e}_{2}^{+}(\lambda, \varepsilon)$ near $\zeta=+\infty$. The details of this part of the proof are the same as the argument given for $\zeta$ near $-\infty$ and they will therefore be omitted.

\section{COMPUTATION OF THE CHERN NUMBER}

A. The Whitney sum decomposition. We now turn to the bundle $\mathscr{E}=\mathscr{E}(\varepsilon)$ over $S^{2}$ whose construction was sketched in §IIIE. The crucial point in forming this bundle is that $\lambda \in K$ must not be an eigenvalue of $(3.3)_{f}$. Corollary 4.5 and (c) of Theorem 5.3 together imply the following result.

Theorem 6.1. Suppose that $\lambda \in K$ is not an eigenvalue of either (3.9) ${ }_{f R}$ or of $(3.9)_{s R}$. Then there exists $\bar{\varepsilon}>0$ such that $\lambda$ is not an eigenvalue of $(3.3)_{f}$ for $0<\varepsilon \leq \bar{\varepsilon}$.

It has previously been described how $K$ may be chosen so that the first condition is satisfied (see $\S I V D$ ). Later (see $\S$ VIE) we will show that a similar choice of $K$ will also suffice in satisyfing the second condition.

Corollary 4.5 and Theorem 5.3 yield further insight into the structure of the 2-plane bundle $\mathscr{E}(\varepsilon)$. In particular, these results allow us to continuously extend $\hat{e}_{1}$ and $\hat{e}_{2}$ to $[-1,1] \times K$ so that the bundles can be capped at $\tau=-1$ and $\tau=+1$ in a manner analogous to that of $\mathscr{E}(\varepsilon)$ (see $\S I I I F)$. This procedure yields a pair of line bundles $\mathscr{E}_{1}(\varepsilon)$ and $\mathscr{E}_{2}(\varepsilon)$ over $S^{2}$. It then follows from a 
general construction that

$$
\mathscr{E}(\varepsilon)=\mathscr{E}_{1}(\varepsilon) \oplus \mathscr{E}_{2}(\varepsilon)
$$

and that

$$
c_{1}(\mathscr{E}(\varepsilon))=c_{1}\left(\mathscr{E}_{1}(\varepsilon)\right)+c_{1}\left(\mathscr{E}_{2}(\varepsilon)\right)
$$

for sufficiently small $\varepsilon>0$ (see remark following the main theorem in [AGJ]). The computation of $c_{1}$ of $\mathscr{E}_{1}(\varepsilon)$ and $\mathscr{E}_{2}(\varepsilon)$ proceeds as follows. We will define certain reduced line bundles $\mathscr{E}_{1 R}$ and $\mathscr{E}_{2 R}$ directly from the reduced equations $(3.9)_{f R}$ and $(3.9)_{s R}$. The geometric information contained in the estimates of $\S \S \mathrm{IV}$ and $\mathrm{V}$ will then enable us to construct equivalences between $\mathscr{E}_{i}(\varepsilon)$ and $\mathscr{E}_{i R}, i=1,2$. Finally, the spectrum of each reduced eigenvalue problem is characterized, and the Chern number theorem of $[A G J]$ is used "in reverse" to compute the $c_{1}$ of the reduced bundles.

By Lemma 3.3, the proof of the Stability Theorem will be complete if it can be shown that $c_{1}(\mathscr{E}(\varepsilon))=1$. This is proved in Lemma 6.6 in subsection $\mathrm{E}$.

B. The reduced bundles. The fast reduced bundle, $\mathscr{E}_{1 R}$, and the slow reduced bundle, $\mathscr{E}_{2 R}$, are defined as follows.

Definition of $\mathscr{E}_{1 R}$. Since $\lambda \in K$ is not an eigenvalue of $(3.9)_{f R}$, for each such $\lambda$ there exists a solution $\hat{e}_{1 R}(\xi, \lambda)$ of the reduced projectivized equations (4.22) which connects the fast unstable direction $\hat{e}_{1 R C}^{-}(\lambda)$ in the left-hand corner at $\xi=-\infty$ to the fast unstable direction $\hat{e}_{1 R C}^{+}(\lambda)$ in the right-hand corner at $\xi=+\infty$. The variable $\xi$ is compactified through a reparametrization $\xi \rightarrow \tau$ where $-1 \leq \tau \leq 1$, with $\tau( \pm \infty)= \pm 1$. In particular, (4.22) is augmented with

$$
\tau^{\prime}=\kappa\left(1-\tau^{2}\right), \quad \tau(0)=0
$$

where $\kappa>0$. We may therefore regard $\hat{e}_{1 R}(\xi, \lambda)$ as a function of $(\tau, \lambda)$.

The base space for $\mathscr{E}_{1 R}$ is

$$
B=\{-1\} \times K^{0} \cup(-1,1) \times K \cup\{1\} \times K^{0}
$$

(recall that $K^{0}$ is the region interior to $K$ ). Our solution $\hat{e}_{1 R}(\xi, \lambda)$ is chosen so that

$$
\hat{e}_{1 R}(\tau, \lambda) \rightarrow \hat{e}_{1 R C}^{ \pm}(\lambda) \quad \text { as } \tau \rightarrow \pm 1, \lambda \in K,
$$

where $\hat{e}_{1 R C}^{ \pm}(\lambda)$ is the image of the fast unstable direction in the left- and righthand corners, and since $e_{1 R C}^{ \pm}(\lambda)$ are both well defined for $\lambda \in K^{0} \cup K$ we can continuously extend $\hat{e}_{1 R}$ to all of $B$ by defining

$$
\hat{e}_{1 R}( \pm 1, \lambda)=\hat{e}_{1 R C}^{ \pm}(\lambda) \quad\left(\lambda \in K \cup K^{0}\right) .
$$

The bundle $\mathscr{E}_{1 R}$ is defined as the pullback of the universal bundle induced by the map $\hat{e}_{1 R}: B \rightarrow \mathbb{C} P^{3}$ :

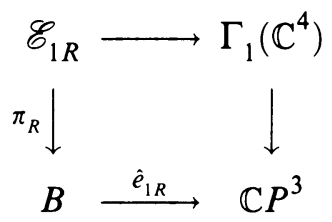


(see §IIIF), i.e., the fiber over $b \in B$ is the complex line in $\mathbb{C}^{4}$ associated with $\hat{e}_{1 R}(b)$.

We remark that $p_{2} \equiv q_{2} \equiv 0$ in $\pi^{-1} \hat{e}_{1 R}$, hence the ambient space can be regarded as $B \times \mathbb{C}^{2}$ instead of $B \times \mathbb{C}^{4}$.

Definition of $\mathscr{E}_{2 R}$. Let $\widehat{E}_{2 R}(\zeta, \lambda)$ be the projectivization of the solution $E_{2 R}(\zeta, \lambda)$ defined above the statement of Theorem 5.3. We reparametrize $\widehat{E}_{2 R}$ by changing $\zeta$ to $T$, where $T=T(\zeta)$ satisfies

$$
\dot{T}=\kappa\left(1-T^{2}\right), \quad T(0)=0
$$

and $-1 \leq T \leq 1$. Express the reparametrized curve as $\widehat{E}_{2 R}(T, \lambda)$. The base space $B$ is the same as in (6.3) with $\tau$ replaced by $T$

For $\zeta \in K, \widehat{E}_{2 R}(T, \lambda)$ tends to $\hat{e}_{2 R}^{ \pm}(\lambda)$ as $T$ tends to \pm 1 since $\lambda$ is not an eigenvalue of $(3.9)_{s R}$. The map $\widehat{E}_{2 R}$ can therefore be continuously extended to the caps by defining

$$
\widehat{E}_{2 R}( \pm 1, \lambda)=\hat{e}_{2 R}^{ \pm}(\lambda), \quad\left(\lambda \in K \cup K^{0}\right) .
$$

Our map is not well defined at $T=0$ since the original solution $E_{2 R}$ is discontinuous at $\zeta=0$. In order to obtain a continuous bundle, consider the left and right halves

$$
B_{-}=B \cap\{T \leq 0\}, \quad B_{+}=B \cap\{T \geq 0\} .
$$

Bundles $\mathscr{E}_{2 R}^{-}$and $\mathscr{E}_{2 R}^{+}$can be defined over each hemisphere $B_{-}$and $B_{+}$from the pullback of $\widehat{E}_{2 R}$ restricted to $B_{-}$and to $B_{+}$. A bundle $\mathscr{E}_{2 R}$ over the whole sphere is constructed by gluing the fibers over $B_{-} \cap B_{+}=\{0\} \times K$ together in a suitable manner.

In order to specify the gluing map we first recall that $\widehat{E}_{2 R}$ was constructed from a solution $\hat{Z}_{2 R}(\zeta, \lambda)$ of the projectivized, reduced equations for the 2components. $\widehat{Z}_{2 R}$ is continuous across $\zeta=0$ and decays to the unstable eigenvectors of $B_{R}( \pm \infty, \lambda)$ as $\zeta \rightarrow \pm \infty$. For $Z(\zeta, \lambda) \in \pi^{-1} \hat{Z}_{2 R}(\zeta, \lambda), E_{2 R}(\zeta, \lambda)$ was defined by the map $i_{R}(\zeta, \lambda): \mathbb{C}^{2} \rightarrow \mathbb{C}^{4}$, namely

$$
E_{2 R}(\zeta, \lambda)=i_{R}(\zeta, \lambda) Z(\zeta, \lambda)
$$

Let

$$
i_{R}^{ \pm}(\lambda)=\lim _{\zeta \rightarrow 0^{ \pm}} i_{R}(\zeta, \lambda)
$$

The fibers of $\mathscr{E}_{2 R}^{ \pm}$over $B^{+} \cap B^{-}$can then be expressed as $\mathscr{E}_{2 R}^{ \pm}=\operatorname{span}\left\{i_{R}^{ \pm}(\lambda) Z\right\}$, where $Z \in \pi^{-1} \widehat{Z}_{R}(0, \lambda)$ and $\pi: \mathbb{C}^{2} \rightarrow \mathbb{C} P^{1}$ is the projection map. By fixing a choice of $Z$ we obtain an isomorphism

$$
\varphi_{R}: \mathscr{E}_{2 R}^{-}\left|\{0\} \times K \rightarrow \mathscr{E}_{2 R}^{+}\right|\{0\} \times K,
$$

where $\mathscr{E} \mid A$ denotes the restriction of a bundle $\mathscr{E}$ to a subset $A$ of its base space. In particular, define

$$
\varphi_{R}(\lambda) i_{R}^{-}(\lambda) Z=i_{R}^{+}(\lambda) Z
$$


and extend $\varphi_{R}(\lambda)$ linearly. The bundle $\mathscr{E}_{2 R}$ is defined by the "clutching" operation,

$$
\mathscr{E}_{2 R}=\mathscr{E}_{2 R}^{-} \cup_{\varphi_{R}} \mathscr{E}_{2 R}^{+}
$$

(see Atiyah [A]). This is the bundle obtained by gluing $\mathscr{E}_{2 R}^{ \pm}$together at $\{0\} \times K$ via the equivalence relation

$$
y_{-} \sim \varphi_{R}(\lambda) y_{-},
$$

where $y_{-} \in \pi^{-1} \mathscr{E}_{2 R}^{-} \mid(0, \lambda)$.

The following facts are proved in $[\mathrm{A}]$.

Lemma 6.2. $\mathscr{E}_{2 R}$ is a bundle over $B$ (i.e., it satisfies the local triviality condition at $\{0\} \times K)$, and it is uniquely determined modulo bundle equivalences by the homotopy type of the gluing map $\varphi_{R}$.

We remark that $\mathscr{E}_{2 R}$ can be deformed to an equivalent bundle $\widetilde{\mathscr{E}}_{2 R}$ obtained from $\mathscr{E}_{2 R}$ by projecting the fibers onto their 2-components. In particular, for $Z \in \pi^{-1} \widehat{Z}_{R}(\zeta, \lambda)$, let $\mathscr{E}_{2 R}(s)$ be the bundle formed analogously to $\mathscr{E}_{2 R}$ via the maps defined by

$$
\begin{gathered}
i_{R}(\zeta, \lambda, s) Z=\left(s C_{R}(\zeta, \lambda) P_{2}, 0, P_{2}, Q_{2}\right)^{t}, \\
i_{R}^{ \pm}(\lambda, s)=\lim _{\zeta \rightarrow 0^{ \pm}} i_{R}(\zeta, \lambda, s), \\
\varphi_{R}(\lambda, s)\left[i_{R}^{-}(\lambda, s) Z\right]=i_{R}^{+}(\lambda, s) Z .
\end{gathered}
$$

Clearly $\mathscr{E}_{2 R}(0)=\tilde{\mathscr{E}}_{2 R}$ and $\mathscr{E}_{2 R}(1)=\mathscr{E}_{2 R} ;$ it follows from Lemma 6.2 that $\mathscr{E}_{2 R}$ and $\widetilde{\mathscr{E}}_{2 R}$ are equivalent bundles.

C. Continuation of $\mathscr{E}_{1}(\varepsilon)$ to $\mathscr{E}_{1 R}$. In this section we shall prove that $\mathscr{E}_{1}(\varepsilon)$ and $\mathscr{E}_{1 R}$ are equivalent bundles. It will first be convenient to reparametrize $\mathscr{E}_{1}(\varepsilon)$ so that its $\tau$-scaling agrees with that of $\mathscr{E}_{1 R}$. To this end, we replace the $\tau$-equation for $\mathscr{E}_{1}(\varepsilon)$,

$$
\tau^{\prime}=\varepsilon \kappa\left(1-\tau^{2}\right),
$$

by equation (6.2). This reparametrization of $\tau$ stretches the fibers over the transition to a layer of $\mathscr{O}(1)$, while it compresses the fibers over the slow manifolds into intervals of $\mathscr{O}(\varepsilon)$ at $\tau=-1$ and $\tau=+1$. This clearly does not change the topology of $\mathscr{E}_{1}(\varepsilon)$; more precisely, the rescaling induces a homeomorphism $h$ of the base space $B$ of $\mathscr{E}_{1}(\varepsilon)$; the new bundle obtained by the pullback operation $h^{*} \mathscr{E}_{1}(\varepsilon)$ is equivalent to $\mathscr{E}_{1}(\varepsilon)$. In the following we shall regard the $\tau$-scaling as given by (6.2) and continue to denote the associated bundle by $\mathscr{E}_{1}(\varepsilon)$.

The next lemma shows that $\mathscr{E}_{1}(\varepsilon)$ and $\mathscr{E}_{1 R}$ are close over the interior of $[-1,1] \times K$; however, they still differ significantly near the ends. 
Lemma 6.3. Let $\hat{e}_{1}(\tau, \lambda, \varepsilon)$ and $\hat{e}_{1 R}(\tau, \lambda)$ both have $\tau$ parametrized by (6.2). For each subinterval $\left[\tau_{1}, \tau_{2}\right] \subset(-1,1)$ we have that

$$
\lim _{\varepsilon \rightarrow 0} \hat{e}_{1}(\tau, \lambda, \varepsilon)=\hat{e}_{1 R}(\tau, \lambda)
$$

uniformly for $(\tau, \lambda) \in\left[\tau_{1}, \tau_{2}\right] \times K$.

Proof. The condition requiring that $\tau \in\left[\tau_{1}, \tau_{2}\right]$ is equivalent to supposing that $\xi$ lies in a compact interval $\left[\xi_{1}, \xi_{2}\right]$. In the following, it will be convenient to replace $\tau$ with $\xi$.

If the lemma were false, there would exist $\lambda \in K, \xi_{*} \in\left[\xi_{1}, \xi_{2}\right], \hat{e}_{*} \in \mathbb{C} P^{3}$, and $\varepsilon_{n} \rightarrow 0$ such that

$$
\lim _{\varepsilon_{n} \rightarrow 0} \hat{e}_{1}\left(\xi_{*}, \lambda, \varepsilon_{n}\right)=\hat{e}_{*} \neq \hat{e}_{1 R}\left(\xi_{*}, \lambda\right)
$$

Let $X_{1}$ be the copy of $\mathbb{C} P^{1}$ inside $\mathbb{C} P^{3}$ for which $p_{2}=q_{2}=0$ in the fibers. We claim that $\hat{e}_{*} \in X_{1}$. Let $e_{*} \in \pi^{-1} \hat{e}_{*}$ and suppose to the contrary that at least one of $p_{2^{*}}, q_{2^{*}}$ is nonzero. Let $e_{*}(\xi)$ be the solution of $(3.9)_{f R}$ satisfying $e_{*}\left(\xi_{*}\right)=e_{*}$; note that $p_{2^{*}}$ and $q_{2^{*}}$ are constant along $e_{*}(\xi)$.

We claim that $p_{1 *}(\xi)$ and $q_{1 *}(\xi)$ remain uniformly bounded for all $\xi \leq \xi_{*}$. If this were not the case it is easily seen by examining the equation for $q_{1 *} / p_{1 *}$ that $\hat{e}_{*}(\xi)$ would then tend to the fast stable direction in the left corner $\hat{e}_{4 R C}^{-}(\lambda)$. Hence there would exist $\bar{\xi} \leq \xi_{L}$ such that $\hat{e}_{*}(\bar{\xi}) \in \widehat{\Omega}^{s}(\bar{\xi})$; recall that $\widehat{\Omega}^{s}$ is a negatively invariant set containing $\hat{e}_{4}$. However by (6.5) and Gronwall's inequality it would then follow that $\hat{e}_{1} \in \widehat{\Omega}^{s}(\bar{\xi})$ at $\xi=\bar{\xi}$, contradicting the defining condition of $\hat{e}_{1}$ at $-\infty$.

Thus $p_{1 *}(\xi)$ and $q_{1 *}(\xi)$ remain uniformly bounded for all $\xi \leq \xi^{*}$. It follows that $\hat{e}_{*}(\xi)$ remains uniformly bounded away from $X_{1}$ for $\xi \leq \xi_{*}$, say

$$
\rho\left(\hat{e}_{*}(\xi), X_{1}\right) \geq \delta
$$

for some $\delta>0$ and $\xi \leq \xi_{*}$. From the form (3.7b) of the fast reduced unstable eigenvector it follows that there exist $\eta_{L}$ and $d_{L}$ depending on $\delta$ such that

$$
\rho\left(\widehat{\Omega}^{u}(\xi), X_{1}\right)<\delta / 4
$$

for $\xi \leq \xi_{L}\left(d_{L}\right)$ and for all sufficiently small $\varepsilon$. However by (6.5) and Gronwall's inequality it follows that

$$
\rho\left(\hat{e}_{1}\left(\xi_{L}, \lambda, \varepsilon_{n}\right), \hat{e}_{*}\left(\xi_{L}\right)\right)<\delta / 4
$$

for sufficiently small $\varepsilon$. Since $\hat{e}_{1} \in \widehat{\Omega}^{u}(\xi)$ the previous two inequalities contra$\operatorname{dict}(6.6)$.

We have now established that $\hat{e}_{*} \in X_{1}$. Since there is a unique solution of the projectivized reduced system (4.22) which tends to $\hat{e}_{1 R C}^{-}(\lambda)$ as $\xi \rightarrow-\infty$, namely $\hat{e}_{1 R}(\xi, \lambda)$, it suffices to show that $\hat{e}_{1 *}(\xi)$ also satisfies this condition. Since $\hat{e}_{*}(\xi) \in X_{1}$ for all $\xi$ the only alternative would be that $\hat{e}_{*}(\xi)$ tends to $e_{4 R C}^{-}(\lambda)$ as $\xi \rightarrow-\infty$. This possibility has already been eliminated above. 
We have proved that $\mathscr{E}_{1}(\varepsilon)$ and $\mathscr{E}_{1 R}$ are uniformly close in the interior of $[-1,1] \times K$. We next introduce a family of intermediate bundles $\mathscr{F}(\sigma, \varepsilon)$ depending on a pair of parameters $\sigma=\left(\sigma_{L}, \sigma_{R}\right)$, which will equal $\mathscr{E}_{1}(\varepsilon)$ on $\left[\sigma_{L}, \sigma_{R}\right] \times K$ but which will excise the portions of $\mathscr{E}_{1}(\varepsilon)$ over the slow manifolds. Let $\sigma$ be chosen so that

$$
-1 \leq \sigma_{L}<0<\sigma_{R} \leq 1
$$

The base space $B_{\sigma}$ of $\mathscr{F}(\sigma, \varepsilon)$ is

$$
B_{\sigma}=b_{L} \cup b_{0} \cup b_{R}
$$

where

$$
\begin{aligned}
& b_{L}=\left\{\sigma_{L}\right\} \times K^{0} \quad \text { (left cap), } \\
& b_{0}=\left[\sigma_{L}, \sigma_{R}\right] \times K \text { (sides), } \\
& b_{R}=\left\{\sigma_{R}\right\} \times K^{0} \quad \text { (right cap). }
\end{aligned}
$$

The bundle $\mathscr{F}(\sigma, \varepsilon)$ will be defined as the pullback $\hat{f}^{*} \Gamma_{1}\left(\mathbb{C}^{4}\right)$ of the universal bundle $\Gamma_{1}\left(\mathbb{C}^{4}\right)$ over $\mathbb{C} P^{3}$ by a certain map

$$
\hat{f}_{\sigma, \varepsilon}=\hat{f}: B_{\sigma} \rightarrow \mathbb{C} P^{3}
$$

which is defined below.

First we need to specify the appropriate ranges for $\sigma_{L}$ and $\sigma_{R}$. Select values $\xi_{L}(\varepsilon), \xi_{R}(\varepsilon)$ so that

$$
\begin{aligned}
& \left|x\left(\xi_{L}(\varepsilon), \varepsilon\right)-x_{L}\right| \rightarrow 0 \quad \text { as } \varepsilon \rightarrow 0, \\
& \left|x\left(\xi_{R}(\varepsilon), \varepsilon\right)-x_{R}\right| \rightarrow 0 \quad \text { as } \varepsilon \rightarrow 0,
\end{aligned}
$$

where $x_{L}$ and $x_{R}$ are the left and right corners of the singular front. Let $\sigma_{L}(\varepsilon)$ and $\sigma_{R}(\varepsilon)$ denote the values of $\tau$ associated with $\xi_{L}(\varepsilon)$ and $\xi_{R}(\varepsilon)$. We suppose in addition to (6.7) that $\sigma_{L}$ and $\sigma_{R}$ are selected so that

$$
\sigma_{L} \leq \sigma_{L}(\varepsilon), \quad \sigma_{R}(\varepsilon) \leq \sigma_{R} .
$$

We remark that it follows from (6.8) that

$$
\sigma_{L}(\varepsilon) \rightarrow-1 \quad \text { and } \quad \sigma_{R}(\varepsilon) \rightarrow+1 \quad \text { as } \varepsilon \rightarrow 0 .
$$

We can now define the map $\hat{f}_{\sigma, \varepsilon}$. Let $\hat{e}_{1}(\tau, \lambda, \varepsilon)$ denote the fast unstable bundle in the present scaling for $\tau$; define

$$
\hat{f}_{\sigma, \varepsilon}(\tau, \lambda)=\hat{e}_{1}(\tau, \lambda, \varepsilon) \text { for }(\tau, \lambda) \in b_{L} \cup b_{0} .
$$

Since we cannot control $\hat{e}_{1}$ as $\tau \rightarrow+1$ for $\lambda \in K^{0}$ we define $\hat{f}_{\sigma, \varepsilon}$ on the right cap $b_{R}$ differently. Note that for $\tau \geq \sigma_{R}(\varepsilon)$ the underlying wave is near the slow manifold $S_{R}$ and, so, the fast unstable eigenvector $f_{1}(\tau, \lambda, \varepsilon)$ of the frozen matrix $a(\tau, \lambda, \varepsilon)$ is well defined. Furthermore, given a choice $e_{1}^{+}(\lambda, \varepsilon)$ of the fast unstable eigenvector at $\tau=+1, f_{1}$ may be selected so that

$$
\lim _{\tau \rightarrow+1} f_{1}(\tau, \lambda, \varepsilon)=e_{1}^{+}(\lambda, \varepsilon) \quad\left(\lambda \in K \cup K^{0}\right) .
$$




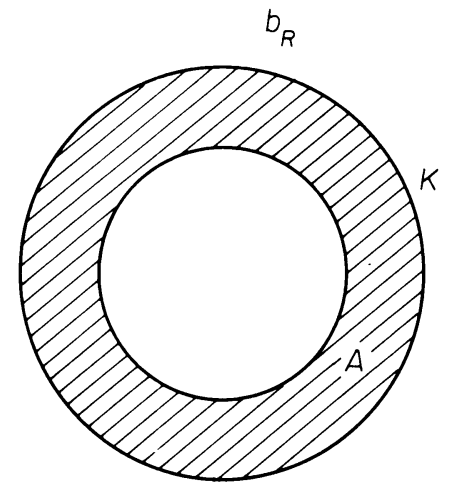

Figure 6.1

By the elephant trunk lemma, $\hat{e}_{1}(\tau, \lambda, \varepsilon)$ is uniformly approximated by $\hat{f}_{1}(\tau, \lambda, \varepsilon)$ for $\tau \geq \sigma_{R}(\varepsilon)$ and for $\lambda \in K$. For $\sigma_{R} \geq \sigma_{R}(\varepsilon)$ we can therefore select a representative $e_{1}\left(\sigma_{R}, \lambda, \varepsilon\right)$ in the fiber over $\hat{e}_{1}\left(\sigma_{R}, \lambda, \varepsilon\right)$ such that $e_{1}$ and $f_{1}$ are uniformly close for all $\lambda \in K$. Select a thin annular region $A \subset b_{R}$ as depicted in the figure, and let $\varphi(\lambda), \psi(\lambda)$ be a partition of unity in $b_{R}$ such that $\varphi \equiv 1$ near $K$ and $\varphi \equiv 0$ in $K^{0} \backslash A$. By the previous remarks it follows that for $\tau \geq \sigma_{R}(\varepsilon)$, the vector

$$
f_{\sigma, \varepsilon}\left(\sigma_{R}, \lambda\right)=\varphi(\lambda) e_{1}\left(\sigma_{R}, \lambda, \varepsilon\right)+\psi(\lambda) f_{2}\left(\sigma_{R}, \lambda, \varepsilon\right)
$$

is nonvanishing for all $\lambda \in K \cup K^{0}$ and matches up continuously with $e_{1}$ along $K$. We can therefore define $\hat{f}_{\sigma, \varepsilon}\left(\sigma_{R}, \varepsilon\right)$ to be the image of the above vector in $\mathbb{C} P^{3}$, to complete the definition of $\hat{f}_{\sigma, \varepsilon}$.

We can now show for sufficiently small but fixed $\varepsilon>0$ that each bundle $\mathscr{F}(\sigma, \varepsilon)$ associated with $\hat{f}_{\sigma, \varepsilon}$ is equivalent to $\mathscr{E}_{1}(\varepsilon)$ for all $\sigma$ satisfying (6.7) and (6.9). Let $h_{\sigma}: B \rightarrow B_{\sigma}$ be a homeomorphism mapping the sides, left cap, and right cap of $B$ onto the corresponding subsets of $B_{\sigma}$. We can clearly arrange that $h_{\sigma}$ is the identity when $\sigma=(-1,1)$. It suffices to show that the maps $f_{\sigma, \varepsilon} \circ h_{\sigma}: B \rightarrow \mathbb{C} P^{3}$ are all homotopy equivalent to $\hat{e}_{1}$. This follows immediately from (6.11) and the manner in which $\hat{f}_{\sigma, \varepsilon}$ was defined over the right cap, $b_{R}$. The family $\left\{\hat{f}_{\sigma, \varepsilon}\right\}$ is clearly the desired homotopy as $\sigma$ varies from $\left(\sigma_{L}, \sigma_{R}\right)$ to $(-1,1)$.

Now let $\sigma(\varepsilon)=\left(\sigma_{L}(\varepsilon), \sigma_{R}(\varepsilon)\right), \hat{f}_{\varepsilon}=\hat{f}_{\sigma(\varepsilon), \varepsilon}$, and $\mathscr{F}(\varepsilon)=\mathscr{F}(\sigma(\varepsilon), \varepsilon)$; we have shown for all sufficiently small but fixed $\varepsilon>0$ that $\mathscr{F}(\varepsilon)$ is equivalent to $\mathscr{E}_{1}(\varepsilon)$. We finally claim that $\mathscr{F}(\varepsilon)$ is equivalent to $\mathscr{E}_{1 R}$. If $B_{\varepsilon}=B_{\sigma(\varepsilon)}$ and $h_{\varepsilon}=h_{\sigma(\varepsilon)}$ is the homeomorphism of $B$ onto $B_{\varepsilon}$ described above, it suffices to show that

$$
\lim _{\varepsilon \rightarrow 0}\left(\hat{f}_{\varepsilon} \circ h_{\varepsilon}(b)\right)=\hat{e}_{1 R}(b)
$$

uniformly for $b \in B$. To this end, let $\rho$ be a metric on $\mathbb{C} P^{3}$ and let $\delta>0$ 
be given. By (6.8), (6.11), and the elephant trunk lemma it follows that there exist $d_{L}, d_{R}, \eta_{L}, \eta_{R}$ depending on $\delta$, and $\tau_{L}, \tau_{R}$ associated with $\xi_{L}\left(d_{L}\right)$ and $\xi_{R}\left(d_{R}\right)$ such that

$$
\begin{array}{ll}
\rho\left(\widehat{\Omega}^{u}(\tau), \hat{e}_{1 R C}^{-}(\lambda)\right)<\delta & \text { for }(\tau, \lambda) \in\left[\sigma_{L}(\varepsilon), \tau_{L}\right] \times K, \\
\rho\left(\widehat{\Omega}^{u}(\tau), \hat{e}_{1 R C}^{+}(\lambda)\right)<\delta & \text { for }(\tau, \lambda) \in\left[\tau_{R}, \sigma_{R}(\varepsilon)\right] \times K ;
\end{array}
$$

recall that $\hat{e}_{1 R C}^{ \pm}(\lambda)$ are the limits of $\hat{e}_{1 R}(\tau, \lambda)$ as $\tau \rightarrow \pm 1$. This sets the value of $\tau_{L}$ and $\tau_{R}$ independently of $\varepsilon$. Since $\hat{f}_{1}$ and $\hat{e}_{1}$ both lie in $\hat{\Omega}^{u}(\tau)$ for $\tau \leq \tau_{L}$ and $\tau \geq \tau_{R}$ it follows that there exist $\tau_{1} \leq \tau_{L}$ and $\tau_{2} \geq \tau_{R}$ such that

$$
\rho\left(\hat{f}_{\varepsilon} \circ h_{\varepsilon}(\tau, \lambda), \hat{e}_{1 R}(\tau, \lambda)\right)<\delta
$$

for $\tau \leq \tau_{1}$ or $\tau \geq \tau_{2}$ and all appropriate $\lambda$. Here $\tau_{1}$ and $\tau_{2}$ depend only on $\tau_{L}$ and $\tau_{R}$ and the rate of convergence of $\hat{e}_{1 R}(\tau, \lambda)$ to its limits at $\tau= \pm 1$.

With $\tau_{1}$ and $\tau_{2}$ now fixed as above we can apply Lemma 6.3 to conclude that

$$
\rho\left(\hat{f}_{\varepsilon} \circ h_{\varepsilon}(b), \hat{e}_{1 R}(b)\right) \leq \delta
$$

for $b \in B \cap\left\{\tau_{1} \leq \tau \leq \tau_{2}\right\}$ for all sufficiently small $\varepsilon$.

We summarize the above result in the following proposition.

Proposition 6.4. The bundle $\mathscr{E}_{1}(\varepsilon)$ is equivalent to $\mathscr{E}_{1 R}$, and

$$
c_{1}\left(\mathscr{E}_{1}(\varepsilon)\right)=c_{1}\left(\mathscr{E}_{1 R}\right)
$$

for all sufficiently small $\varepsilon>0$.

D. Continuation of $\mathscr{E}_{2}(\varepsilon)$ to $\mathscr{E}_{2 R}$. By Theorem $5.3, \mathscr{E}_{2}(\varepsilon)$ is closely approximated by $\mathscr{E}_{2 R}$ away from the transition layer which, in the $T$-scaling in (6.4), coincides with the complement of an interval of $\mathscr{O}(\varepsilon)$ about $T=0$ in $[-1,1]$. However, the bundles will significantly differ in the transition. The homotopy of $\mathscr{E}_{2}(\varepsilon)$ to $\mathscr{E}_{2 R}$ proceeds in three steps. First we regard $\varepsilon>0$ as small but fixed and introduce a family of bundles $\mathscr{E}_{2}(S, \varepsilon)$ which depend continuously on parameters $S=\left(S_{L}, \sigma\right)$. Fix $S_{L}, S_{R}$ satisfying

$$
-1<S_{L}<0<S_{R}<+1 \text {; }
$$

the parameter $\sigma$ assumes values in $\left[S_{L}, S_{R}\right] . \mathscr{E}_{2}(S, \varepsilon)$ will consist of the restriction of $\mathscr{E}_{2}(\varepsilon)$ to the complement of $\left[S_{L}, \sigma\right]$ in $[-1,1]$, with the two faces $T=S_{L}, T=\sigma$ glued together in a suitable manner.

More precisely, let the base space of $\mathscr{E}_{2}(S, \varepsilon)$ be a sphere $B_{S}$ obtained by gluing the hemispheres

$$
b_{-}\left(S_{L}\right)=B \cap\left\{T \leq S_{L}\right\}, \quad b_{+}(\sigma)=B \cap\{T \geq \sigma\}
$$

together along their boundaries. After the identification has been performed, $b_{-}\left(S_{L}\right)$ and $b_{+}(\sigma)$ intersect along a curve $\widehat{K}$, which is a copy of $K$ in $B_{S}$. Let

$$
\mathscr{E}_{2}^{-}\left(S_{L}, \varepsilon\right)=\mathscr{E}_{2}(\varepsilon)\left|b_{-}\left(S_{L}\right), \quad \mathscr{E}_{2}^{+}(\sigma, \varepsilon)=\mathscr{E}_{2}(\varepsilon)\right| b_{+}(\sigma) .
$$


To complete the construction we define a gluing map $\varphi_{S, \varepsilon}$,

$$
\varphi_{S, \varepsilon}: \mathscr{E}_{2}^{-}\left(S_{L}, \varepsilon\right) \mid \widehat{K} \rightarrow \mathscr{E}_{2}^{+}(\sigma, \varepsilon) .
$$

The flow associated with (3.3) provides a natural way to construct such an isomorphism. Let $\zeta_{L}$ and $\zeta_{\sigma}$ be the values of $\zeta$ respectively associated with $T=S_{L}$ and $\sigma$, in (6.4). Let $E_{2}(\zeta, \lambda, \varepsilon)$ be the solution of $(3.3)_{s}$ with data $E_{L}$ at $\zeta=\zeta_{L}$, where $E_{L}$ lies in the fiber of $\mathscr{E}_{2}^{-}\left(S_{L}, \varepsilon\right)$ over $\left(S_{L}, \lambda\right)$, and define

$$
\varphi_{S, \varepsilon} E_{L}=E_{2}\left(\zeta_{\sigma}, \lambda, \varepsilon\right)
$$

The bundle $\mathscr{E}_{2}(S, \varepsilon)$ is then defined to be

$$
\mathscr{E}_{2}(S, \varepsilon)=\mathscr{E}_{2}^{-}\left(S_{L}, \varepsilon\right) \cup_{\varphi_{S, \varepsilon}} \mathscr{E}_{2}^{+}(\sigma, \varepsilon) .
$$

When $\sigma=S_{L}, \varphi_{\sigma, \varepsilon}$ is the identity so that

$$
\mathscr{E}_{2}\left(S_{L}, S_{L}, \varepsilon\right) \cong \mathscr{E}_{2}(\varepsilon) .
$$

Now increase $\sigma$ from $S_{L}$ to $S_{R}$. Since the flow map is continuous for $\varepsilon>0$ it follows that $\varphi_{S, \varepsilon}$ form a homotopy of isomorphisms. Thus if $S=\left(S_{L}, S_{R}\right)$ it follows from Lemma 6.2 and the above that

$$
\mathscr{E}_{2}(\varepsilon) \cong \mathscr{E}_{2}(S, \varepsilon) \text {. }
$$

The next step of the homotopy consists of fixing $S=\left(S_{L}, S_{R}\right)$ as in (6.12) and allowing $\varepsilon$ to tend to zero. By (a) and (c) of Theorem 5.3 it follows that

$$
\lim _{\varepsilon \rightarrow 0} \widehat{E}_{2}(T, \lambda, \varepsilon)=\widehat{E}_{2 R}(T, \lambda)
$$

uniformly for $(T, \lambda) \in b_{-}\left(S_{L}\right) \cup b_{+}\left(S_{R}\right)$. It also follows that the gluing maps $\varphi_{S, \varepsilon}$ tend to a limit $\varphi_{S, 0}$ as $\varepsilon \rightarrow 0$ which is explicitly determined as follows. Let $\zeta_{L}, \zeta_{R}$ be the values of $\zeta$ associated with $S_{L}, S_{R}$ and let $Z_{R}(\zeta, \lambda)$ be a solution of $(5.3)_{R}$ such that

$$
\widehat{E}_{2 R}(\zeta, \lambda)=\left[i_{R}(\zeta, \lambda) Z_{R}(\zeta, \lambda)\right]^{\curvearrowright} .
$$

By (a) of Theorem 5.3 it follows that

$$
\varphi_{S, 0}\left[i_{R}\left(S_{L}, \lambda\right) Z_{R}\left(S_{L}, \lambda\right)\right]=i_{R}\left(\zeta_{R}, \lambda\right) Z_{R}\left(\zeta_{R}, \lambda\right) .
$$

Define $\mathscr{E}_{2}(S, 0)$ by

$$
\mathscr{E}_{2}(S, 0)=\mathscr{E}_{2 R} \mid b_{-}\left(S_{L}\right) \cup_{\varphi_{S_{0}}} b_{+}\left(S_{R}\right)
$$

it follows from the above that $\mathscr{E}_{2}(\varepsilon) \cong \mathscr{E}_{2}(S, 0)$ for each $S=\left(S_{L}, S_{R}\right)$ satisfying (6.12).

The final step is to allow $S_{L}$ to approach zero from below and $S_{R}$ to approach zero from above in $\mathscr{E}_{2}(S, 0)$. By (b) of Theorem 5.3 it follows that $\varphi_{S, 0}$ approaches the glueing map $\varphi_{R}$ used in the definition of $\mathscr{E}_{2 R}$.

We summarize the above results in the following proposition. 
Proposition 6.5. For all sufficiently small $\varepsilon>0, \mathscr{E}_{2}(\varepsilon)$ is equivalent to $\mathscr{E}_{2 R}$ and

$$
c_{1}\left(\mathscr{E}_{2}(\varepsilon)\right)=c_{1}\left(\mathscr{E}_{2 R}\right)=c_{1}\left(\tilde{\mathscr{E}}_{2 R}\right),
$$

where $\tilde{\mathscr{E}}_{2 R}$ is the bundle obtained from $\mathscr{E}_{2 R}$ by projection onto the 2-components.

E. The Chern number computation. The proof of the Stability Theorem will be completed by establishing the following lemma.

Lemma 6.6. $c_{1}(\mathscr{E}(\varepsilon))$ for all sufficiently small $\varepsilon>0$.

Proof. By (6.1) and Propositions 6.4 and 6.5 it suffices to show that the following are true:

(1) $c_{1}\left(\mathscr{E}_{1 R}\right)=1$

(2) $c_{1}\left(\tilde{\mathscr{E}}_{2 R}\right)=0$.

Proof of (1). The bundle $\mathscr{E}_{1 R}$ is constructed from $\left(p_{1 R}(\xi), q_{1 R}(\xi), 0,0\right)^{t}$, the solutions of $(3.9)_{f R}$ whose 2-components vanish identically. The equations (4.17) satisfied by the 1-components are the linearization about the monotone travelling wave solution of the bistable equation

$$
u_{1_{t}}=u_{1_{x x}}+u_{1} f_{1}\left(u_{1}, \alpha\right)
$$

which connects $u_{1}=0$ to $u_{1}=p(\alpha)$. It is known that such waves are linearly and hence, nonlinearly stable. In particular, it follows from the theorem of Fife and McLeod (see [FM]) that (4.17) admits precisely one eigenvalue in $K$ which occurs at $\lambda=0$; it is also proved in [FM] that $\lambda=0$ is simple. By theorem in [AGJ] it follows that $c_{1}\left(\mathscr{E}_{1 R}\right)$ is the number of eigenvalues of (4.17) inside $K$ including algebraic multiplicity which, together with [FM], implies (1).

Proof of $(2)$. Since $\tilde{\mathscr{E}}_{2 R}$ is the bundle obtained by projection onto the 2-components of the fibers of $\mathscr{E}_{2 R}$, by the main theorem in [AGJ] it suffices to show that $\lambda$ is not an eigenvalue of (3.9) $)_{s R}$ for all $\lambda \in K \cup K^{0}$. This is equivalent to showing that equations $(5.3)_{R}$ for the 2-components do not admit a uniformly bounded solution, $Z_{R}(\zeta, \lambda)$, for all such $\lambda$. In components these equations are

$$
\dot{P}_{2 R}=Q_{2 R}, \quad \dot{Q}_{2 R}=C_{R}(\zeta, \lambda) P_{2 R},
$$

where

$$
C_{R}(\zeta, \lambda)=\lambda-A_{22}^{R}(\zeta)-\frac{A_{12}^{R}(\zeta) A_{21}^{R}(\zeta)}{\lambda-A_{11}^{R}(\zeta)} .
$$

Recall that $\left(A_{i j}^{R}(\zeta)\right)$ is the Jacobian matrix of $\left(u_{1} f_{1}, u_{2} f_{2}\right)$ evaluated at the (discontinuous) slow singular limit $X_{R}(\zeta)$. In particular, $U_{1 R}(\zeta) \equiv 0$ for $\zeta<0$ and $U_{1 R}(\zeta)=p\left(U_{2 R}(\zeta)\right)$ for $\zeta>0$. From the hypotheses in $\S$ IIA and the above, it follows that $A_{12}^{R}(\zeta) A_{21}^{R}(\zeta)$ is strictly negative for $\zeta>0$ and vanishes identically for $\zeta<0$. It should also be noted that $A_{22}^{R}(\zeta)$ is negative at $\zeta=$ $\pm \infty$; however this term may change signs along the right branch of the slow manifold $u_{1}=p\left(u_{2}\right)$. 
It will be convenient to consider the projectivized variable $\hat{Z}_{R}(\zeta, \lambda)$ associated with a solution $Z_{R}(\zeta, \lambda)$ of (6.13). The equations for $\widehat{Z}_{R},(5.5)_{R}$, can be expressed in terms of the local coordinate $S_{R}=Q_{2 R} / P_{2 R}$, which satisfies

$$
\dot{S}_{R}=C_{R}(\zeta, \lambda)-S_{R}^{2} \text {. }
$$

The roots of the asymptotic systems for (6.14) are

$$
r_{1}^{ \pm}(\lambda)=\sqrt{C_{R}( \pm \infty, \lambda)}, \quad r_{2}^{ \pm}(\lambda)=-\sqrt{C_{R}( \pm \infty, \lambda)} ;
$$

we take the standard branch of the square root so that $\operatorname{Re} \sqrt{z}>0$ if $\operatorname{Re} z>0$. It follows that $r_{1}^{ \pm}(\lambda)$ are attractors and $r_{2}^{ \pm}(\lambda)$ are repellers for the asymptotic systems associated with (6.14).

In order to show that $\lambda \in K \cup K^{0}$ is not an eigenvalue of (3.9) $)_{s R}$ it suffices to show that the (uniquely determined) solution $S_{R}(\zeta, \lambda)$ which tends to $r_{1}^{-}(\lambda)$ as $\zeta \rightarrow \infty$ does not tend to $r_{2}^{+}(\lambda)$ as $\zeta \rightarrow+\infty$, since the latter condition implies that every solution of $(6.13)_{R}$ which decays to zero as $\zeta \rightarrow-\infty$ becomes unbounded as $\zeta \rightarrow+\infty$.

Since $S_{R}$ is a complex variable it will be convenient to write (6.14) in real and imaginary parts. To this end let

$$
\begin{gathered}
\lambda=\alpha+i \beta, \\
C_{R}(\zeta, \lambda)=g(\zeta, \lambda)+i h(\zeta, \lambda), \\
S_{R}(\zeta, \lambda)=\sigma(\zeta, \lambda)+i \tau(\zeta, \lambda),
\end{gathered}
$$

so that $(6.14)$ is equivalent to

$$
\dot{\sigma}=g+\tau^{2}-\sigma^{2}, \quad \dot{\tau}=h-2 \sigma \tau .
$$

Here, $g$ and $h$ are given explicitly by the expressions

$$
\begin{gathered}
g(\zeta, \lambda)=\alpha-A_{22}^{R}(\zeta)-\frac{A_{12}^{R}(\zeta) A_{21}^{R}(\zeta)}{\left(\alpha-A_{11}^{R}(\zeta)\right)^{2}+\beta^{2}}\left(\alpha-A_{11}^{R}(\zeta)\right), \\
h(\zeta, \lambda)=\left[1+\frac{A_{12}^{R}(\zeta) A_{21}^{R}(\zeta)}{\left(\alpha-A_{11}^{R}(\zeta)\right)^{2}+\beta^{2}}\right] \beta .
\end{gathered}
$$

We shall treat the case of real $\lambda$ and complex $\lambda$ separately.

Case 1: $\beta \neq 0$. Without loss of generality we can assume that $\beta>0$. By hypothesis $(\mathrm{H} 2)$ in the statement of the Stability Theorem it then follows that $h(\zeta, \lambda)>0$ for all $\zeta$. In particular, we have for such $\lambda$ that

$$
\operatorname{Im} r_{1}^{ \pm}(\lambda)>0, \quad \operatorname{Im} r_{2}^{ \pm}(\lambda)<0 .
$$

From the second equation in (6.15) and the positivity of $h$ it is immediate that the half-plane $\{(\sigma, \tau): \tau \geq 0\}$ is positively invariant for continuous solutions of (6.15). If the solution $S_{R}(\zeta, \lambda)$ remains uniformly bounded for all $\zeta$ it follows that it cannot tend to $r_{2}^{+}(\lambda)$ as $\zeta \rightarrow+\infty$; indeed it must then tend to $r_{1}^{+}(\lambda)$. 
It may occur that $S_{R}(\zeta, \lambda)$ blows up in finite or infinite time. In this case we must return to the coordinate $\widehat{Z}_{R}$ on the Riemann sphere, $\mathbb{C} P^{1}$. Viewing $\mathbb{C} P^{1}$ as a sphere over the $(\sigma, \tau)$ plane with south pole at the origin, the point at infinity corresponds to the north pole, and the closure $H$ of the image of the half-plane $\{\tau \geq 0\}$ is a hemisphere with boundary passing through the north pole. If $S_{R}(\zeta, \lambda)$ blows up in infinite time, then its $\omega$ limit set lies in $H$ and it must therefore be different from $r_{2}^{+}(\lambda)$.

Suppose then that $S_{R}(\zeta, \lambda)$ becomes unbounded as $\zeta \rightarrow \zeta_{0}$ for some $\left|\zeta_{0}\right|<$ $\infty$. Since $S_{R}(\zeta, \lambda)$ tends to $r_{1}^{-}(\lambda) \in H$ as $\zeta \rightarrow-\infty$ we can assume that $\zeta_{0}$ is the smallest value of $\zeta$ below which $S_{R}$ stays in $H$ and remains finite.

Suppose that $\widehat{Z}_{R}(\zeta, \lambda)$ exits $H$ for some $\zeta \geq \zeta_{0}$. A contradiction is obtained as follows. Let $T=S_{R}^{-1}$ so that $T(\zeta, \lambda)$ is well defined for all $\zeta$ near $\zeta_{0}$. Let $T=s+i t$; the equations for $s$ and $t$ are

$$
\dot{s}=1+g\left(t^{2}-s^{2}\right)+2 h s t, \quad \dot{t}=h\left(t^{2}-s^{2}\right)-2 g s t ;
$$

we also have that

$$
s=\frac{\sigma}{\sigma^{2}+\tau^{2}}, \quad t=\frac{-\tau}{\sigma^{2}+\tau^{2}} .
$$

By hypothesis, at least one of $\sigma, \tau$ blow up as $\zeta \rightarrow \zeta_{0}$; it follows from the above that both $s$ and $t$ tend to zero as $\zeta \rightarrow \zeta_{0}$. Furthermore, since $\hat{Z}_{R}(\zeta, \lambda) \in H$ for $\zeta \leq \zeta_{0}$ it follows that $t(\zeta)<0$ for $\zeta<\zeta_{0}$.

A contradiction is obtained by expanding $s(\zeta)$ and $t(\zeta)$ in Taylor series about $\zeta=\zeta_{0}$. From the first equation in (6.16) we have that

$$
s(\zeta)=\left(\zeta-\zeta_{0}\right)+\mathscr{O}\left(\zeta-\zeta_{0}\right)^{2} .
$$

From the second equation in (6.18) we have that $\dot{t}(0)=0$ and $\ddot{t}(0)=0$, since $s(0)=t(0)=0$. It then follows from the expansion for $s(\zeta)$ that

$$
t(0)=-2 h\left(\zeta_{0}, \lambda\right),
$$

so that

$$
t(\zeta)=-\frac{2}{3} h\left(\zeta_{0}, \lambda\right)\left(\zeta-\zeta_{0}\right)^{3}+\mathscr{O}\left(\zeta-\zeta_{0}\right)^{4}
$$

for $\zeta$ near $\zeta_{0}$. This contradicts $t(\zeta)<0$ for $\zeta<\zeta_{0}$.

We remark that if $\zeta_{0}=0$ then $h(\zeta, \lambda)$ is discontinuous at $\zeta_{0}$. However, the above $h$ and its derivatives are well behaved as $\zeta \rightarrow 0^{-}$; we can therefore apply the previous argument, which only depends on the values of $s$ and $t$ for $\zeta \leq \zeta_{0}$, by replacing $h$ with a function $H(\zeta, \lambda)$ extends $h$ for $\zeta<0$ smoothly across $\zeta=0$. A contradiction is still obtained from $\zeta<0$ as before.

Case 2: $\beta=0$. In this case $h \equiv 0$ and from the second equation in (6.15) it follows that the solution which tends to $r_{1}^{-}(\lambda)$ as $\zeta \rightarrow-\infty$ must have $\tau \equiv 0$. The equation for $\sigma$ is therefore

$$
\dot{\sigma}=g(\zeta, \alpha)-\sigma^{2},
$$


where

$$
g(\zeta, \alpha)=\alpha-A_{22}^{R}(\zeta)-\frac{A_{12}^{R}(\zeta) A_{21}^{R}(\zeta)}{\alpha-A_{11}^{R}(\zeta)} .
$$

We begin by noting that if $\sigma(\zeta, \lambda)$ is the solution of $(6.17)$ which tends to $r_{1}^{-}(\lambda)$ as $\zeta \rightarrow-\infty$ then

$$
\sigma(\zeta, \alpha) \leq \sigma\left(\zeta, \alpha_{1}\right) \quad(\zeta \in \mathbb{R})
$$

whenever $\alpha \leq \alpha_{1}$. This follows from the observation that for $\alpha \geq 0$,

$$
\frac{\partial}{\partial \alpha} g(\zeta, \lambda)=1+\frac{A_{12}^{R}(\zeta) A_{21}^{R}(\zeta)}{\left(\lambda-A_{11}^{R}(\zeta)\right)^{2}} \geq 1+\frac{A_{12}^{R}(\zeta) A_{21}^{R}(\zeta)}{A_{11}^{R}(\zeta)^{2}}>0
$$

where the last inequality is hypothesis (H2). It follows that this inequality holds for $\alpha \geq-\delta$ for sufficiently small $\delta>0$. We can always arrange for this through a suitable choice of the curve $K$. This inequality, which is valid for $-\infty \leq \zeta \leq+\infty$ also implies

$$
\frac{\partial}{\partial \alpha} r_{1}^{-}(\lambda)>0
$$

for $\alpha \geq-\delta$. The latter condition implies (6.18) for $\zeta$ sufficiently negative; (6.18) then follows from (6.19) for all $\zeta$ by a standard comparison theorem.

The proof will be complete if it can be shown that

$$
\lim _{\zeta \rightarrow+\infty} \sigma(\zeta, 0)=r_{1}^{+}(0)
$$

It will be convenient to return to the original equations (6.13). Let $g_{ \pm}\left(U_{2}\right)$ be the nonlinear functions in the slow limit equations $(2.3)_{s R}^{ \pm}$. It follows that

$$
C_{R}(\zeta, 0)= \begin{cases}g_{+}^{\prime}\left(U_{2 R}(\zeta)\right) & (\zeta>0), \\ g_{-}^{\prime}\left(U_{2 R}(\zeta)\right) & (\zeta<0),\end{cases}
$$

where $U_{2 R}(\zeta)$ is the $u_{2}$-component of the slow singular limit. It follows that (6.13) at $\alpha=0$ is the equation of variations about the slow singular limit.

If $\left(P_{2 R}(\zeta), Q_{2 R}(\zeta)\right)^{t}$ is a solution of (6.13) which decays at $\zeta=-\infty$ it follows that for $\zeta<0$

$$
\left(P_{2 R}(\zeta), Q_{2 R}(\zeta)\right)^{t}=w\left(\dot{U}_{2 R}(\zeta), \dot{V}_{2 R}(\zeta)\right)^{t},
$$

for some $w \in \mathbb{C}$, i.e., it is a scalar multiple of the derivative of the slow singular limit. Without loss of generality we may take $w=1$.

The graph of the slow limit $\left(U_{2 R}(\zeta), V_{2 R}(\zeta)\right)$ is depicted in Figure 6.2.

The solution $\left(P_{2 R}, Q_{2 R}\right)^{t}$ is the tangent vector to this curve for $\zeta<0$. Since the left and right limits of the tangent vector to $\left(U_{2 R}(\zeta), V_{2 R}(\zeta)\right)$ are transverse to one another at $\zeta=0$ (see Figure 6.2) it follows that $\left(P_{2 R}(\zeta), Q_{2 R}(\zeta)\right)^{t}$ is transverse to $\left(\dot{U}_{2 R}(\zeta), \dot{V}_{2 R}(\zeta)\right)^{t}$ for all $\zeta>0$, i.e., these are independent solutions of $(6.13)$ for $\zeta>0$.

The stable subspace of $(6.13)$ at $\zeta=+\infty$ corresponds to the critical point $r_{2}^{+}(0)$ of the projectivized equation $(6.17)$ at $\zeta=+\infty$. Since $r_{2}^{+}(0)$ is a repeller 


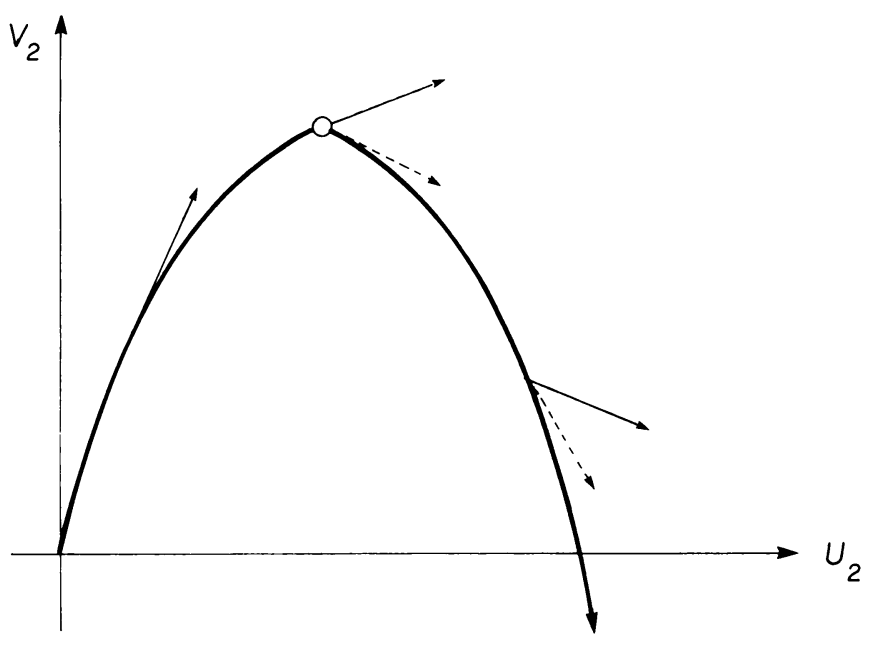

FIGURE 6.2

for $(6.17)$ at $\zeta=\infty$ it follows that there is a unique solution of $(6.17)$ which is asymptotic to $r_{2}^{+}(0)$ as $\zeta \rightarrow+\infty$. One such solution is given by

$$
\sigma_{2 R}(\zeta)=\dot{V}_{2 R}(\zeta) / \dot{U}_{2 R}(\zeta) \quad(\zeta>0)
$$

It easily follows that every other solution of (6.17) tends to $r_{1}^{+}(0)$ as $\zeta \rightarrow$ $+\infty$. Since $Q_{2 R}(\zeta) / P_{2 R}(\zeta)$ is distinct from $\sigma_{2 R}(s)$ it follows that $\left(P_{2 R}, Q_{2 R}\right)$ is unbounded as $\zeta \rightarrow+\infty$. Hence $\lambda \in K \cup K^{0}$ is not an eigenvalue of $(3.9)_{s R}$.

\section{REFERENCES}

[AGJ] J. Alexander, R. Gardner, and C. Jones, A topological invariant arising in the stability of travelling waves, (submitted).

[A] M. Atiyah, K-theory, Benjamin, New York, 1967.

[F] P. Fife, Mathematical aspects of reacting and diffusing systems, Lecture Notes in Biomath., vol. 28, Springer-Verlag, New York, 1979.

[FM] P. Fife and J. B. McLeod, The approach of solutions of nonlinear diffusion equations to travelling front solutions, Arch. Rational Mech. Anal. 65 (1977), 335-361.

[J] C. K. R. T. Jones, Stability of the travelling wave solution of the FitzHugh-Nagumo system, Trans. Amer. Math. Soc. 286 (1984), 431-469.

[G1] R. A. Gardner, Existence of travelling wave solutions of predator-prey systems via the connection index, SIAM J. Appl. Math. 44 (1984), 56-79.

[G2] _ Topological methods arising in the study of travelling waves, Reaction-Diffusion Equations (K. J. Brown and A. A. Lacey, eds.), Clarendon Press, Oxford, 1990, pp. 173-198.

[GS] R. A. Gardner and J. A. Smoller, The existence of periodic travelling waves for singularly perturbed predator-prey equations via the Conley index, J. Differential Equations (to appear).

[H] D. Henry, Geometric theory of semilinear parabolic equations, Lecture Notes in Math., vol. 840, Springer-Verlag, New York, 1981.

[K] T. Kato, Perturbation theory for linear operators, Springer-Verlag, Berlin, 1966.

[MS] J. W. Milnor and J. D. Stasheff, Characteristic classes, Ann. of Math. Studies, no. 76, Princeton Univ. Press, Princeton, N. J., 1974. 
[NF] Y. Nishiura and H. Fujii, Stability of singularly perturbed solutions to systems of reactiondiffusion equations, SIAM J. Math. Anal. 18 (1987), 1726-1770.

[NMIF] Y. Nishiura, M. Mimura, H. Ikeda, and H. Fujii, Singular limit analysis of stability of travelling wave solutions in bistable reaction-diffusion systems, preprint.

Department of Mathematics, University of Massachusetts, AmHerst, Massachusetts 01002

Department of Mathematics, University of Maryland, College Park, Maryland 20742

Current address: Division of Applied Mathematics, Brown University, Providence, R.I. 02912 